\title{
A Randomized Controlled Trial of Parent-Child Interaction Therapy with and without Incentives in a Community Mental Health Setting
}

Lauren Borduin Quetsch

Follow this and additional works at: https://researchrepository.wvu.edu/etd

\section{Recommended Citation}

Quetsch, Lauren Borduin, "A Randomized Controlled Trial of Parent-Child Interaction Therapy with and without Incentives in a Community Mental Health Setting" (2019). Graduate Theses, Dissertations, and Problem Reports. 6463.

https://researchrepository.wvu.edu/etd/6463

This Dissertation is protected by copyright and/or related rights. It has been brought to you by the The Research Repository @ WVU with permission from the rights-holder(s). You are free to use this Dissertation in any way that is permitted by the copyright and related rights legislation that applies to your use. For other uses you must obtain permission from the rights-holder(s) directly, unless additional rights are indicated by a Creative Commons license in the record and/ or on the work itself. This Dissertation has been accepted for inclusion in WVU Graduate Theses, Dissertations, and Problem Reports collection by an authorized administrator of The Research Repository @ WVU.

For more information, please contact researchrepository@mail.wvu.edu. 


\title{
A Randomized Controlled Trial of Parent-Child Interaction \\ Therapy with and without Incentives in a Community Mental \\ Health Setting
}

Lauren Borduin Quetsch, M.S.

\author{
Dissertation submitted \\ to the Eberly College of Arts and Sciences \\ at West Virginia University \\ in partial fulfillment of the requirements for the degree of \\ Doctor of Philosophy in \\ Psychology
}

Cheryl B. McNeil, Ph.D., Chair

Amy D. Herschell, Ph.D.

Elisa Krackow, Ph.D.

Aaron Metzger, Ph.D.

Lesley E. Cottrell, Ph.D.

Department of Psychology

Morgantown, West Virginia

2018

Keywords: attrition, homework, Parent-Child Interaction Therapy, treatment barriers, Hispanic

Copyright 2018 Lauren Borduin Quetsch 


\section{ABSTRACT \\ A Randomized Controlled Trial of Parent-Child Interaction Therapy with and without Incentives in a Community Mental Health Setting}

\section{Lauren Borduin Quetsch}

Disruptive behavior disorders (e.g., oppositional defiant disorder, conduct disorder) are the leading cause of referrals for children in mental health settings. Significant advancements in the implementation and dissemination of evidence-based family therapies have yielded successful reductions in these problem behaviors while also promoting long-term outcomes. Unfortunately, effective interventions such as Parent-Child Interaction Therapy (PCIT) are plagued with high rates of attrition and low rates of homework completion in community clinics. The current research study focused on evaluating the use of low cost incentives to increase retention and homework adherence in PCIT for a sample of low income, largely Hispanic families. Eighty-four families were randomly assigned to two groups: PCIT as usual or PCIT plus incentives. Findings indicated no significant differences between groups in rates of attendance, homework completion, or attainment of therapeutic skill mastery or graduation from treatment. Significant within-group outcomes were found for child behavior problems, internalizing and externalizing child behaviors, and parental stress, but no between-group differences were detected. A cost analysis between groups also was not significant. Exploratory analyses were conducted on factors contributing to early termination from treatment, therapy attitudes, and barriers to treatment participation. These analyses found that families who terminated from treatment early reported more difficulties with their children refusing to come to sessions, children developing new or different problems since starting treatment, and partners disagreeing with going to treatment. Additional analyses compared Hispanic and non-Hispanic families. Implications and future directions using innovative approaches to address attrition are discussed. 
Acknowledgements

This dissertation project would have been impossible without the boundless love, support, and flexibility of my incredible family. Tim, you have been my partner in every path I have pursued. Endless hours and discussions about my project make this accomplishment just as much yours as mine. To Layne and Little One, you provide meaning and balance to my life and I could not imagine my days without you. For my parents and brother, thank you for always believing in me and letting me achieve my goals without regret. For Tim's family, thank you for welcoming me as your daughter and supporting our wild adventures across the country in pursuit of my degree.

For my exceptional mentor, Dr. Cheryl McNeil. Thank you for providing numerous opportunities for me to become a better researcher, clinician, and grow into a more compassionate person. You have been a wonderful role model in work-life balance. I feel honored and truly lucky to have started my career under your boundless guidance. Your support in both my academic and life endeavors has allowed me to feel capable of handling any challenge that comes my way with a fearless determination.

Thank you as well, Dr. Amy Herschell, for allowing me to work in your lab, expanding my passions, and giving me reassurance in my career choices. You have always treated me as one of your own students and provided me the chance to be a stronger researcher in dissemination and implementation science. Your dedication to making a difference in the field inspires me to also make a meaningful impact in my work.

A special heartfelt thank you is dedicated to Dr. Emma Girard and the tireless Riverside team. First, Dr. Girard, your passion, organization, and energetic persona allowed this project to not only come together, but to be executed with an attention to detail a researcher could only ever dream about. You are the heart and soul behind this project and I could not fathom doing this study without you. In turn, thank you to the unequalled Riverside clinicians: Dinery C. Egan, Jaimee Rivera, Jennifer Dixon, Lisa-Marie Gonzalez, Starr Downey, Diana Jordan-Lloyd, Tanna Montecino, and Victoria E. Montes-Vu. Thank you for taking this journey with me and getting out of your comfort zone to test a new method for keeping our wonderful families enrolled in PCIT. And of course, Natacha Sanchez, truly thank you. Your upbeat attitude for handling thousands of pages of paperwork and data was the key to getting this project organized and completed on time. I am so happy for your promotion and wish you all the best.

Also, thank you to my lab: Corey Lieneman, April Highlander, Allison Thompson, Emma Veshecco, Karissa Bjorkgren, Cassandra Drain, Hannah Coffey, Kelsey Eackles, Maggie Ruckle, Morgan Simpson, Jana El-Khatib, and Nila Manivannan. This process of collecting and entering data would have been an insurmountable feat without you. To those friends and colleagues who supported me in graduate school and during this project, thank you. 
Table of Contents

List of Tables..................................................................

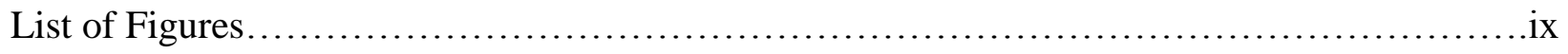

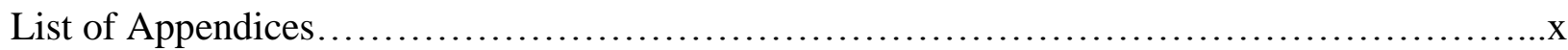

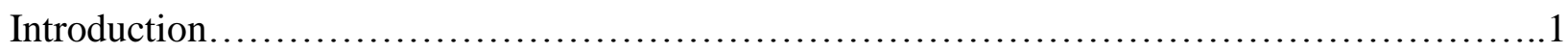

Attrition....................................................................

Homework ..............................................................

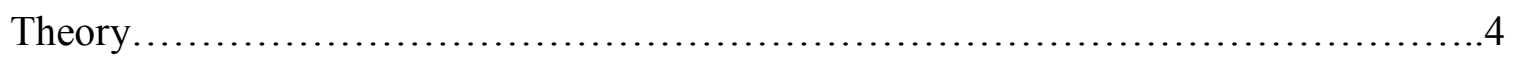

Parent-Child Interaction Therapy...........................................4

Attrition in PCIT ...........................................................

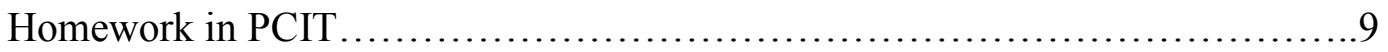

Incentives............................................................... 10

Incentives with Substance Abuse.........................................11

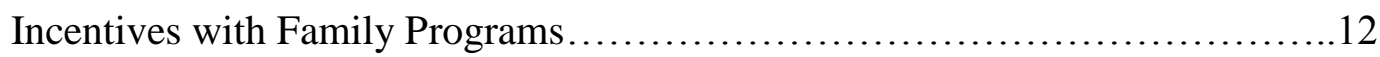

Concerns with Incentives........................................... 15

Cost of Incentives versus Attrition.................................... 17

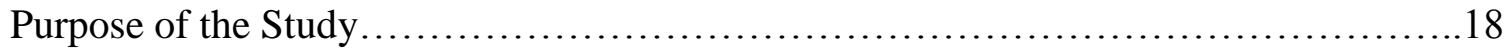

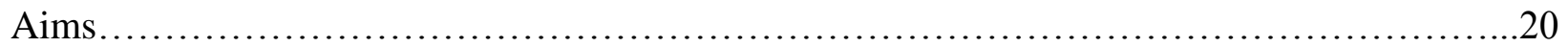

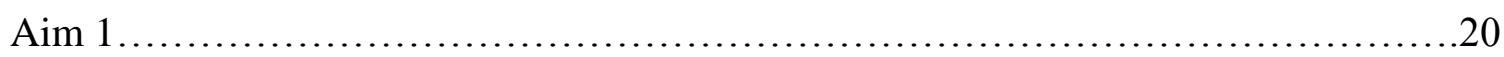

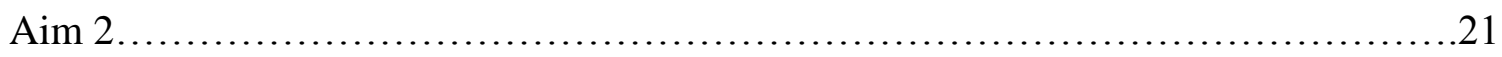

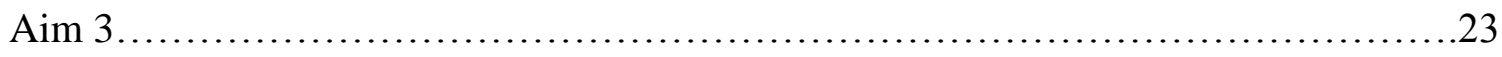

Exploratory Question....................................................23

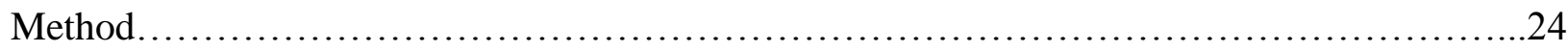

Participants...............................................................

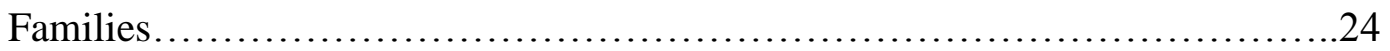




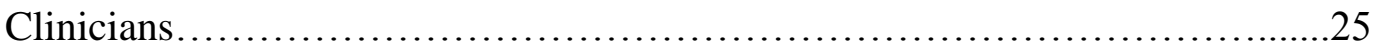

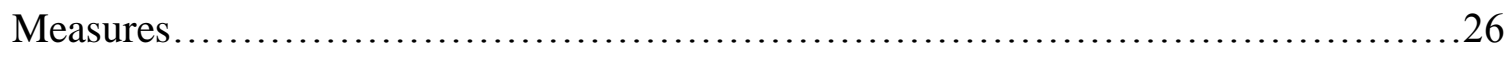

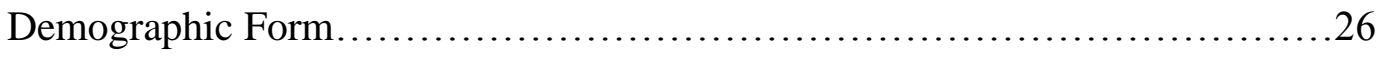

Barriers to Treatment Participation Scale.....................................27

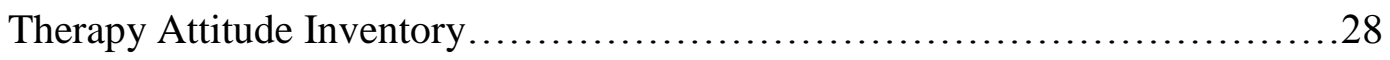

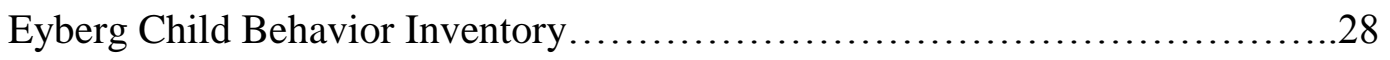

Child Behavior Checklist.....................................................29

Parenting Stress Index: Short Form.......................................30

Dyadic Parent-Child Interaction Coding System..............................31

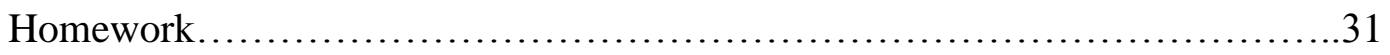

Session Record Form..................................................... 32

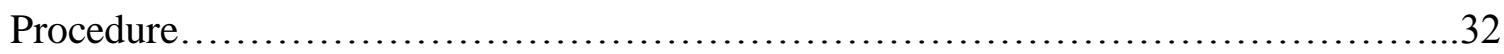

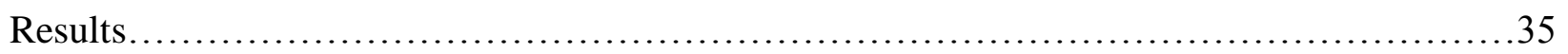

Power Analysis and Randomization.................................................35

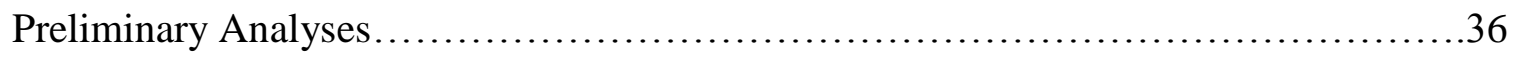

Demographic Information......................................................

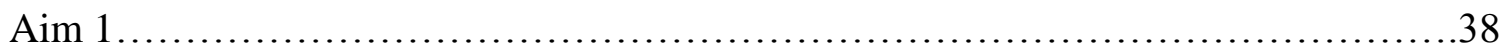

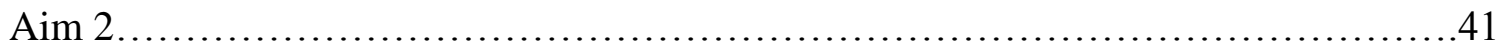

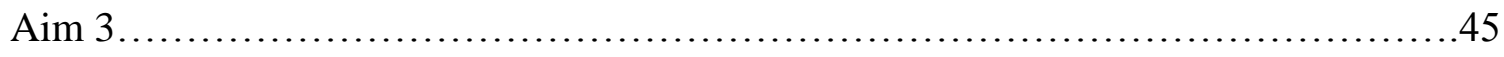

Exploratory Question............................................................ 47

Binary Logistic Regression...........................................47

TAI \& BTPS ............................................................... 48

Comparing the Current Sample to a Standardized Sample................48

General Outcomes................................................... 49

Outcomes by Incentive Group Status................................49 
Outcomes by CDI Mastery Status................................49

Outcomes by PCIT Graduation Status............................50

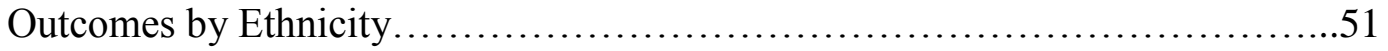

CDI Mastery and Graduation Status..................................51

Child Behavior Problems...........................................51

TAI \& BTPS ...................................................... 52

Discussion.................................................................... 52

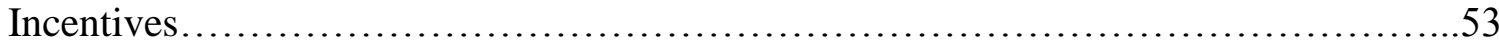

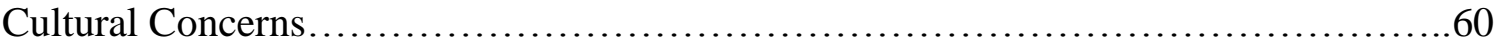

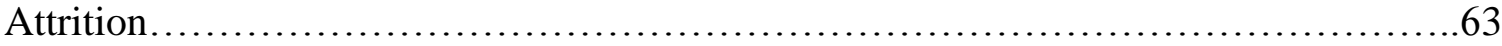

Rate of Homework Completion.............................................65

Cost Analysis.........................................................67

Barriers to Treatment/Predictors of Family Success.............................68

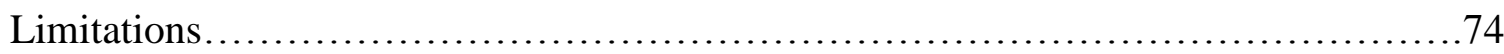

Clinical Implications and Future Research........................................ 77

References..................................................................... 81 


\section{List of Tables}

Table 1. Therapist Demographics.................................................... 104

Table 2a. Between-group Differences on Continuous Family Demographic Variables.........105

Table 2b. Between-group Differences on Categorical Family Demographic Variables:

Caregiver Role......................................................... 105

Table 2c. Between-group Differences on Categorical Family Demographic Variables:

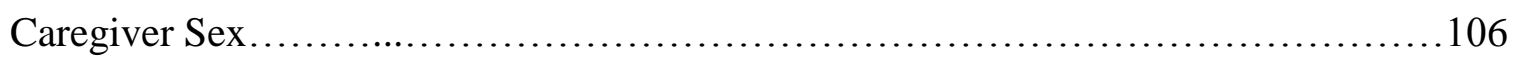

Table 2d. Between-group Differences on Categorical Family Demographic Variables:

Caregiver Ethnicity ...................................................... 106

Table 2e. Between-group Differences on Categorical Family Demographic Variables:

Caregiver Mental Health History........................................... 107

Table 2f. Between-group Differences on Categorical Family Demographic Variables:

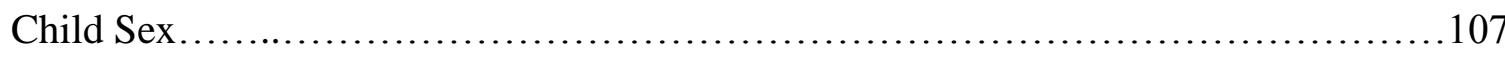

Table 2g. Between-group Differences on Categorical Family Demographic Variables:

Child Ethnicity......................................................... 108

Table 2h. Between-group Differences on Categorical Family Demographic Variables:

Child Mental Health History............................................... 108

Table 3. Between-group Differences in Number of Sessions and Length of Time to CDI

Mastery........................................................... 109

Table 4. Between-group Differences in Family Termination Prior to CDI Mastery............110

Table 5. Between-group Differences in Treatment Adherence Components...................111

Table 6. Between-group Differences in Family Attainment of CDI Mastery.................112

Table 7. Between-group Differences in Family Attainment of PDI Mastery/Graduation.......113

Table 8a. Between-group Mean Comparisons of Child Behavior Problems from First to Last

Attended Session - T-Scores............................................ 114

Table 8b. Between-group Mean Comparisons of Child Behavior Problems from First to Last

Attended Session - Raw Scores..........................................114 
Table 9. Between-group Mean Comparisons of Externalizing Child Behaviors throughout Treatment.................................................. 115

Table 10. Between-group Mean Comparisons of Internalizing Child Behaviors throughout Treatment.........................................................

Table 11. Between-group Mean Comparisons of Parenting Stress throughout Treatment......117

Table 12. Between-group Comparisons of Cost Analysis for the Community-Based Agency................................................................ 118

Table 13. Binary Logistic Regression for Exploratory Variables Contributing to Treatment

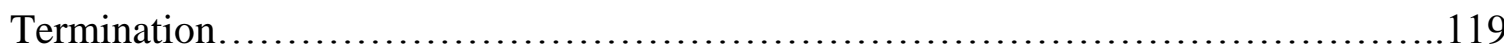

Table 14. Between-group Differences for CDI Mastery Status of Barriers to Treatment

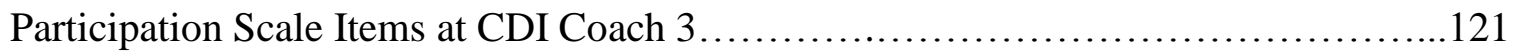

Table 15. Between-group Differences for Graduation Status of Barriers to Treatment Participation Scale Items at Midtreatment....................................... 122

Table 16. Ethnicity Differences on CDI Mastery Attainment.............................123

Table 17. Ethnicity Differences on PDI/Graduation Attainment.......................... 124

Table 18a. Ethnicity Mean Comparisons of Child Behavior Problems from First to Last Attended Session - T-Scores.......................................... 125

Table 18b. Ethnicity Mean Comparisons of Child Behavior Problems from First to Last Attended Session - Raw Scores.......................................... 125

Table 19. Ethnicity Differences for Therapy Attitude Inventory and Barriers to Treatment

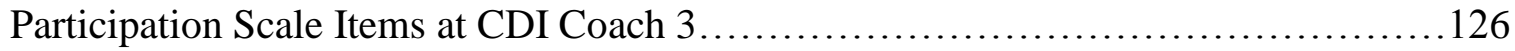




\section{List of Figures}

Figure 1. Eyberg Child Behavior Inventory - Intensity Scale T-Scores from

First to Last Session Attended for Incentive and Nonincentive Groups

Figure 2. Child Behavior Checklist - Externalizing Scale Scores Across

Treatment for Incentive and Nonincentive Groups............................. 128

Figure 3. Child Behavior Checklist - Internalizing Scale Scores Across

Treatment for Incentive and Nonincentive Groups....

Figure 4. Parenting Stress Index: Short Form - Total Stress Scale Scores Across

Treatment for Incentive and Nonincentive Groups............................ 130

Figure 5. Eyberg Child Behavior Inventory - Intensity Scale T-Scores from

First to Last Session Attended for Hispanic and Non-Hispanic Families.... 
List of Appendices

Appendix A. Family Demographic Form.......................................... 132

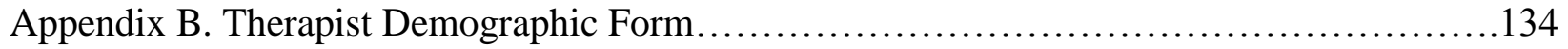

Appendix C. Session Record Form................................................... 136 
A Randomized Controlled Trial of Parent-Child Interaction Therapy with and without Incentives in a Community Mental Health Setting

Disruptive behavior problems (e.g., oppositional defiant disorder, conduct disorder) affect as many as 16\% of American children (American Psychiatric Association, 2013) and represent the most common reason for referral of youth to clinical settings (Loeber, Burke, Lahey, Winters, \& Zera, 2000; Sukhodolsky, Smith, McCauley, Ibrahim, \& Piasecka, 2016). The presence of such disorders in early childhood has a strong association with future delinquent behavior including substance use, vandalism, interpersonal and romantic partner violence, delinquency, and academic difficulty (Blair \& Diamond, 2008; Capaldi \& Clark, 1998; Gau et al., 2007; Loeber et al., 2000). Young children targeted for treatment can have long-lasting, positive outcomes which reduce the likelihood of later delinquent behavior compared to children with disruptive behavior problems who are not treated (Eyberg, Edwards, Boggs, \& Foote, 1998; Reid, Webster-Stratton, \& Hammond, 2003).

Evidence-based interventions provide effective forms of treatment for families of children with behavior disorders (Eyberg, Nelson, \& Boggs, 2008; Ward, Theule, \& Cheung, 2016). While traditional methods of therapy once involved working individually with children, they demonstrated little evidence of success (Kazdin, 1988). Newer therapies, however, have incorporated the child's parents into treatment and have shown efficacious outcomes (e.g., Parent-Child Interaction Therapy; Heinrichs, Kliem, \& Hahlweg, 2014; Schuhmann, Foote, Eyberg, Boggs, \& Algina, 1998). One of the most important factors for yielding family success in treatment is parental involvement (Kim, Munson, \& McKay, 2012). Specifically, outcomes in family therapy can vary significantly depending on the family's level of involvement, with greater levels of involvement yielding better outcomes (Chacko et al., 2009). A recent review of 
randomized controlled trials indicated that most studies measuring family involvement focused on family attendance engagement and homework adherence in treatment sessions (HaineSchlegel \& Walsh, 2015). Lower rates of homework completion have been highly correlated with a lower likelihood to graduate from treatment (Berkovits, O’Brien, Carter, \& Eyberg, 2010; Danko, Brown, Van Schoick, \& Budd, 2016; Lyon \& Budd, 2010) while lack of attendance has been highly correlated with a continuation of parent stress and child behavior problems (Barrett et al., 2008; Lyon \& Budd, 2010). Attendance and homework completion may serve as adequate measurements in identifying those at risk for terminating from treatment and not obtaining positive outcomes characteristic of evidence-based treatments.

The present study addressed the high rates of family failure to adhere to treatment protocol in an evidence-based treatment for severe child behavior problems in a community mental health agency. Two versions of the parent-training program Parent-Child Interaction Therapy (PCIT) were implemented to compare outcomes related to family adherence to treatment. Families were randomly assigned to either PCIT implemented as usual or PCIT in which key components of the treatment were incentivized for the families. Families in the incentive group had the opportunity to receive prizes for attendance at therapy sessions, completion of weekly skill-building homework assignments, and mastery of therapeutic skills. Groups were compared on rates of adherence to PCIT components and measurements of treatment success, and a cost analysis of the incentive model was conducted. Lastly, variables related to attrition for the sample were explored.

\section{Attrition}

Although successful evidence-based treatments are becoming increasingly available to families of children with disruptive behavior disorders, many clients never complete the therapy. 
Client attrition for psychological services has remained relatively high over time $(28-75 \%$; Barrett et al., 2008; de Haan, Boon, de Jong, Hoeve, \& Vermeiren, 2013; Rogers, 1951). Even the advancement of community-based mental health clinics has not altered the rates of attrition throughout the years (Barrett et al., 2008). Reported attrition rates in family therapy cover a large range, but the National Institute of Mental Health estimated approximately $60 \%$ of families who initiate treatment terminate in the first few sessions (NIMH, 2001). An additional $20-40 \%$ of families drop out of treatment in follow-up periods (Cohen, Mannarino, \& Knudsen, 2005; Flannery-Schroeder, Choudhury, \& Kendall, 2005). Since many families who fail to regularly attend therapy continue to struggle with the problem identified for treatment, these numbers remain concerning for a large portion of families who need mental health services (Dumas, Begle, French, \& Pearl, 2010; Lyon \& Budd, 2010).

\section{Homework}

Homework is defined as assignments given to clients where they are expected to practice skills learned in session for skill acquisition (Kazantzis, Deane, Ronan, \& L’Abate, 2005). Homework is a common component of therapy, and its use has been associated with beneficial treatment effects. Past research has shown that a therapist's use of homework and a client's adherence to homework in cognitive behavior therapy is associated with more positive treatment outcomes (Kazantzis, Whittington, \& Dattilio, 2010; Mausbach, Moore, Roesch, Cardenas, \& Patterson, 2010). Families in parent-training programs who completed more homework reported greater reductions in their child's problem behavior compared to less adherent parents (Kling, Forster, Sundell, \& Melin, 2010), had higher rates of satisfaction, and required fewer sessions to graduate from treatment (Danko et al., 2016). However, studies show that parents often complete only half of their homework assignments (Chacko et al., 2009). 


\section{Theory}

The high rates of therapy attrition and failure to complete homework assignments can be explained through the barriers to treatment theoretical model proposed by Kazdin and colleagues (Kazdin, Holland, Crowley, \& Breton, 1997). The theory claims that families experience a number of barriers that may play a role in their ability to be involved with therapy over time. These barriers include practical obstacles, perceptions of the treatment, and the relationship with the therapist (Kazdin, Holland, Crowley, \& Breton, 1997). The presence of barriers during treatment is believed to make a difference in family attendance above and beyond factors frequently measured in studies on attrition. Experiencing numerous stressors can interfere with a family's ability to remain engaged (e.g., complete homework, attend treatment; Baker-Ericzén, Hurlburt, Brookman-Frazee, Jenkins, \& Hough, 2010). Even when families are offered an evidence-based therapy that could reduce severe behavior problems in their children, more barriers have been associated with a family's inability to continue treatment over time (Lyon \& Budd, 2010).

\section{Parent-Child Interaction Therapy}

One of the leading evidence-based programs for young children (ages 2.5 - 7 years) with behavior problems is Parent-Child Interaction Therapy (PCIT). PCIT treats children's disruptive behavior by guiding parents and caregivers to utilize play therapy skills and discipline strategies with their children (McNeil \& Hembree-Kigin, 2010). Specifically, PCIT has two phases: ChildDirected Interaction (CDI) and Parent-Directed Interaction (PDI). In the first phase of therapy, CDI, the caregiver learns positive relationship-building skills incorporated into a daily 5-minute play situation. Once caregivers have mastered the CDI phase, PDI is implemented. In PDI, caregivers issue developmentally appropriate, direct commands followed by reinforcement for 
compliance (e.g., labeled praise, child-directed play) or issue a scripted time-out procedure for child non-compliance. Caregivers receive immediate feedback and coaching during the therapy to improve their skills. Feedback is given in real time to parents; clinicians sit behind a one way mirror to watch the parent-child dyad interact while providing feedback to the parent through a bug-in-ear system. In addition to practicing the skills in session, families are instructed to complete weekly homework sheets in which skills are practiced in the family's home for 5 minutes each day. Family practice is monitored through the completion of the homework sheets, and feedback is given based on issues that arise during in-home practice.

Coding for parent-child interactions is conducted almost every session to provide feedback to parents about their progress toward achieving mastery. During coding periods, families are monitored for 5 minutes without feedback from therapists. During CDI, therapists code for the presence of positive skills including labeled praise, reflections, behavior descriptions, imitation, and enjoyment while also tracking avoid skills including questions, commands, and negative statements. To hit CDI mastery, caregivers must have 10 labeled praises, 10 reflections, and 10 behavior descriptions as well as less than 3 total questions, commands, or negative statements within the 5-minute coding period. During PDI, therapists code a caregiver's ability to give effective commands with appropriate follow-through (e.g., waiting 5 seconds for compliance, giving labeled praises for compliance, giving warning statements, taking child to time-out). PDI mastery is obtained once a parent can give at least 4 commands within 5 minutes where $75 \%$ of those commands are effective and $75 \%$ of the commands have effective follow-through. Families will graduate from PCIT once the family has achieved both sets of mastery and learned how to roll out appropriate PDI skills at home and in public. The level of mastery parents obtain as they progress through treatment is a unique 
component of PCIT that distinguishes it from other parent training programs. As stated previously, families in PCIT cannot advance through treatment unless they have attained mastery (i.e., CDI, PDI). Families may learn the skills at different rates, but all families must reach mastery before they are allowed to graduate from treatment.

Longitudinal studies of evidence-based treatments for families of children with behavior disorders, such as PCIT, report impressive success rates of around 80\% (Eyberg et al. 2001; Gallagher, 2003). Research on PCIT has shown that the program increases positive parenting skills and child compliance rates, reduces levels of parenting stress, and significantly decreases child behavioral problems (Abrahamse et al., 2012; Schuhmann et al., 1998). The positive outcomes stemming from PCIT have also been shown to translate to the child's behavior in school settings (McNeil, Eyberg, Eisenstadt, Newcomb, \& Funderburk, 1991) and to siblings not receiving therapy (Brestan, Eyberg, Boggs, \& Algina, 1997). Treatment gains such as a reduction in child behavior problems and an increase in positive parenting skills have even been shown to maintain in PCIT for six years (Hood \& Eyberg, 2003). Unfortunately, the high success rates illustrated by PCIT are only demonstrated for those families able to complete the entire treatment protocol (Eyberg et al. 2001; Gallagher, 2003); families able to complete treatment have significant improvements in child behavior and life outcomes (e.g., success in school, reduced familial stress and disruptive behavior) compared to families who do not complete PCIT (Eyberg et al., 2008). Frequently, successful therapies such as PCIT have high termination rates (50\%, Werba, Eyberg, Boggs, \& Algina, 2006; 69\%, Lanier et al., 2011) and low rates of homework completion for those that remain in treatment (39.1\% - 77\%; Berkovits et al., 2010; Lyon \& Budd, 2010). 
Attrition in PCIT. Although PCIT yields powerful outcomes for families who graduate from treatment, implementation of PCIT in communities has indicated that many families discontinue treatment before meeting criteria for graduation (Pearl et al., 2012; Stokes, McNeil, \& Wallace, in press). Attrition in PCIT is defined as the discontinuation of treatment any time after attending the first therapy session and before completion criteria (stated previously) have been met (Fernandez \& Eyberg, 2009). A review of the literature indicates reported attrition rates for PCIT have greatly varied across outcome studies. Implementation studies for PCIT in different countries including Norway and China (Bjorseth, Wormdal, \& Chen, 2010; Leung, Tsang, Heung, \& Yiu, 2009) typically report low attrition rates which range from $15 \%$ to $23 \%$. U.S. samples report higher attrition rates in efficacy and effectiveness trials (McCabe \& Yeh, 2009; Pearl et al., 2012).

Efficacy trials, which are largely conducted in university-based settings under highly controlled conditions, generally report higher attrition rates than international PCIT studies, but still remain relatively low, ranging from $27.5 \%$ to $36 \%$ (Abrahamse et al., 2012; Fernandez \& Eyberg, 2009; Nixon, Sweeney, Erickson, \& Touyz, 2003; Werba et al., 2006). Even with the control of efficacy studies, some research has reported attrition rates as high as $57 \%$ (McCabe \& Yeh, 2009). Unfortunately, efficacy studies are limited in their capacity to determine a treatment's level of impact in community practice settings (Nash, McCrory, Nicholson, \& Andrasik, 2005). This can potentially overestimate client outcomes due to outside or external influences affecting treatment implementation (Nash et al., 2005).

In effectiveness trials (often more representative of typical clinical practice settings; Nash et al., 2005), where PCIT is implemented in less controlled community agencies using community therapists, PCIT outcomes have shown lower family attendance and homework 
completion than efficacy trials (Baker-Ericzén, Jenkins, \& Haine-Schlagel, 2013; Haine-Schlagel \& Walsh, 2015). Studies with U.S. samples have indicated attrition rates ranging between 60 to 70\% in PCIT families in these settings (Pearl et al., 2012; Stokes et al., in press). Frequently, these studies are tested in a mental health clinic, where dropout is high (Fowles et al., 2017). While researchers have hypothesized that home-based services lower attrition by reducing the number of barriers a family experiences to get to treatment, studies have indicated mixed results (Fowles et al., 2017; Lanier et al., 2011). Fowles and colleagues found that attrition rates were lower when PCIT was delivered within the home (Fowles et al., 2017), but another study comparing clinic and home-based PCIT services found no difference in attrition between groups (69\% attrition rate; Lanier et al., 2011).

High rates of attrition may be representative of barriers a family experiences in PCIT (Boggs et al., 2004). The barriers that may prevent regular attendance to a clinic for treatment include transportation (Boggs et al., 2004; Fernandez \& Eyberg, 2009), parent dissatisfaction waiting for services to begin (Boggs et al., 2004; Werba et al., 2006), and the slow pace of treatment progression (Boggs et al., 2004). Moreover, families report that busy schedules and additional stressors interfere with continual treatment attendance (Fernandez \& Eyberg, 2009). Irregularity of treatment attendance has also been predicted by parental factors of maternal distress (i.e., stress, depression; Werba et al., 2006), maternal stress (Capage, Bennett, \& McNeil, 2001), negative maternal verbal behavior toward her child (Fernandez \& Eyberg, 2009; Werba et al., 2006), low maternal intelligence (Fernandez \& Eyberg, 2009), paternal hostility (Stoolmiller, Duncan, Bank, \& Patterson, 1993), and low familial socioeconomic status (Fernandez \& Eyberg, 2009; Reyno \& McGrath, 2006; Werba et al., 2006). Some families also express unhappiness with the treatment approach which can reduce engagement and promote 
early termination (Boggs et al., 2004). Studies investigating patterns in parents who returned for follow-up assessments after treatment completion were found to be older and have higher scores in intelligence measures than their counterparts who failed to return for follow-up (Hood \& Eyberg, 2003). Yet, findings related to treatment completers may be unrepresentative of all families seeking treatment and generalization is cautioned. Predictors for family success in treatment include lower severity of child behavior problems and lower rates of maternal psychopathology (Reyno \& McGrath, 2006).

Inconsistent attendance and early withdrawal from mental health services do not allow for the successful outcomes otherwise viewed in treatment (Barrett et al., 2008; Lyon \& Budd, 2010). When families drop out of treatment, parenting stress levels and child behavior problems have been shown to remain (Boggs et al., 2004). When behavior problems remain or worsen over time due to early termination, families may view treatment unfavorably thus preventing them from seeking the services they need in the future. In turn, negative perceptions of therapy may limit future positive outcomes if families decide to pursue treatment again (Kazdin \& Whitley, 2006).

Homework in PCIT. Homework is used in PCIT as a reminder for parents to practice the skills they learn in session (McNeil \& Hembree-Kigin, 2010). Homework encourages caregivers to practice daily positive relationship building and maintenance (McNeil \& HembreeKigin, 2010) as well as consistency, predictability, and follow-through with their expectations. Moreover, homework aids with gradual generalization of skills outside of sessions and across settings (McNeil \& Hembree-Kigin, 2010). Even with the number of benefits gained from completing homework, families in PCIT may complete homework at highly variable and lower than recommended rates (Danko et al., 2016). 
While attrition has been largely studied in PCIT, studies on homework completion are less abundant. Families in PCIT have shown homework completion rates ranging from as low as $0 \%$ to as high as $86 \%$ indicating high variability in the dose of treatment each family receives when involved in therapy (Berkovits et al., 2010; Danko et al., 2016; Lyon \& Budd, 2010). The inconsistent levels of homework adherence could indicate significant variations in both quantity and quality of PCIT in each family. These differences can make it hard for researchers to understand why PCIT may not have worked for one family as lack of homework completion may confound other factors leading to family dropout if homework completion is not tracked. Additionally, families in PCIT may not regularly be completing homework over the course of treatment. Danko and colleagues (2016) found that all families in their study (both completers and dropouts) averaged $45 \%$ for homework completion.

Trends indicate that families who failed to complete PCIT were less likely to complete their homework than families who graduated from treatment (Berkovits et al., 2010; Danko et al., 2016; Lyon \& Budd, 2010). Families in PCIT who complete treatment have been shown to complete their homework a greater number of times during a week (5 - 6 times), while dropouts were more likely to complete their homework 0 to 1 time each week. Homework may serve as an indicator for lack of family involvement and satisfaction, as parents with higher satisfaction ratings (Therapy Attitude Inventory) completed more homework (Danko et al., 2016).

\section{Incentives}

Given the high rate of family barriers interfering with mental health service delivery in outpatient settings, innovative methods may be necessary to decrease attrition and homework noncompliance. While previous studies have focused on factors specific to families that are highly correlated with low attendance or homework completion, many of these factors cannot be 
changed during treatment (e.g., marital status, age, socioeconomic status) and therefore fail to produce an adequate solution to address the problem (Kazdin, Holland, Crowley, \& Breton, 1997). In line with the barrier-to-treatment theoretical model, researchers have instead urged for the research to shift focus on factors that can be addressed in treatment (Armbruster \& Kazdin, 1994; Kazdin, Holland, Crowley, \& Breton, 1997). This shift places a greater responsibility on therapists and agencies to target concerns rather than placing emphasis on family characteristics or short-comings. One such method that has yielded success for individuals at high risk for nonadherence to treatment protocol (i.e., substance abusers) is the use of incentives.

Incentives (e.g., contingency management) have been used in a number of ways to increase involvement in activities that may be difficult for individuals to initially start (e.g., mental health services; Ryan \& Deci, 2000). Based on the principles of behavior modification, incentives are provided to clients when the target behavior is achieved, but incentives are withheld all other times (Bride \& Humble, 2008). Incentives were used in this study as rewards contingent upon attaining a desired behavior in treatment (i.e., timely attendance, homework completion, attaining mastery criteria). Incentives have been used similarly and with successful outcomes in substance abuse research to increase behaviors in a population faced with a number of barriers.

Incentives with substance abuse. A rich body of research on incentives in treatment has been conducted in the area of substance abuse. Incentives are given to increase behaviors such as attendance in therapy sessions or decrease problematic behaviors such as drug use. For example, Iguchi, Belding, Morrel, and Lamb (1997) incentivized opiate-dependent individuals with vouchers redeemable for retail goods. The researchers found that the drug users were more likely to complete individualized treatment components and have drug-free urine samples when given 
vouchers (Iguchi et al., 1997). Other patient behaviors such as abstinence from substance use and modified verbalizations (e.g., identifying negative outcomes from drug use) were shaped with the implementation of incentivized treatment (Petry, 2000; Petry et al., 1998).

In other studies done by Petry and colleagues, the researchers incentivized clean urine samples and attaining treatment goals targeted at long-term success (e.g., enrollment and attendance in Alcoholics Anonymous, improvement in relationships through scheduling positive activities) with opportunities to win "draws" from a prize bowl (Petry et al., 2004). Two hundred fifty slips of paper were drawn from a prize bowl with half the slips having no prize reward, and the other half having a prize reward ranging significantly in monetary value. Petry found the mystery prize bowl method to yield positive results (Petry et al., 2004).

In another method, Pollastri and colleagues found that low cost incentives (i.e., calling cards or gift certificates) reduced unanticipated no-shows for appointments by approximately $50 \%$ for 415 adults with a dependence on cocaine or opioids (Pollastri, Pokrywa, Walsh, Kranzler, \& Gelernter, 2005). Incentives used with drug-abusers such as \$3 gift certificates, vouchers exchangeable for goods and services, and take-home methadone doses were found to increase attendance for intake sessions, therapy appointments, and skill-based training programs (Carey \& Carey, 1990; Chutuape, Katz, \& Stitzer, 2001; Kidorf, Stitzer, Brooner, \& Goldberg, 1994; Silverman, Chutuape, Bigelow, \& Stitzer, 1996). Giving incentives to clients exhibiting high-risk behavior can increase the likelihood of attending treatment and engaging in therapeutic activities.

Incentives with family programs. Although incentivized treatment has been wellstudied with patients suffering from drug dependencies, a dearth of research exists on utilizing treatment incentives in family programs. Some limited research has shown the potential for 
success utilizing this method (Hayes, Efron, Richman, Harrison, \& Aguilera, 2000). Clients in a Hayes and colleagues' study signed a contract stating their agreement to attend sessions and that if they attended four consecutive appointments, they would receive a $\$ 30$ coupon (Hayes et al., 2000). Although only three families were included in the study, all families significantly increased appointment attendance after the incentive was introduced after baseline. Expansion of this pilot study is necessary to determine if incentives work at a larger level for families with children who have behavior disorders.

In a large study, researchers from Harvard University implemented incentive programs designed to bolster student achievement as measured by test scores, grades, literacy rates, and classroom behavior (Frye, 2011). Random assignment to reward programs was conducted in 43 Dallas, 143 New York City, 17 Washington DC, and 70 Chicago schools. Reward programs varied from receiving $\$ 2$ per book read to $\$ 25-\$ 50$ per test completed, with prizes up to $\$ 100$ every two weeks, and up to $\$ 250$ per report card (dependent on grades obtained). Working with families and children, Frye did not find powerful outcomes for academic achievement, however cash incentives used with families for children's behavior and attendance did produce positive effects (e.g., Washington DC where students could earn up to $\$ 100$ every two weeks). Frye concluded that behavior change can occur when desired behaviors are incentivized (rather than end products) as the specific actions are more likely to occur due to the immediate outcome and the action's tangible nature (Frye, 2011). Positive outcomes found in family incentive research may yield the same results when expanded to evidence-based family-focused treatments.

One older study also found significant positive effects for incorporating reinforcement into their treatment protocol such that targeted treatment goals were obtained (Eyberg \& Johnson, 1974). The study evaluated the effects of a contingency management program on 
parental cooperation (i.e., attendance, completion of assigned data, therapist ratings of cooperation) and treatment outcome (i.e., parental observation data, parental report measures, home and lab observational data) in family therapy for severe child behavior problems (Eyberg \& Johnson, 1974). Manipulated contingency conditions included client fee, therapy time, and telephone time for four groups. Treatment was targeted for child behavior problems specific for each family. Although unique to each client, core components of treatment included principles based on behavior modification and social learning theory, defining the child problem behaviorally to the family, gaining baseline data, administering consequences to a child exhibiting the problem behavior, reinforcing the child when multiple days without problem behavior were present, and giving parents more control of designing, modifying, and tampering the treatment over time. All clients paid for 12 therapy sessions before initiating therapy, but the contingency group paid an additional 12 session fees which they were able to gain back (per session) through attendance and completing over half of the assignments given after each session. Moreover, these families were only allowed to attend therapy and receive telephone consultation if they completed assignments. Calls (three per week) and sessions (once weekly) were canceled with the failure to complete assignments. The researchers found that families in the contingency group completed more assignments, had higher cooperation rating by therapists, had more child problem behaviors treated than families assigned to the noncontingency group (Eyberg \& Johnson, 1974).

One limitation to the study by Eyberg and Johnson (1974) was the decision to exclude families who terminated early from treatment from the measurement of outcomes. While it is important to determine if contingencies increased treatment adherence, eliminating a large portion of participating families due to a failure to complete treatment potentially skewed the 
outcomes and over-represented treatment effectiveness. Specifically, this study did not have a representative sample of participating families in outpatient community mental health agencies because all dropouts were eliminated from study participation. Remaining families in both groups represented a selection bias as they attended over $90 \%$ of scheduled therapy sessions. It is important to explore the most representative sample of families in outpatient care to better understand the issues that arise and determine effective evidence-based methods to increase treatment adherence and the chance each family will be successful.

Concerns about incentives. Some concerns remain related to attrition and treatment incentives. Specifically, one concern focuses on if incentives drop too low in monetary value. A few studies have shown that if an incentive is too low, the effectiveness of the incentive is lost (Silverman, Chutuape, Bigelow, \& Stitzer, 1999). Sacks and colleagues found that low-level incentives $(\$ 10)$ for mental health visits did not increase low-income, pregnant women's likelihood of attending treatment for depression (Sacks, Green, Burke, \& Owen, 2015). Quality of mental-health services was not detailed in the study leading to the possibility that evidencebased, short-term treatments were not used. Therefore, the lack of improvement may have led to dropout. In turn, many of these mothers already had children and therefore going to treatment without childcare may have been a major barrier. In another study, Silverman and colleagues (1999) exposed methadone patients to three voucher-based programs where they could earn $\$ 0$, $\$ 382$, or $\$ 3,480$ for producing cocaine-free urine samples during mandatory testing. Significant effects were found in which increased voucher magnitude was associated with increased length of time abstinent and number of drug-free urine samples produced (Silverman et al., 1999). In this sample, individuals had already undergone a voucher-based treatment in which they had failed to produce drug-free urine samples (vouchers totaling \$1,155). These individuals in 
smaller voucher-based groups may not have been motivated by lower costs due to previous exposure to incentivized treatment. However, these results for low-level incentives are inconclusive as small incentives have been shown in more recent studies to make clinically significant change with as little as $\$ 15$ in vouchers per week for clean drug tests (Iguchi et al., 1997; Petry et al., 2004). Results remain mixed for low-rate incentives for drug-users, and this pattern remains for research outside of abstinence-based programs.

A second major concern has been raised for the effectiveness of incentives in non-drugbased programs. For example, Dumas and colleagues examined parental involvement in an eightweek parent-training program intended to increase positive parent-child interactions (Dumas et al., 2010). This eight session prevention program was incentivized with $\$ 3$ rewards given for the first two sessions, while future sessions steadily increased in monetary value with the last two sessions reaching \$15. Dumas and colleagues (2010) found that incentivizing attendance encouraged a number of parents to sign up compared to non-incentivized families, but it did not have a significant effect on attendance in the sessions between groups. To understand the lack of findings in this study, it is important to note that research on universal preventative care has shown historically low rates of attendance and enrollment (Baker, Arnold, \& Meagher, 2011). Families not in crisis may lack motivation to seek or remain with services. Furthermore, since starting incentives were low, incentives may not have provided enough motivation for families to remain throughout the program. Findings from prevention programs may not apply to treatments for children with behavior disorders as monetary incentives may be the only motivator for prevention attendance. Comparatively, families with children experiencing severe behavior problems may be motivated by the significant stress to attend the first few sessions of treatment before requiring an additional incentive to remain in therapy. 
A third concern in the literature is that the inclusion of incentives removes a client's intrinsic motivation to engage in therapy and that this may have a negative impact for the development of therapeutic skills (Kohn, 1993). While this argument has been raised, recent research has not found this outcome to be true (Heinrichs \& Jensen-Doss, 2010). In looking at outcomes in a preventive parenting program, Heinrichs and Jensen-Doss (2010) found mixed results for parenting skills for both parents paid and unpaid for attending therapy at 1 and 2 years following the original assessment. The researchers argued that incentives had no impact on client outcomes and instead, incentives attracted families who may not have otherwise engaged in services therefore providing services to a greater range of families (Heinrichs \& Jensen-Doss, 2010).

A fourth concern is the use of a practices that were once acceptable in older research, but may now present as ethically problematic and would not likely be replicable in current clinical and research practices. Specifically, in Eyberg and Johnson's work (1974), researchers refused to speak to or provide treatment to a client due to a failure to complete homework assignments. This action may now been seen as a significant concern as it may place a family's health at risk. Creating a more ethically sound solution such as incentivizing desired behaviors (e.g., homework completion, attendance) is needed for the ultimate goal of increasing family success in treatment.

Cost of incentives versus cost of attrition. One additional concern for agencies when implementing incentives may be the direct cost of the rewards. Large cuts in the federal budget to mental health care services in recent years have had severe consequences on the utilization of community mental health agencies and their revenue (Honberg, Diehl, Kimball, Gruttadaro, \& Fitzpatrick, 2011). For example, the cuts from the government reduced services Medicaid-funded individuals could utilize; these cuts led to a greater number of mental health care agencies no 
longer being able to take Medicaid-funded clients (Honberg et al., 2011). Reasonable concerns may arise related to incentives as further costs to the agency could have significant detrimental effects on staffing, building maintenance, or therapy materials. In order to justify dedicating expenditures on incentives, research demonstrating the positive outcomes is necessary.

Due to the negative consequences that can occur from children who remain untreated such as academic problems, substance dependence, or reliance on mental health services as adults (see de Haan et al., 2013, for a review), it was believed that the reduction of early termination from treatment such as PCIT would result in more cost effective care. Not only do many families who drop out of therapy experience direct negative outcomes, but the failure of the families to complete or attend therapy can have detrimental outcomes to the community mental health centers as well (Barrett et al., 2008). Failure of a client to attend therapy prevents staff from being able to charge for that session as no-shows and last minute cancellations prevent staff from filling the session time. Since attrition rates are high, this continuous occurrence can place strain on many community clinicians. Missed appointments can limit billable hours, reduce staff salaries, prevent other families in need from attaining treatment, increase waitlist times, and with continuous attrition, can dampen staff morale and increase turnover (Joshi, Maisami, \& Coyle, 1986; Klein, Stone, Hicks, \& Pritchard, 2003). Over time, families may develop a negative perception of both the agency and therapy in general if their children's behavior problems are not remedied. Increasing client engagement and success is imperative to a productive and effective community mental health system.

\section{Purpose of the Study}

Only 20 percent of families in need of mental health services are targeted and receive treatment every year (U.S. Public Health Service, 2001). Of those who seek services, the 
majority drop out of treatment in the initial sessions (NIMH, 2001), with a significant portion terminating treatment early over the course of an intervention (Cohen et al., 2005; FlannerySchroeder et al., 2005). Effectiveness studies evaluating PCIT implementation in community settings demonstrate concerning attrition rates as high as 70\% (Lanier et al., 2011; Pearl et al., 2012; Stokes et al., in press). There is a need for evidence-based retention strategies throughout the course of PCIT (Fernandez \& Eyberg, 2009). Failure to address this continuing problem will result in unsuccessful treatment of families, poor outcomes for children with behavior problems, further costs associated with the poor life trajectories of these children (e.g., incarceration, no long term employment), and lost income for mental health facilities (Capaldi \& Clark, 1998; Garfield, 1994). The goal of this research study was to determine if treatment incentives would increase family adherence to treatment (e.g., timely session attendance, homework completion) and success of families (e.g., CDI and PDI mastery, lower child behavior problems, lower parental stress) in PCIT, as well as increase the cost effectiveness of this intervention within a community-based mental health system (Abrahamse et al., 2012; Schuhmann et al., 1998).

The present research evaluated differences between PCIT implemented as usual compared to PCIT with incentives in a community mental health agency. Families in this study were representative of a lower socioeconomic status in a community sample. Families in the incentivized group received rewards (i.e., mystery prize bags) for PCIT components that addressed both involvement and success, such as treatment attendance, homework completion, and milestone attainment. These variables were measured throughout the course of treatment to determine if differences arose between groups. No researchers have previously attempted to incentivize components of PCIT (e.g., homework, attendance) in a restricted randomized 
controlled trial with a large number of clientele. The present study could have important clinical implications for decreasing attrition in PCIT and other evidence-based interventions.

\section{Aims}

\section{Aim 1}

Evaluate whether families referred to PCIT who were provided incentives for therapy had greater adherence to treatment components than families receiving PCIT as usual.

Previous research has shown that large numbers of families enrolled in therapy drop out over the course of treatment due to a number of perceived barriers (Owens et al., 2002). PCIT, in particular, has demonstrated high rates of dropout in research and in clinical settings (Lanier et al., 2011; Werba et al., 2006). Despite a wide array of barriers, incentivizing components of treatment has been shown to be effective in increasing a number of healthy behaviors in high risk families (see Klein, 2014, for a review). Specifically, large gains have been made in drug dependency research where attendance and positive strength-based behaviors have increased from the implementation of incentivized treatment (Iguchi et al., 1997; Petry et al., 2004). Research by Hayes and colleagues (2000) used incentives to encourage low-income families to attend four therapy sessions in a row. Families increased their consistency and attendance of appointments during the study (Hayes et al., 2000). A number of similar studies have shown increased attendance as well as completion of assigned work when incentives were provided (Carey \& Carey, 1990; Petry et al., 2004). Given these findings, families in the present study received incentives for behaviors that supported the attainment of treatment goals. Incentives were specifically expected to increase family involvement. Hypotheses related to involvement were measured in the following ways: 
A. Families in the PCIT plus incentives group were expected to require fewer sessions to obtain CDI mastery than families receiving PCIT as usual.

B. Families in the PCIT plus incentives group were expected to need fewer weeks to obtain CDI mastery.

C. Families in the PCIT plus incentives group were expected to drop out of treatment before CDI mastery less often than families receiving PCIT as usual.

D. Families in the PCIT plus incentives group were expected to have a smaller percentage of no-shows to scheduled therapy sessions than families receiving PCIT as usual.

E. Families in the PCIT plus incentives group were expected to have a smaller percentage of cancellations for scheduled therapy sessions than families receiving PCIT as usual.

F. Families in the PCIT plus incentives group were expected to have a smaller percentage of sessions in which they arrived late (10 minutes or more) than families receiving PCIT as usual.

G. Families in the PCIT plus incentives group were expected to complete more homework assignments (both paper and audio recordings) per week than families receiving PCIT as usual.

\begin{abstract}
$\operatorname{Aim} 2$
Evaluate whether families referred to PCIT who were provided incentives for therapy had greater success in treatment than families receiving PCIT as usual.

In a number of research studies, PCIT has shown significant effects on child behavior problems for internalizing and externalizing behaviors (Chase \& Eyberg, 2008) and parental stress levels (Matos, Bauermeister, \& Bernal, 2009). One factor necessary for graduation from PCIT is the reduction in child behavior problems (as measured by the Eyberg Child Behavior
\end{abstract}


Inventory; ECBI) to below clinically significant levels (McNeil \& Hembree-Kigin, 2010).

Changes in ECBI scores across PCIT outcome studies indicated drops in a child's intensity of problem behavior by $29 \%$ and in problem behaviors by $61 \%$ (Goldfine, Wagner, Branstetter, \& McNeil, 2008). The ECBI is an appropriate measure for indicating treatment effects due to its sensitivity to change in behavior problems over time (Eyberg \& Robinson, 1983). However, families progress through PCIT and master therapeutic skills at different time points due to a number of factors. Many times, PCIT can be another burden in the lives of these highly stressed families (Timmer, Urquiza, Zebell, \& McGrath, 2005). Inconsistencies in attendance and dropout have been shown to reduce the likelihood for positive outcomes to occur from treatment (Barrett et al., 2008; Lyon \& Budd, 2010). It was hypothesized that incentives used in treatment would keep families motivated to attend sessions and therefore reach greater gains with respect to targeted problem behaviors (Eyberg \& Johnson, 1974).

Hypotheses related to success were measured in the following ways:

A. Families in the PCIT plus incentives group were expected to attain CDI mastery at a higher rate than families receiving PCIT as usual.

B. Families in the PCIT plus incentives group were expected to attain PDI mastery at a higher rate than families receiving PCIT as usual.

C. Families in the PCIT plus incentives group were expected to graduate from PCIT at a higher rate than families receiving PCIT as usual.

D. Families in the PCIT plus incentives group were expected to have a greater decrease in child behavior problems (Eyberg Child Behavior Inventory; Eyberg \& Pincus, 1999) than families receiving PCIT as usual. 
E. Families in the PCIT plus incentives group were expected to have a greater decrease in externalizing and internalizing behavior problems (Child Behavior Checklist; Achenbach $\&$ Rescorla, 2001) than families receiving PCIT as usual.

F. Families in the PCIT plus incentives group were expected to have a greater decrease in parenting stress levels (Parenting Stress Index: Short Form; Abidin, 1990) than families receiving PCIT as usual.

\section{$\operatorname{Aim} 3$}

A cost analysis was conducted to determine if there was any financial difference between the PCIT plus incentives group and the PCIT as usual group in the community mental health agency.

Budget cuts to public health funding occur regularly and pose a significant challenge to community mental health agencies (Honberg et al., 2011; Lippman, 2012). Community agencies have to closely monitor their spending or risk being shut down. Unfortunately, these cuts are particularly hard for low-income families who have no private options for finding mental health services (Lippman, 2012). The success of an agency depends on having full caseloads of clients who attend sessions consistently, as no-shows and cancellations greatly reduce billable hours (Joshi et al., 1986; Klein et al., 2003). It is expected that incentives will increase client attendance and therefore increase revenue for the community mental health agency. In turn, the increased revenue will offset the costs of providing incentives for PCIT clients making incentivized treatment a more sound use of resources for the agency. The analysis will be based on Drummond and colleagues' calculations for economic evaluations of health care programs (Drummond, O’Brien, Sculpher, Stoddart, \& Torrance, 2005).

\section{Exploratory Question}


Which variables were most highly associated with attrition from PCIT?

Previous PCIT literature has indicated trends in early termination with disproportionately more families with lower socioeconomic statuses dropping out early (Harwood \& Eyberg, 2004). This finding has been found repeatedly in other studies of family therapy, along with additional associations for low treatment adherence including minority status, maternal age, single-parent status, parental depression, child age and sex, and greater child conduct problems (Bean \& Talaga, 1992; Hoffman, Crnic, \& Baker, 2006; Kazdin, Holland, \& Crowley, 1997; Kazdin \& Mazurick, 1994; Singh, Janes, \& Schechtman, 1982; Werba et al., 2006). In addition, findings have shown barriers to treatment participation and parental satisfaction may also contribute to a family's likelihood of early termination from PCIT (Lyon \& Budd, 2010). The current study assessed the role of incentives and other variables commonly associated with early termination (Capage et al., 2001; Fernandez \& Eyberg, 2009; Reyno \& McGrath, 2006; Stoolmiller et al., 1993; Werba et al., 2006).

\section{Method}

\section{Participants}

Families. Families were recruited to participate in the present study prior to enrollment. Upon consenting to the project, families were then randomly assigned to one of two groups: PCIT delivered as usual or PCIT with the addition of incentives for completing specified treatment components. Random assignment occurred through a computer-generated assignment program. When each family contacted the community mental health agency, they were assigned a computer-generated identification number. Odd numbers from the computer were assigned to the PCIT plus incentives group, while even numbers were indicative of the PCIT as usual group. A supervisor double checked the group each family was assigned based on the community 
identification number before treatment started (i.e., during the first assessment). A new

participant number was then assigned to the family for the purposes of the study. The community identification number was not included in data collection. A total of 84 parent-child dyads were enrolled in the study. Participants were recruited from Prevention Early Intervention, Riverside University Health System - Behavioral Health, Preschool 0 - 5 Programs, including SET-4School and Mobile Prevention and Early Intervention in Riverside, California.

All data were collected at the Riverside facilities. De-identified data were shipped to West Virginia University through the Parent-Child Interaction Therapy lab run by Dr. Cheryl B. McNeil for analysis. Families interested in receiving family therapy were recruited to participate in the research study during their initial contact with the Riverside facilities. Families were randomly assigned and informed of their receipt of incentives after signing the informed consent. Inclusion criteria for this study included age ranges for children from 2 to 7 years and caregiver's legal custody of the child. The adult was required to be a primary caregiver of the child and be 18 years of age or older. All families adhering to these standards were eligible to participate. Only families consenting to the study's procedures were enrolled in the study. Families denying participation $(n=4)$ in the study were not denied services. Families who were receiving treatment and decided to no longer participate in the study were allowed to continue with services with no penalty to them.

Clinicians. Twenty five PCIT-trained therapists worked for Riverside University Health System - Behavioral Health, Preschool 0 - 5 Programs, including SET-4-School and Mobile Prevention and Ealy Intervention at the initiation of the study. Of those, 11 PCIT-trained clinicians agreed to participate in the current study and undergo CITI training to meet IRB protocol. However, two clinicians left the agency prior to starting family enrollment. Therefore, 
only nine clinicians participated in the study. Eight of the nine clinicians delivered PCIT to both treatment groups, while the last clinician provided supervision of cases and monitored study protocol. At the start of data collection in April, 2016, seven of the nine therapists were licensed clinicians. Eight of the clinicians were full-time with approximately 15 cases per week totaling 120 PCIT families seen in one week.

Clinicians of the community mental health agency provided services in mobile clinics $(n$ $=25)$ and an outpatient clinic $(n=59)$. No home-based services were provided. Clinicians were trained in the present study protocol to collect data and prompt incentive reminders through a designated script. The supervising clinician was informed and trained in monitoring responsibilities. Clinicians participated in a weekly supervision meeting to discuss study questions and monitor protocol fidelity. The primary investigator participated in the supervision through a call during this time. The primary investigator and supervisor in the study underwent bi-weekly meetings to discuss, problem solve, and consult on study rollout. These meetings transitioned to monthly appointments over the course of the study, although additional appointments were made, as necessary.

\section{Measures}

Measures were selected based on the research questions posed and from feasibility of the community mental health agency. Measures on child behavior problems and parenting stress were already utilized by the agency. Measures were additionally selected for their relevance to PCIT literature, their evidence-base, and the low cost to the community agency (e.g., Barriers to Treatment Participation Scale, Therapy Attitude Inventory).

Demographic Form. The Demographic Form was completed by caregivers to cover demographic information regarding the caregiver and the child. The caregiver answered details 
about the caregiver's sex, age, ethnicity, and yearly income. The questionnaire also covered information about the child including sex, age, and the relationship of the caregiver to the child. Another Demographic Form was also completed by therapists to measure sex, ethnicity, years as a clinician, and years delivering PCIT.

Barriers to Treatment Participation Scale. The Barriers to Treatment Participation Scale (BTPS; Kazdin, Holland, Crowley, \& Breton, 1997) is a 58-item measure assessing psychological and practical barriers to client engagement which was completed by caregivers after the third CDI Coach session, at midtreatment (after CDI mastery), and posttreatment (graduation). Originating from the barriers-to-treatment theoretical model, this measure recognizes families experience a number of barriers that may prevent them from attending treatment and aims to determine the most influential factors preventing treatment attendance for each family. Items 1 through 44 assess caregivers' perceived barriers to treatment (e.g., "I am too tired after work to go to sessions") using a 5-point Likert scale ( $1=$ Never a Problem; 5 = Very Often a Problem). Items 45 through 58 assess barriers on a binary (yes/no) scale. For the purposes of this study, these last items were not examined. The BTPS yields four subscale scores calculated from summing measure items: Competing Activities/Life Stressors (20 items), Relevance of Treatment (8 items), Relationship with Therapist (6 items), and Treatment Issues (10 items). In the present study, each individual item was examined independently, each subscale score was calculated (Competing Activities/Life Stressors, $\alpha=.78$; Relevance of Treatment, $\alpha=$ .37 ; Relationship with Therapist, $\alpha=.06$; Treatment Issues, $\alpha=.54)$, and a total score was generated $(\alpha=.78)$. BTPS has shown acceptable inter-item consistency (Kazdin, Holland, Crowley, \& Breton, 1997) and other versions of the BTPS have been shown to have good levels of internal consistency (Nanninga, Jansen, Kazdin, Knorth, \& Reijneveld, 2016). Although the 
measure has not been standardized with Hispanic populations, the following alphas were generated in the present study: Competing Activities/Life Stressors (Hispanic: $\alpha=75$.; NonHispanic: $\alpha=.79$ ), Relevance of Treatment (Hispanic: $\alpha=.47$; Non-Hispanic: $\alpha=.19$ ), Relationship with Therapist (Hispanic: $\alpha=.05$; Non-Hispanic: $\alpha=.05$ ), and Treatment Issues (Hispanic: $\alpha=.53$; Non-Hispanic: $\alpha=.46$ ), Total score (Hispanic: $\alpha=.76$; Non-Hispanic: $\alpha=$ $.80)$.

Therapy Attitude Inventory. The Therapy Attitude Inventory (TAI; Eyberg, 1993) is a 15-item measure completed by caregivers during the third CDI Coach session. The TAI measures family perceptions of progress in treatment, relationship with the therapist, and satisfaction with treatment. Each item is rated on a 5-point Likert scale with 1 indicating dissatisfaction or worsening of indicated problems and 5 indicating a high satisfaction or improvement in indicated problems. A total score can be calculated from the summation of all the items. For the present study, each individual response was independently studied in addition to total scores $(\alpha=.78)$. Previous research has stated this measure is not prone to ceiling effects that otherwise frequent satisfaction measures (Brestan, Jacobs, Rayfield, \& Eyberg, 1999). The TAI has shown adequate internal consistency, test-retest reliability, and discriminative validity (Brestan et al., 1999; Eisenstadt, Eyberg, McNeil, Newcomb, \& Funderburk, 1993). Although the measure has not been standardized with Hispanic populations, the following alpha was generated: Total score (Hispanic: $\alpha=.78$; Non-Hispanic: $\alpha=.77$ ).

Eyberg Child Behavior Inventory. The Eyberg Child Behavior Inventory (ECBI; Eyberg \& Pincus, 1999) is a 36-item measure completed by the caregiver of a child aged 2 through 16. Caregivers rated child behaviors on two scales for an Intensity and Problem score. For the Intensity rating, caregivers rated a range of child behaviors on a seven-point Likert scale 
indicating how frequently the behavior occurred ( 1 = Never, 7 = Always $)$. The same behaviors were also rated by caregivers as problematic (Problem scale) by marking "yes" or "no." Caregivers completed the measure during each attended session.

Previous studies on the ECBI have shown strong psychometric properties. The ECBI was standardized based on the U.S. population Census data in 1999 (Colvin, Eyberg, \& Adams, 1999). The ECBI had been shown to have strong test-retest reliability over 10 months for both Intensity $(r=.75, p<.01)$ and Problem scores $(r=.75, p<.01$; Funderburk, Eyberg, Rich, \& Behar, 2003). Internal consistency is high for both Intensity $(\alpha=.94)$ and Problem scales $(\alpha=$ .93; Colvin et al., 1999). Interrater reliability between maternal and paternal Intensity ratings and Problem ratings were both acceptable $r=.69$ and .61 , respectively (Eisenstadt, McElreath, Eyberg, \& McNeil, 1994). Construct validity on the ECBI has been found to be strong with correlations present between the ECBI and the Child Behavior Checklist's Externalizing scales (Boggs, Eyberg, \& Reynolds, 1990). The ECBI is sensitive to treatment effects and is regularly used for outcome measurement in PCIT. In addition, the ECBI has been used in a number of child populations with developmental disabilities (Sofronoff, Leslie, \& Brown, 2004). Only the Intensity scale was utilized in this study.

Child Behavior Checklist. The Child Behavior Checklist (CBCL; Achenbach \& Rescorla, 2001) is a Likert-scale questionnaire completed by caregivers to assess child behaviors and emotions. Responses for each item range from 0 (Not Present) to 2 (Often True). The three main scales utilized in the CBCL include the assessment of internalizing problems (Internalizing), externalizing behaviors (Externalizing), and overall problem behaviors (Total). The Internalizing subscale measures areas such as emotions, anxiety, depression, and physical pain. The Externalizing subscale measures areas such as aggression, noncompliance, and 
attention difficulties. The CBCL has two forms for children ages $1 \frac{1 / 2}{2}-5$ (99 items) and $6-18$ (112 items). Caregivers completed this measure at pretreatment (intake session), at midtreatment (i.e., CDI mastery), and at treatment graduation.

The CBCL has shown strong utility for distinguishing clinical and nonclinical populations (Seligman, Ollendick, Langley, \& Baldacci, 2004). Strong test-retest reliability ratings ranging from .95 to 1.0 have also been reported in past research as well as strong measures of internal consistency for both the Internalizing and Externalizing scales with scores of .90 and .94, respectively (Achenbach \& Rescorla, 2001). The CBCL's DSM-Oriented scales have good test-retest reliabilities with Cronbach alphas from .75 to .84 (Achenbach, Dumenci, \& Rescorla, 2003). In turn, these scales have shown strong reliability coefficients ranging from .71 to .89 (Nakamura, Ebesutani, Berstein, \& Chorpita, 2009). The Internalizing and Externalizing scales were analyzed in the current study.

Parenting Stress Index: Short Form. The Parenting Stress Index: Short Form (PSI:SF; Abidin, 1990) is 36-item parent self-report measure derived from the Parenting Stress Index. The PSI:SF was shown to be highly correlated with the longer PSI version (120 items), $r=.94$ (Abidin, 1990). Caregivers reported on stressors related to aspects of raising their child and responsibilities from parenting. The PSI:SF can be used for children ranging in age from 3 months to 12 years. Four subscales are used in the PSI:SF including ratings of Parental Distress, amount of Parent-Child Dysfunction, perceptions and reported levels of Difficult Child, as well as Total Stress levels. Caregivers completed this measure at pretreatment (intake session), at midtreatment (i.e., CDI mastery), and at treatment graduation.

The PSI:SF has shown strong test-retest reliability for the Total Stress score with a coefficient of .86 (Abidin, 1995). Other studies have shown the strength of the PSI through 
reports of reliability for all scales $\alpha>.60$ (Whiteside-Mansell et al., 2007) as well as for the parent category $(.55-.80)$ and the child category $(.62-.70$; Loyd \& Abidin, 1985). Moreover, the PSI scales have shown strong reliability coefficients for family and child characteristics as well as distress (Whiteside-Mansell et al., 2007). Only the Total Stress scale was used in the present study.

\section{Dyadic Parent-Child Interaction Coding System-IV. The Dyadic Parent-Child} Interaction Coding System, fourth edition (DPICS; Eyberg, Nelson, Ginn, Bhuiyan, \& Boggs, 2013) is an observational measurement method designed to assess parent and child interactions. Parent-child dyads are coded during play situations for three separate 5-minute interactions. These interactions consist of scenarios in which the child leads the play (Child-Led Play), the parent leads the play (Parent-Led Play), and then the parent instructs the child to clean up the toys by himself or herself (Clean Up). Parent verbalizations (e.g., labeled praise, behavior description, reflection, command) and behaviors (e.g., imitation, enjoyment) are coded throughout these interactions. Child compliance to commands is also recorded.

The DPICS has strong reliability and validity for sessions coded live or coded later using video recordings (Eyberg et al., 2005). Measurements of inter-rater reliability have demonstrated adequacy (Robinson \& Eyberg, 1981). DPICS has been used to discriminate clinically referred families and children from normal comparisons (Eyberg, Nelson, Duke, \& Boggs, 2005; Robinson \& Eyberg, 1981). The present study utilized a measurement of parental negative verbalizations (i.e., negative talk, commands, questions) and positive verbalizations (i.e., labeled praises, reflections, behavioral descriptions), and were recorded at all attended PCIT coaching sessions. 
Homework. The participants were expected to complete homework sheets standard to PCIT protocol. These sheets required parents to fill out their participation in play and discipline procedures for their child. Families were expected to record whether they participated in the play each day, which toys were utilized for play, and the number of time-out episodes each day. Families were also asked to audiotape these play sessions using their mobile devices. If families did not have mobile devices, they were provided with audio recorders that they needed to bring to each session for the therapist to review. The supervising clinician tracked and monitored the distribution and return of audio recorders for each family. The frequency of days that families completed their homework sheet and audio recordings were analyzed in the current study.

Session Record Form. Therapists in this study recorded information for each participating family every session including the family's study ID number, the session number, if the participant attended (on time, was late, canceled, or no-showed), the last time since the family attended treatment, if homework was completed, and how many incentives (i.e., mystery prize bags) the family received for the session. This data collection was used to track family fidelity throughout the course of therapy. Dependent variables for this measure included attendance status, frequency of homework completion (paper form, audio recordings), and attainment of mastery or graduation.

\section{Procedure}

Regular referrals to the Riverside University Health System - Behavioral Health, Preschool 0 - 5 Programs agency were asked by co-investigators (PCIT clinicians) in the study if they were interested in voluntary study participation. Families completed a standard assessment battery required by the county for their first session with the agency to determine which services were most appropriate (e.g., PCIT, Trauma-Focused Cognitive Behavior Therapy). Upon 
families' referral for PCIT services, the family was scheduled for an assessment session at which time the research project was explained by the clinicians administering treatment (i.e., IRBapproved research staff members), consent was read to the participants and obtained, and participants were provided a copy of their consent form. Eight PCIT-trained clinicians delivered treatment in the present study. Families were randomly assigned to either treatment group. Families were told which group they were assigned using specific wording detailed in a script. Participants were informed that they may withdraw from the research project at any time without penalty. After parents had consented, children were provided with a verbal description of the study. Children who were seven years or older received a copy of the written assent form, had the assent document explained to them, and then signed the document.

Data collection and knowledge of group placement began following the informed consent process. The family filled out the demographic form, followed by the ECBI, CBCL, and PSI:SF. During each of the following sessions, all families were recorded for their adherence to specified protocol including therapy attendance, session timeliness, completing assigned homework sheets, bringing the homework to session at least four times during the week (or $50 \%$ of the days between sessions) with audio recordings, and family achievement of meeting milestones (CDI mastery, PDI mastery, graduation).

The Riverside University Health System - Behavioral Health, Preschool 0 - 5 Programs agency contains a warehouse of gifted merchandise which they may freely distribute. Items in the warehouse vary over time and are dependent on what is gifted from distributers. Some merchandise previously in storage included purses, backpacks, flip flops, child pajamas, and brooms, for instance. For the incentive group, a family was given a random prize bag containing approximately $\$ 5-8$ in gifts (e.g., socks, slippers, hangers, shirts) upon on-time arrival and 
homework completion. This opportunity for a prize bag was made available at every scheduled therapy session the participant attended. Families were reminded using a standard script that they could receive prizes for each treatment session, and were told how they could go about obtaining those prizes (i.e., homework completion, timely attendance, mastery criteria). In addition, the participant was given another random prize bag when the family reached milestones including CDI mastery, PDI mastery, and PCIT graduation. The control group did not receive any compensation.

Prior to beginning the present investigation, the Riverside community mental health agency reached out to current families receiving PCIT who were not involved in the present study. The agency assessed the families' interest in receiving prizes representative of the items used in the study (5-point Likert scale; 1 = No Need for Item, 5 = Strong Need for Item). Feedback from families informed the research staff that the proposed value of incentives was appealing. All items that were ranked lower than an average score of 3 were not included as prizes in the study. Suggestions for each incentive prize were incorporated (e.g., size, quality) prior to initiating data collection.

Following the intake, the therapist recorded if the family attended the session and was on time, if the family attended but was late, if the family canceled, or if the family no-showed. The family then completed an ECBI. If the family was in the experimental condition (incentives), the family was given a mystery prize bag if they arrived on time. The therapist then checked to see if the family had completed over $50 \%$ of their homework on the homework sheet and audio recordings (4 out of 7 days) and recorded the number of days the homework was completed on the Session Record Form. If the family was in the experimental condition, the family was given a mystery prize bag if their homework was over $50 \%$ complete and recorded. If families failed to 
complete and bring their homework sheets to session, the family was required to complete the homework sheets during the checkout at the end of the session. After the homework sheet and recordings had been reviewed, the session continued according to the PCIT protocol (Eyberg \& Funderburk, 2011). Families were given a homework sheet to complete before the next session. For future sessions, if the family was in the experimental condition and reached mastery within the session (CDI, PDI) then they were given an additional mystery prize bag.

For the non-experimental group, the families followed the same protocol, but did not receive any form of incentives for their adherence to the protocol. For all families, data were deidentified, copied, and scanned to the primary investigator on a password protected Adobe document every week. These data were stored at West Virginia University’s Parent-Child Interaction Therapy's lab to be analyzed and interpreted.

Therapist assignment to experimental and comparison groups was assessed after $25 \%$, $50 \%$, and $75 \%$ of data collection. Data were assessed for patterns indicating a bias for group assignment to clinician or client success rates by clinician, but no patterns arose $(p$ 's $<.05)$. The incentive group contained more participants than the nonincentive group after $50 \%$ of data collection, therefore the primary investigator reviewed the protocol for enrolling families with the research staff. After a thorough evaluation of enrollment protocol by staff, it was determined that no problematic procedures were present. Therefore, brainstorming and discussion continued, but enrollment proceeded. Final enrollment displayed a continued disparity in treatment group numbers, but no concerns related to enrollment procedures remained.

\section{Results}

\section{Power Analysis and Randomization}

G*Power 3.1.9.2 was used to conduct a power analysis. Results revealed that 56 parentchild dyads would be adequate to achieve a power of .80 and a medium effect size (.25) with a 
repeated measures, within-between ANOVA. Researchers were able to collect a total of 84 families. Families were randomly assigned to groups with 50 families (59.5\%) enrolled in the incentive group and 34 families (40.5\%) enrolled in the nonincentive group.

\section{Preliminary Analyses}

To decrease the amount of missing data, researchers immediately asked participants if they intended to leave any of the items on a measure blank or if they would like to complete the missing items, when present. Preliminary analyses were conducted to evaluate the assumptions of the test, missing data points, and the normality of the data distributions. For all instances of incomplete items on a measure, directions for how to handle missing items were followed according to the measure's regulations (e.g., imputation based on the mean item score for the measure or subscale). Any measures missing over half of the responses would have been eliminated from analyses; however, this procedure was not needed in the present study unless a client had terminated from treatment and failed to complete measures at a given time point.

Preliminary analyses were run to determine if violations occurred in the areas of outliers, normality, linearity, and homoscedasticity. Normality was determined using the KolmogorovSmirnov Test and Q-Q plots. Linearity was determined using linear regression or Pearson correlations. Homoscedasticity was determined using Bartlett's test. Results from preliminary analyses are described throughout the results section below.

\section{Demographic Information}

Therapist demographic information included sex, age, ethnicity, language(s) spoken, education level, years as a clinician, and years delivering PCIT (Table 1). The nine clinicians participating in the study were $100 \%$ female, averaged 36.67 years of age $(S D=7.62)$, were largely Hispanic $(55.6 \% ; n=5)$, spoke English as a primary language $(88.9 \%)$, and spoke 
Spanish as a secondary language (44.4\%). Most had master's degrees (66.7\%), and all clinicians completed the PCIT online training, 40 hour in-person training, and 2-day advanced training. At the start of the project, the therapists averaged 8.33 years of clinical work $(S D=5.68)$ of which 4.89 years were spent conducting PCIT $(S D=3.95)$, had seen approximately 100 PCIT cases $(S D=93.13)$, and had graduated 44 of those PCIT cases $(S D=39.42)$

Data from the family demographic questionnaire were used to describe the overall sample as well as compare descriptive characteristics between groups for families with and without incentives. Researchers examined demographic variables including caregiver sex, age, ethnicity, yearly income, and reported psychopathology. Child demographics included age, sex, ethnicity, and reported psychopathology. Caregivers participating in the present study were $95.2 \%$ female ( $n=80)$, approximately 35 years of age $(M=35.28, S D=8.19)$, largely Hispanic $(63.1 \% ; n=$ 53), with approximately $31.0 \%$ indicating a history of mental health concerns (57.7\% depression, $42.3 \%$ anxiety, $19.2 \%$ trauma/domestic violence, $11.54 \%$ other). Children participating in the study were $66.7 \%$ male $(n=56), 3.75$ years of age $(S D=1.09)$, largely Hispanic $(60.7 \% ; n=$ 51), with $35.7 \%$ reporting previous mental health diagnoses (30.0\% autism spectrum disorder, $30.0 \%$ childhood disruptive disorder - not otherwise specified, $16.7 \%$ drug exposure, $13.3 \%$ attention-deficit/hyperactivity disorder, $23.3 \%$ other).

Chi-square and t-test analyses were conducted to determine if differences in any of these characteristics were present between groups (Tables $2 a-h$ ). Assumptions for both analyses were tested. Linearity, normality, homoscedasticity, and independence for t-tests were appropriate. All missing data were excluded from analyses. For family income, three outliers were detected and removed (Incentives: $n=2$; Nonincentives: $n=1$ ). In addition, income was also positively 
skewed and required a log transformation. Overall, no significant differences were found between groups across all variables ( $p$ 's $>.05)$.

\section{Aim 1}

Evaluate whether families referred to PCIT who were provided incentives for therapy had greater adherence to treatment components than families receiving PCIT as usual. Hypotheses and dependent variables associated with involvement are listed below:

A. It was predicted that families in the PCIT plus incentives group would require fewer sessions to obtain CDI mastery than families receiving PCIT as usual. Only families obtaining CDI mastery were included in this analysis $(n=46)$. The total number of sessions that families attended from the CDI Teach session to the CDI mastery session was the measured dependent variable.

All assumptions for the t-test were met (linearity, normality, homoscedasticity, independence). Twenty-five incentive families (50.0\%) and 21 nonincentive families (61.8\%) reached CDI mastery. No difference between the number of sessions families took to reach CDI mastery was found between the incentive group $(M=6.44, S D=3.04)$ and the nonincentive group $(M=6.23, S D=2.86), t(44)=.23, p=.82, d=.07$ (none; Table 3).

B. It was also hypothesized that families in the PCIT plus incentives group would need fewer weeks to obtain CDI mastery. The dependent variable was measured by the total number of weeks between the family's CDI Teach session and when the family reached CDI mastery. Comparisons were drawn from subtracting the date for CDI Teach from the date of CDI mastery. 
All assumptions for the t-test were evaluated. Data for this variable were positively skewed, therefore a square root transformation was conducted. All other assumptions were met. Twenty-five incentive families (50.0\%) and 21 nonincentive families $(61.8 \%)$ made it to CDI mastery. No difference between the length (in weeks) families took to reach CDI mastery was found between the incentive group $(M=13.10$, $S D=7.51)$ and the nonincentive group $(M=13.87, S D=6.98), t(44)=-.35, p=.73, d=$ .11 (none/small; Table 3).

C. It was expected that fewer families in the PCIT plus incentives group would drop out of treatment before reaching CDI mastery than families receiving PCIT as usual. Each family who dropped out before CDI mastery was tracked to determine if group differences were present for families able to obtain CDI mastery.

A chi-square analysis was utilized to evaluate this hypothesis. All assumptions for the chi-square analysis were met (sample size, categorical data, independence). No statistically significant associations between incentive group status and termination before CDI mastery were found, $\chi^{2}(1)=1.54, p=.21, \phi=.14$ (small; see Table 4).

Hypotheses D - G all relate to the larger hypothesis that families receiving incentives would have greater adherence to treatment components than families not receiving incentives. Each of the following hypotheses pertains to specific treatment components; as such, all hypotheses are first outlined followed by the results for all hypotheses.

D. For the first hypothesis, it was predicted that families in the PCIT plus incentives group would have a smaller percentage of no-shows to scheduled therapy sessions than families receiving PCIT as usual. The total number of no-show sessions was divided by the total 
number of scheduled sessions for a family. The percentages were compared across groups.

E. Similarly, families in the PCIT plus incentives group were expected to have a smaller percentage of cancellations for scheduled therapy sessions than families receiving PCIT as usual. The total number of cancelled sessions was divided by the total number of scheduled sessions for a family.

F. Additionally, families in the PCIT plus incentives group were expected to have a smaller percentage of sessions in which they were late (10 minutes or more) than families receiving PCIT as usual.

G. Lastly, families in the PCIT plus incentives group were hypothesized to have a greater amount of homework assignments completed (both paper and audio recordings) than families receiving PCIT as usual. Audio recordings and homework sheets were required to be completed for CDI homework at least 4 times out of 7 days during the week. Homework sheets were also required to be completed using the same guidelines for PDI homework, but audio recordings were not necessary during the PDI rollout. If families had a longer or shorter break between weekly PCIT sessions, over 50\% of the daily homework had to be completed for the entire duration between the previous and most recent PCIT session. The average amount of audio and paper homework completed per week was recorded for each family and compared between groups.

To analyze the preceding hypotheses, t-tests were run for each variable. Due to the higher probably of having Type 1 error with multiple t-tests, Bonferroni corrections were used when the outcomes were examined. Since five variables were analyzed (and 
the standard $\alpha=.05)$, outcomes for each analysis needed to be significant at the .01 level $(\alpha=.05 / 5=.01)$

All assumptions for the t-test were evaluated. Data for all of the five variables (no-shows, cancellations, late attendance, paper homework, and audio homework) were positively skewed. All variables underwent a square root transformation, except late sessions which required a log transformation. All other assumptions were met.

Across the assessed variables, no significant differences arose (Table 5).

Specifically, there were no significant differences in the percentage of no-shows between the incentive $(n=50 ; M=.11, S D=.15)$ and nonincentive group $(n=34 ; M=.16, S D=$ $.18), t(82)=-1.79, p=.08, d=.41$ (small/medium). Next, no statistically significant difference in the percentage of cancellations between the incentive group $(n=50 ; M=$ $.25, S D=.18)$ and nonincentive group $(n=34 ; M=.21, S D=.15)$ were found, $t(82)=$ $1.01, p=.31, d=.19$ (small). Analyses of percentage of late sessions were also found to have no statistical significant difference between the incentive group ( $n=50 ; M=.04$, $S D=.06)$ and nonincentive group $(n=34 ; M=.06, S D=.14), t(82)=-1.31, p=.20, d=$ .18 (small).

For homework completion, neither paper homework $(t(82)=-.69, p=.49, d=$ .15 ; none/small) nor audio homework $(t(82)=-.02, p=.99, d=.00$; none) yielded statistically significant differences in average weekly rates of completion between the incentive and nonincentive groups. Both groups only completed approximately $1-2$ paper homework assignments per week (Incentives: $M=1.69, S D=1.67$; Nonincentives: $M=2.01, S D=1.91$, while only about 1 audio homework assignment was completed per 
week, when required (Incentives: $M=1.15, S D=1.37$; Nonincentives: $M=1.14, S D=$ $1.52)$.

\section{$\operatorname{Aim} 2$}

Evaluate whether families referred to PCIT who were provided incentives for therapy had greater success in treatment than families receiving PCIT as usual. Hypotheses and dependent variables associated with treatment success are as follows:

A. It was predicted that families in the PCIT plus incentives group would have a greater number of families attain CDI mastery than families receiving PCIT as usual. Therapists reported on attainment of CDI mastery using the Session Record Form for each session. The total number of families attaining CDI mastery were recorded and compared for each group. CDI mastery was defined as obtaining 10 behavior descriptions, 10 labeled praises, and 10 reflections with less than 3 total questions, commands, or negative talks in a 5-minute coded DPICS interaction.

A chi-square analysis was run to test this hypothesis. All assumptions for the chisquare analysis were met. No statistically significant associations between incentive group status and attainment of CDI mastery were found, $\chi(1)=1.54, p=.21, \phi=.14$ (small; see Table 6).

B. It was expected that families in the PCIT plus incentives group would have a greater number of families attain PDI mastery than families receiving PCIT as usual. Therapists reported on attainment of PDI mastery using the Session Record Form for each session. The total number of families attaining PDI mastery was recorded and compared for each group. PDI mastery was defined as obtaining $75 \%$ effective commands and $75 \%$ effective follow-through in a 5-minute coded DPICS interaction. 
Another chi-square analysis was run to test PDI mastery attainment. All assumptions for the analysis were met. No statistically significant difference between groups was found for achieving PDI mastery, $\chi(1)=0.31, p=.58, \phi=.06$ (none/small; see Table 7).

C. Families in the PCIT plus incentives group were expected to have a greater number of families graduating from PCIT than families receiving PCIT as usual. Therapists reported on attainment of graduation using the Session Record Form for each session. The total number of families who attained graduation were recorded and compared for each group. Graduation was defined as making it to the final graduation session after reaching CDI mastery, PDI mastery, and PDI roll out.

A final chi-square test was run to determine if differences between groups arose for attainment of PCIT graduation. All assumptions for the chi-square test were met. All families who attained PDI mastery went on to make it to PCIT graduation. Therefore, no differences between groups were found for PCIT graduation, $\chi(1)=0.31, p=.58, \phi=.06$ (none/small; see again, Table 7).

D. It was hypothesized that families in the PCIT plus incentives group would have a greater decrease in child behavior problems as measured by the Intensity scale of the Eyberg Child Behavior Inventory (Eyberg \& Pincus, 1999) than families receiving PCIT as usual. T-scores were used to draw standardized comparisons between groups. Comparisons were made using the first ECBI score and the ECBI score for each family's last recorded session.

A repeated $2 \times 2$ ANOVA was conducted to test this hypothesis. All ANOVA assumptions were met (independence, normality, homoscedasticity). A within-group 
analysis revealed a significant main effect in ECBI Intensity scores for treatment, $F(1$, $81)=59.40, p<.01, \eta^{2}=.42$ (large). However, the between-group analysis found no statistically significant interaction effect, $F(1,81)=.38, p=.54, \eta^{2}=.00$ (none). Cell sizes, means, and standard deviations for the between-group factorial design are provided in Table $8 \mathrm{a}$ for T-scores and Table $8 \mathrm{~b}$ for raw scores. Visual representation of T-score comparisons between groups can be found in Figure 1.

E. It was expected that families in the PCIT plus incentives group would have a greater decrease in internalizing and externalizing behavior problems as measured by the Externalizing and Internalizing scales of the Child Behavior Checklist (Achenbach \& Rescorla, 2001) than families receiving PCIT as usual. Raw scores were used (due to limitations of T-score reporting by the community agency) to draw standardized comparisons between groups at pretreatment, midtreatment, and posttreatment.

Two $2 \times 3$ level repeated measures ANOVAs were conducted to test the assumption that externalizing and internalizing symptoms would improve more for incentive families than nonincentive families. Due to a limited number of families reaching graduation, a total $n$ of 31 was included (Incentive group: $n=17$; Nonincentive group: $n=14)$. Analyses were run with low power (.35) due to the small sample size. All assumptions for the Externalizing scale were met (e.g., Mauchly's Test of Sphericity and Levene's Test, $p$ 's $>.05)$. The within-group analysis revealed a significant main effect in CBCL Externalizing scores for treatment, $F(2,28)=39.24, p<.001, \eta^{2}=.57$ (large). The CBCL Externalizing scale by incentive group found no statistically significant interaction effect, $F(2,28)=5.54, p=.90, \eta^{2}=.03$ (small). Cell sizes, means, and standard 
deviations for the factorial design are provided in Table 9. Figure 2 contains betweengroup comparisons.

All assumptions for the Internalizing scale were also met. A statistically significant main effect was found for CBCL Internalizing scores for treatment, $F(2,28)=$ 13.56, $p<.001, \eta^{2}=.30$ (large). No statistically significant interaction effect for the CBCL Internalizing scale by incentive group was found however, $F(2,28)=.002, p=$ $1.00, \eta^{2}=.04$ (small). Cell sizes, means, and standard deviations for the factorial design are provided in Table 10. For visual group comparisons, see Figure 3.

F. Lastly, it was hypothesized that families in the PCIT plus incentives group would have a greater decrease in parenting stress levels as measured by the Total Stress score on the Parenting Stress Index: Short Form (Abidin, 1990) than families receiving PCIT as usual. Again, raw scores were used (due to limitations of T-score reporting by the community agency) to evaluate between-group differences at pretreatment, midtreatment, and posttreatment.

A 2 x 3 level repeated measures ANOVA was run to determine if parenting stress varied over time between incentive families than nonincentive families. All assumptions for the Total Stress scale were met. Within-group comparisons yielded a significant main effect for PSI:SF Total Stress scores over the course of PCIT, $F(2,28)=26.35, p<.001$, $\eta^{2}=.51$ (large). Between-group comparisons found no statistically significant interaction effect (PSI:SF Total Stress x Group), $F(2,28)=1.09, p=.35, \eta^{2}=.04$ (small). Cell sizes, means, and standard deviations for the factorial design are provided in Table 11. Figure 4 contains between-group comparisons.

\section{$\operatorname{Aim} 3$}


A cost analysis was conducted to determine if there was any financial difference between the PCIT plus incentives group and the PCIT as usual group in the community mental health agency.

In determining whether PCIT with incentives could be a sounder option for community mental health agencies, all costs incurred by the agency for irregular attendance and cost of incentives was subtracted from the total potential income of the clients scheduled hours. Calculations for cost analysis are based on guidelines from Drummond and colleagues for economic evaluations of health care programs (Drummond et al., 2005). The cost analysis equation for this study is as follows:

Cost analysis $=\{(\#$ attended sessions $) X$ (Hourly rate of agency $)\}-\{(\#$ cancelled sessions + \#no shows) X (Hourly rate of agency)\}(Number of prizes received $X$ average cost of prize)

Each family's cost analysis was calculated according to the defined equation. Means for both the incentives and nonincentives groups were also calculated. Group differences were assessed through a t-test.

The agency reported using Medicaid for payment from clients (no private insurance utilized). Billing rates for Medicaid were reported as $\$ 2.69$ per minute. PCIT sessions were blocked for 90 minute periods, therefore sessions were calculated as being worth $\$ 242.10$. Total attended sessions, missed sessions (cancels and no-shows), and total number of prizes received by a family were calculated. Attended sessions and missed sessions were both multiplied by the session rate $(\$ 242.10)$, and the average cost of an incentive (\$6.50) was multiplied by the number of prizes a family received. The total cost lost from client misses (missed x session cost) and prizes (total prizes $\mathrm{x}$ average cost of prizes) was subtracted from the total profit gained from clients (attended x session cost). 
Assumptions for t-tests were explored and were met. Means, standard deviations, cell sizes, t-test outcomes, and Cohen's d are reported on Table 12. No differences were found in the cost analysis outcomes between groups, $t(82)=-.29, p=.78, d=.06$ (none). The average revenue made by the agency per family who attended PCIT sessions ranged from approximately $\$ 1,500-$ $\$ 1,600$ over the course of treatment in each group.

\section{Exploratory Question}

The researchers were interested in determining which variables were most highly associated with attrition from PCIT.

Binary logistic regression. First, variables related to attrition for both groups in the study were examined via a binary logistic regression. Relations between dropout from PCIT and other factors shown to be predictive of early termination in the literature including caregiver age, ethnicity, yearly income, psychopathology, stress (PSI:SF Total Stress score), parental verbalizations (DPICS positive composite-labeled praise, behavior description, reflection; negative composite - negative talk, command, question), and severity of child behavior problems (CBCL Internalizing and Externalizing scales) were explored. In addition, analyses explored if group assignment (incentives or no incentives) would account for a significant amount of the variance beyond the previously listed variables found to associate with early termination.

All assumptions for the logistic regression were met. The effects of incentive group status; caregiver age, income, ethnicity, and mental health history; parenting stress; positive and negative parental verbalizations; and internalizing and externalizing child behaviors were explored to determine how they uniquely contributed to the likelihood that families would terminate early from treatment. The model was statistically significant $\chi^{2}(12)=23.08, p=.027$. It explained $39.7 \%$ (Nagelkerke $R^{2}$ ) of the variance in termination status and correctly classified 
$80.3 \%$ of the cases. Only two variables were statistically significant in the regression including caregivers with "mixed" ethnicities and children presenting with more severe internalizing behaviors. Specifically, parents with mixed ethnicity were found to be 43.52 times more likely to terminate than Hispanic caregivers. Hispanic caregivers were chosen as the reference group due to this being the highest number of families enrolled. Also, children with greater internalizing symptoms were found to be 1.17 times more likely to terminate treatment than children without as severe internalizing symptoms. Outcomes for this analysis can be found in Table 13.

TAI \& BTPS. To further analyze additional factors that may limit families' ability or desire to come into treatment, families completed a measure on their perceptions of barriers to treatment using the BTPS and on their satisfaction with treatment using the TAI. Descriptive data were pulled to analyze what factors presented the greatest challenges for each family.

Comparing the current sample to a standardized sample. Barriers to treatment participation were examined for this sample and compared to a standardized sample of children and families receiving outpatient services in the literature (Kazdin, Holland, Crowley, \& Breton, 1997). A total score from the BTPS was calculated by summing all measure items for each family at CDI Coach 3 time point, descriptives were collected (i.e., $n, M, S D$ ), and then a t-test comparing these data to a standard sample was conducted. Descriptive data found a mean rating of $57.80(S D=8.95)$ for 59 families. A study including families of children in outpatient treatment was used as the comparison sample (Kazdin, Holland, Crowley, \& Breton, 1997). The descriptive statistics for the sample study were as follows: $n=260, M=65.97, S D=13.83$. A ttest was conducted with the two samples. Outcomes indicated the present study sample having significantly less barriers to treatment than the standardized sample from the literature, $t(317)=$ $4.33, p<.001, d=.70$. 
General outcomes. General outcomes were explored for all participants regardless of incentive group status $(N=84)$ at CDI Coach 3 (the earliest point of measurement for these assessments) using descriptive statistics. For the TAI, since scores closer to 1 indicate problems or worsening of issues and scores closer to 5 indicate high satisfaction or improvement, only scores with means at 3 or below are reported. However, no ratings for any of the TAI's items fell below a mean of $3.84(S D=.56$; Item $=$ new child behavior problems since starting treatment $)$. Next, descriptive statistics for items on BTPS at CDI Coach 3 were explored. Scores on the BTPS closer to 1 indicate that families did not find the items an issue in coming to treatment while scores closer to 5 were rated as very often a problem for families; therefore, only items rated 3 or above are reported. Again, no ratings for any of the BTPS's items fell above a mean of $2.47(S D=1.41 ;$ Item $=$ high family stress $)$. These same descriptive statistics were explored at midtreatment. No items were reported by families as either occasionally or often problematic on the TAI or BTPS (scores $\geq 3$, or $\leq 3$, respectively) at midtreatment measurements.

Outcomes by incentive group status. Scores on both the TAI and BTPS were tallied for time points at CDI Coach 3, midtreatment (after attaining CDI mastery), and posttreatment (at graduation). Differences between incentive and nonincentive group means were explored using independent sample t-tests. All assumptions for the t-tests were met. No differences were found between incentive and nonincentive groups at CDI 3, midtreatment, or posttreatment for scores on the TAI Total scale, BTPS Competing Activities/Life Stressors, BTPS Relevance of Treatment, BTPS Relationship with Therapist, or BTPS Treatment Issues subscales ( $p$ 's > .05).

Outcomes by CDI mastery status. Next, exploratory t-tests were run to determine if any particular items or scales at the CDI Coach 3 session for the TAI or BTPS were significantly different for families terminating before or attaining CDI mastery status. All t-test assumptions 
were explored and met for the TAI and BTPS scales. No differences were found between families who attained CDI mastery status and those who did not for CDI Coach 3 measures on the TAI Total scale, BTPS Competing Activities/Life Stressors, BTPS Relevance of Treatment, BTPS Relationship with Therapist, or BTPS Treatment Issues subscales ( $p$ ’s > .05).

Individual items for both the TAI and BTPS were then explored. Twenty eight of the 44 items from the BTPS were positively skewed requiring either square root or log transformation corrections. Remaining assumptions were met unless otherwise specified. No differences were found between groups in t-tests for TAI CDI 3 scores between families who did and did not attain CDI mastery $(p ' s>.05)$. All of the following variables had a significant Levene's tests and were corrected; for the BTPS, child session refusal $(t(41)=2.56, p=.01, d=$ could not be calculated), child development of new or different problems $(t(56.81)=2.89, p<.01, d=.69$; medium/large), and caregiver disagreement with his/her partner about coming to treatment at all $(t(41)=2.65, p=.01, d=$ could not be calculated) were all found to be statistically significantly different for families terminating treatment prior to CDI mastery and families attaining CDI mastery (Table 14). The remaining 41 BTPS items were nonsignificant ( $p$ ’s > .05).

Outcomes by PCIT graduation status. Exploratory t-tests were also run to determine if any items at the CDI Coach 3 session or at the midtreatment session (after attaining CDI mastery) on the TAI or BTPS were statistically significantly different for families terminating treatment before or reaching PCIT graduation status. All assumptions for t-tests were tested and met for the TAI and BTPS scales. No differences were found between families who attained graduation status and those who did not for both CDI Coach 3 and midtreatment measures on the TAI Total scale, BTPS Competing Activities/Life Stressors, BTPS Relevance of Treatment, BTPS Relationship with Therapist, or BTPS Treatment Issues subscales ( $p$ ’s > .05). 
Individual items for both the TAI and BTPS were then explored. In addition to the 28 items for BTPS from CDI Coach 3 that required a transformation, 26 of the 44 items from the BTPS required square root or log transformations at the midtreatment time point. No items from either the TAI or the BTPS were statistically significantly different between families who graduated and those who did not at CDI Coach 3 assessment. At midtreatment, no items on the TAI were significantly different between graduators and terminators, but BTPS's item for parental sickness limiting treatment attendance (all assumptions met) was significantly different between groups, $t(39)=-3.57, p<.01, d=1.22$ (large; Table 15). No other of the 43 BTPS items were statistically significant ( $p$ 's $>.05)$.

Outcomes by ethnicity. Final analyses were conducted to explore if differences were present between families identifying as Hispanic compared to mixed ethnicities.

CDI mastery and graduation status. Chi-square analyses were run to compare if any differences arose in CDI mastery or graduation attainment between Hispanic and non-Hispanic families. All assumptions of the chi-square tests were met. No differences were found between Hispanic and non-Hispanic families in attaining CDI mastery, $\chi(3)=0.75, p=.86, \phi=.09$ (none/small; Table 16). Additionally, no differences were found between these groups in reaching PCIT graduation, $\chi(3)=2.48, p=.48, \phi=.17$ (small; see Table 17).

Child behavior problems. Differences between Hispanic and non-Hispanic families' ratings of child behavior problems, as measured by the ECBI Intensity scale, were compared using a repeated measures, within-between 2 x 2 ANOVA. All ANOVA assumptions were met. A within-group analysis revealed a significant main effect in ECBI Intensity scores for treatment, $F(1,81)=20.06, p<.001, \eta^{2}=.20$ (medium/large). The between-group analysis interaction effect was not statistically significant, $F(1,81)=.70, p=.56, \eta^{2}=.00$ (none). Cell sizes, means, 
and standard deviations for the between-group factorial design are provided in Table 18a for Tscores and Table 18b for raw scores. Visual representation of T-score comparisons between groups can be found in Figure 5.

TAI \& BTPS. T-tests were conducted to determine if any differences arose at CDI Coach 3 TAI and BTPS assessments between Hispanic and non-Hispanic families (i.e., Caucasian, African American, mixed) on both individual items as well as scales. All assumptions were explored for the t-tests. Twenty eight of the 44 items from the BTPS were positively skewed requiring either square root or log transformation corrections. Remaining assumptions were met unless otherwise specified. Outcomes found that Hispanic families reported significantly greater positive ratings of TAI's item on "treatment helped with other problems," $t(58)=3.12, p<.01, d$ $=.81$ (large). Hispanic families also reported significantly more issues the BTPS item on child session refusal $(t(36)=2.58, p=.01, d=$ could not be calculated $)$. However, Hispanic families had significantly less concerns related to experiencing high levels of stress $(t(57)=-2.08, p=$ $.04, d=.55$; medium), losing interest in coming to treatment $(t(21)=-2.82, p=.04, d=$ could not be calculated), as well as the BTPS scale Competing Activities/Life Stressors $(t(57)=-2.25, p=$ $.03, d=.58$; medium) than non-Hispanic families (Table 19). No other TAI or BTPS items or scales were significantly different between the two groups.

\section{Discussion}

The current study explored a randomized controlled trial in a community mental health agency for a largely Hispanic and low income population. Specifically, families were randomly assigned to receive incentives for adhering to components of Parent-Child Interaction Therapy including session attendance, homework completion, and mastery attainment of therapeutic skills (i.e., CDI, PDI, graduation). The following factors were selected based on evidence supporting 
their associations with positive client outcomes; namely, attendance and graduation were found to be associated with maintenance of therapeutic skills (Eyberg et al., 2008), homework use has been shown to enhance outcomes of treatment in general (Kazantzis et al., 2010; Mausbach et al., 2010), and failure to reach mastery or graduation has been associated with continued child behavior problems and parental stress (Boggs et al., 2004). The purpose of the present study was to evaluate if implementing incentives was an effective method for improving core concerns related to child mental health services.

Outcomes from this study indicated null results when comparing families who received incentives to those who received treatment as usual across a number of research questions including the following: time to reach CDI mastery (number of sessions, weeks in treatment); number of families terminating from treatment; rate of treatment session no-shows, cancellations, late sessions, or homework completion (i.e., paper, audio); number of families reaching mastery in CDI, PDI, and graduation; changes in child behavior problems (ECBI Intensity scale, CBCL Internalizing and Externalizing scales) and parenting stress (PSI:SF); and outcomes of cost analysis. Exploratory analyses indicated significant family barriers for families terminating from treatment related to parental sickness, partner disagreements with attending treatment, child session refusal, and change in child behavior problems. Insight into these findings and future directions about reducing family attrition through innovative new approaches are discussed below.

\section{Incentives}

The present study tested a novel approach to improve a systemic problem in Parent-Child Interaction Therapy and child mental health services, more generally (Lanier et al., 2011; NIMH, 2001). Specifically, incentives were provided to families in an effort to increase attendance, 
homework completion, and mastery of therapeutic skills. Based on previous findings noted in the substance abuse literature on positive behavior change (e.g., Petry et al., 2004; Silverman et al., 1999), it was postulated that incentives may provide a viable option for increasing important client behaviors in parent training therapy (i.e., PCIT).

Although substance abuse research has found some success in their patient populations for higher rates of adherence to treatment goals (e.g., Petry et al., 2004; Pollastri et al., 2005), there are a number of reasons why the current study may have been ineffective in changing family behavior in PCIT. First, incentives used in substance abuse research are frequently large (Petry et al., 2004). Similar to a lottery system, many patients are given the opportunity to win thousands of dollars for continued treatment adherence over time. Using expensive incentives has been justified in the literature due to the savings gained from associated health and social costs (e.g., fewer drug-related crimes or overdoses, greater workplace productivity; NIDA, 2018). Researchers and funders have touted treatment incentives as cost effective alternatives to incarceration $(\$ 24,000 /$ year per person), therefore allowing the cost of treatments such as methadone maintenance $(\$ 4,700 /$ year per person) to be within reasonable ranges (NIDA, 2018). While incentives were provided immediately and more frequently to families in the present study (dependent on client adherence), the incentives for the current project were well below the range often found in substance abuse research (with prizes ranging from $\$ 5-8$ ). Studies show that effectiveness of incentives can be lost if the incentive is too low given the treatment goal and the focus population (Silverman et al., 1999). The present study may have limited effects due to rewards being below the threshold for incentivizing client behavior change.

While many studies looking at the effective implementation of incentives to reduce substance abuse problem behaviors have high rewards, there have been a few programs which 
utilized low cost incentives with some success. For example, Pollastri and colleagues (2005) provided vouchers for goods and services for as little as $\$ 3$ which were found to positively impact participants' unanticipated no-shows to treatment sessions. Importantly however, participants also had the opportunity to earn take-home methadone doses which may have been highly motivating given the individuals' present drug addictions. Moreover, it is possible that these substance abusers (i.e., cocaine and opioid) may have allocated the remaining resources they had to their drug habit. The presence of small vouchers for necessities (e.g., food, clothes) may have had a greater impact on their livelihood and been more incentivizing when compared to the present study's sample. Although families receiving incentives in PCIT from this study generally had low incomes, families may still have had enough resources to afford goods that were provided as incentives (e.g., child clothing, school supplies) therefore limiting their incentivizing value.

Another reason incentives may not have translated from substance abuse studies to the present study is that low cost incentives may not often work for drug dependent populations either. It is possible that literature on substance abuse only highlights the few treatments which are effective. In general, rates of relapse are high in this substance abuse population and many treatments are found to be ineffective in combatting drug addictions (see Donovan, 2003, for a review). Since treatments may frequently fail to produce successful outcomes for those battling substance abuse (Donovan, 2003), it is possible that studies using low cost treatments are not frequently published due to their lack of findings. Those that have been published may be the exceptions rather than common practice. Due to the possible low rate of success with low cost options, the field has instead shifted to promoting high cost treatments to get a greater likelihood for individuals' success (e.g., long-term [6 - 12 month] residential treatments, 
pharmacotherapies, motivational incentives [around \$100 prizes]; NIDA, 2018). This level of costs may be necessary to also elicit effective outcomes from families attending parent training; however, appropriate cost effectiveness studies would need to be conducted prior to justifying these expenditures.

Only a few studies have been conducted to test incentives outside of substance abuse populations. Although some projects have shown success while using incentives to increase family adherence or child behaviors (Eyberg \& Johnson, 1974; Frye, 2011; Hayes et al., 2000), additional studies have yielded null results similar to the current project (e.g., academic achievement, attendance at treatment for depressed mothers, attendance at a parent training program; Dumas et al., 2010; Frye, 2011; Heinrichs \& Jensen-Doss, 2010; Sacks et al., 2015). Studies that have found success with utilizing incentives differed from our study in a number of ways. For example, Eyberg and Johnson's (1974) study recruited families who were willing to pay for $12-24$ sessions of treatment before initiating therapy and also eliminated families who prematurely terminated from treatment. Both of these methods contributed to the overestimation of their treatment's effects due to the selection bias of families and their subsequent outcomes (e.g., selecting families with higher socioeconomic status). As another example, the Hayes and colleagues' (2000) study which required families to sign a contract to attend four consecutive appointments before receiving a \$30 coupon had only three enrolled families and provided a short duration of treatment (four sessions vs. unknown number of sessions before attaining mastery criteria) to receive the incentive. In turn, the literature has shown that a large number of families drop out after four sessions of family therapy (Liebsack, 2016; McKay, Harrison, Gonzáles, Kim, \& Quintana, 2002); it is possible that the \$30 coupon and signed contract 
provided an additional incentive to reach four sessions, but many families typically attend at least four sessions regardless of incentives.

Irrespective of the differences, a common issue raised regarding the use of incentives outside of drug-based programs is the significant level of barriers families experience that prevent involvement in family therapy over time (Kazdin, Holland, Crowley, \& Breton, 1997). For example, in Sacks and colleagues' (2015) study of pregnant mothers with depression who received incentives for attending psychological services, it was posited that these mothers failed to attend treatment due to a lack of childcare for their other children. In turn, our sample of parents (largely mothers) also reported some difficulties with parental sickness preventing the families from coming to treatment compared to families who reached PCIT graduation. Upon further discussion with the clinical supervisor of the team in Riverside, the researcher found that some families who terminated from treatment gave birth or had pregnancy complications that prevented the continuation of PCIT. Anecdotally, one mother reported wanting to get her child's behavior under control prior to the birth of her next child. Since PCIT studies are largely conducted with mothers, as was the case in the present sample (e.g., Agazzi, Tan, Ogg, Armstrong, \& Kirby, 2017; Harwood \& Eyberg, 2006; Hood \& Eyberg, 2003), one barrier to consider when implementing PCIT in general is that some families may be expanding during the time family therapy is occurring. Although parental motivation to come to treatment may be high, barriers such as the arrival of a new baby or a complicated pregnancy can prevent families from continuing treatment.

Other research conducted with parent training courses have found that incentives may have a strong impact on enrolling vulnerable families with limited levels of education (Dumas et al., 2010; Guyll, Spoth, \& Redmond, 2003) who may not otherwise participate in parent training 
programs (Heinrichs, 2006). Unfortunately, as was the case in the present study, the use of these incentives has not been effective in maintaining families in treatment. Additional stressors or barriers to treatment may play a role in regular attendance for these families over time.

Within the context of cognitive evaluation theory, concerns have been raised about the possible diminishment of intrinsic motivation when extrinsically motivating rewards are implemented (Deci \& Ryan, 1980). Intrinsic motivation refers to self-determination and regulation while extrinsic motivation refers to causes of behavior outside of the individual (Cheng \& Yeh, 2009). It has been argued that implementing extrinsically motivating rewards comes with the risk of removing intrinsic motivation and negatively impacting treatment outcomes otherwise seen by the gains made in effective treatments (Kohn, 1993). Some researchers claim that implementing incentives temporarily changes behavior, but does not create lasting effects (Deci, Koestner, \& Ryan, 2001; Kohn, 1993). In turn, if the intrinsic motivation of improving the parent-child relationship is removed (and replaced with externally-motivating incentives), then any changes in parent behaviors are only maintained due to incentives, and the improvements would be nonexistent when incentives were removed. While it is possible that the incentive group in the present study was ineffective because half of the sample increased engagement while the other half of the sample lost intrinsic motivation, the incentivized behavior of adherence components was not found to be different between groups. In addition, both groups demonstrated significant within-group changes related to child behavior problems and parenting stress. This investigation, therefore, provided no evidence that incentive status was associated with any iatrogenic changes in intrinsic family motivation or the effectiveness of PCIT on measured variables (Eisenberger \& Cameron, 1996; Heinrichs \& Jensen-Doss, 2010). Yet, 
philosophical concerns about the possible loss of intrinsic motivation when using incentives for parent training should be examined more systematically in any future research in this area.

Another possible explanation for the lack of effectiveness of incentives in this study is that the prizes were not desirable to the families, and therefore did not serve as reinforcement. Anticipating this possibility, precautions were taken by study personnel to examine the interest garnered by the potential prizes prior to distributing them as reinforcers for adhering to PCIT treatment components. Importantly, the agency collected information from a sample of their client base on their feelings toward and preferences for possible incentives. The agency explored client preference to ensure future enrolled families would find the prizes desirable and would be interested in receiving them to increase the probability that the prizes would serve as a reinforcer. Outcomes indicated that families from this population viewed the receipt of prizes favorably. In turn, items that were rated as less desirable by families were removed as options for incentives prior to starting study enrollment.

An alternative option for incentives having no impact on family behavior was the way prizes were delivered. Specifically, it is possible that monetary rewards, gift cards, or a token economy in which families could select their prizes from a clinic-based store would have been options that were more reinforcing than the incentives in the present study. Studies using monetary incentives have been found to significantly impact such behaviors as medication adherence in patients with psychotic disorders (€30 per month for full compliance; Noordraven et al., 2017) or session attendance in family therapy ( $\$ 30$ coupon for four sessions; Hayes et al., 2000). Gift cards have also been found to be successful in reducing unanticipated appointment no-shows in substance abusers (Pollastri et al., 2005) as well as increasing attendance in telephone-based monitoring programs for substance use disorders ( $\$ 10$ per session; Van Horn et 
al., 2011). The use of monetary prizes or gift cards may have allowed participants to buy what they wanted or needed and increased the desirability of the incentive. Although a store-based option could not be found in the literature, it is possible that participants may have found greater reinforcement from selecting their own prizes than having clinicians determine the prizes for them. Individual client preference assessments for incentive prizes may have increased the likelihood of providing incentives that acted as reinforcers. In addition, these preference assessments for each client would have provided valuable insight into the impact of the incentives on participating families' behaviors.

Unfortunately, the agency did not explore if prizes were perceived differently among families with different ethnicities (as the present sample was largely Hispanic); however, the agency ensured the sample was representative of their usual client base. To evaluate the possibility of cultural issues in the perception of prizes for performance, a literature review was conducted. The only research discovered by this review pertained to cultural indicators related to gift exchanges when Hispanic families were the ones giving the items (Martínez-Taboas et al., 2014; Vasquez, 2007). Given the lack of data and literature in the area, it is unknown whether the ethnicity of this sample affected the results. While the prizes produced no meaningful change in family behavior over time, families in the pilot did generally report finding the available prizes to be desirable. Yet, because individual preference assessments were not conducted and families were not given choices with regard to the incentives, the possibility remains that particular families may not have found certain prizes to be desirable, thereby decreasing the reinforcing value of the incentives on an individual level.

\section{Cultural Concerns}


Although it was not exclusively targeted, the research team would be remiss if the outcomes of the present study were not discussed in relation to the participants' demographics and culture. With the majority of caregivers and children in this study identifying as ethnically Hispanic (Padilla, 2002), this is one of the few projects in PCIT that has evaluated a minority and Hispanic population (e.g., Barnett et al., 2017; Niec et al., 2014; Stokes et al., 2016) and one of even fewer randomized controlled trials (McCabe \& Yeh, 2009). Despite having many evidencebased treatments normed for Caucasian or European American populations, a dearth of work has been done to adapt treatments and disseminate them to Hispanic populations (Cardona et al., 2012; Kaminski, Valle, Filene, \& Boyle, 2008). Previous research on PCIT with this population points to effective outcomes for Spanish-speaking Mexican-American families when PCIT is largely unmodified (Borrego, Anhalt, Terao, Vargas, \& Urquiza, 2006). Similar outcomes were found in the present study as no differences in CDI mastery and graduation attainment as well as change in child behavior problems (ECBI Intensity scale) were found between Hispanic and nonHispanic families. However, some tailoring is still recommended to increase treatment success. In line with Borrego's work (2006), clinicians in the present study delivered treatment in Spanish to families who identified Spanish as their primary language. In addition, measures translated into Spanish were available for families who preferred non-English versions.

Importantly, the measure used to assess barriers in treatment (Barriers to Treatment Participation Scale) has not yet been standardized with a Hispanic population (A. Kazdin, personal communication, March 22, 2016). Although great lengths were taken in the present study to translate measures such as the BTPS to accommodate families who may not be literate in English, it is possible that the measure did not accurately assess items in the same manner due to inherent differences between the cultures or in the translated terms (McCabe, Yeh, Garland, 
Lau, \& Chavez, 2005). Previous research has shown that Hispanic families benefit less from mental health treatment due to practical barriers (e.g., less likely to access mental health treatment, transportation), cultural barriers (e.g., discouragement from family members, stigma of mental health treatment), acculturation (e.g., unfamiliarity with mental health system), and beliefs about problems related to receiving mental health treatment (e.g., problems handled within the family, associating temperament with child behavior; McCabe et al., 2005). When compared to non-Hispanic families in the current study, Hispanic families were less likely to feel high levels of stress, less likely to have low interest in coming to treatment, and less likely to have numerous competing activities; Hispanic families did report more difficulties with child session refusal, yet had higher beliefs that treatment was helpful with more than the original reported concerns (when compared to non-Hispanic families). Interestingly, the unique factors distinguishing Hispanic and non-Hispanic families in the present study pointed to generally similar experiences with barriers. However, the areas which differed between these groups pointed to Hispanic families having fewer barriers and more positive views of treatment. Therefore, it is possible that although families from Hispanic backgrounds reported fewer barriers, the ones they did experience had greater impact on their ability to attend treatment. Future research should explore the effect of ethnicity on reporting of barriers in treatment to determine additional factors that may contribute to participation in family therapy over time. Further adaptations outside of the realm of the present study that have been integrated into PCIT for Hispanic families include cultural assessments to inform individualization of treatment, describing treatment as "educational," allowing additional time for rapport-building between therapists and caregivers, including more examples of Hispanic families into training components, and incorporating important family members besides the mother (McCabe et al., 
2005). While these various components could have each been incorporated in some capacity depending on a therapist's skills or cultural awareness, these factors were neither tracked nor measured for fidelity across the present study. PCIT has been shown to be a robust treatment across a number of diverse families (for a review, see Butler \& Eyberg, 2006; Werba et al., 2006), and the present study found that families were successful at similar rates across ethnicity status. Still, further adaptations for Hispanic families may have increased the positive outcomes above and beyond the other families in this study. Outcomes for this study should be considered in the context of the given population as this procedure may not be generalizable to other study samples.

\section{Attrition}

One of the primary goals of the present study was to reduce attrition rates for families seeking child mental health services in PCIT. Unfortunately, the use of low cost incentives was found to be ineffective for this community mental health sample. One possibility for the lack of attrition differences between groups receiving and not receiving incentives for treatment adherence may have been that increased oversight by the university-based research team impacted the structure of treatment delivery and enhanced attendance (which would not otherwise be seen in the community mental health agency). This finding is unlikely for a number of reasons. First, considering comparable effectiveness trials in community agency settings, the present study yielded a similar attrition rate of $59.5 \%$ (50 out of 84 families) to other studies evaluating PCIT outcomes (60 - 70\%; Pearl et al., 2012; Stokes et al., in press). Moreover, the research team acquired Riverside agency data on PCIT graduation rates for clients from approximately five years prior to the start of the current project (July, 2011 - March, 2016). The report indicated that $48.6 \%$ of families who initiated PCIT graduated treatment (742 out of 
1527), while the present study yielded a slightly lower graduation rate of $40.5 \%$ (34 out of 84 ). In turn, many families, regardless of group status, dropped out of treatment early (prior to reaching CDI mastery) which is also a common finding in the literature (Liebsack, 2016; McKay et al., 2002). It is believed that conducting the research project at the Riverside agency did not impact client attrition in such a way to explain the lack of differences between incentive groups.

Attrition in PCIT has been linked to a greater number of barriers perceived by families, with many families dropping out in the early stages of treatment (Luk et al., 2001; McCabe, 2002). One theory for early termination stems from Lorion (1974) who stated that many families in poverty are burdened by constant life stressors. The most immediate stressors are prioritized until a more significant stressor arises, the crisis passes, or the crisis remedies into a more manageable degree (Lorion, 1974). In another theory, Maslow's (1943) hierarchy of needs presents human motivation as a five-level model and posits that individuals (or families) are motivated to meet certain needs, with some needs taking priority over others. At the base of the pyramid is a human's physiological need, while the next level up is safety, followed then by love/belonging, esteem, and finally, self-actualization. It is possible then that families who attend PCIT for only a few sessions find enough change in child behavior to cause the need for intervention to be surpassed by a multitude of more pressing crises and concerns (at the safety or physiological level of Maslow's pyramid). Love and belonging within a family may not be an immediately reasonable short-term goal if a parent is struggling with sickness or a child is acting aggressively and refusing to attend treatment. Unfortunately, these competing stressors may continue to prevent families from dedicating adequate resources to attend parent training such as PCIT unless they can either be addressed or additional motivators can help families overcome them. 


\section{Rate of Homework Completion}

Research in PCIT has found that rates of homework completion predict changes in positive parenting skills, parental stress levels, parental satisfaction with treatment, and child behavior problems (Danko et al., 2016; Ros, Hernandez, Graziano, \& Bagner, 2016). Higher rates of homework completion in PCIT also predict the number of sessions to parental skill mastery and graduation from treatment (Stokes et al., 2016). Studies exploring homework completion rates in PCIT have shown large variations between families ranging from $0 \%$ to $86 \%$ (Berkovits et al., 2010; Danko et al., 2016; Lyon \& Budd, 2010), with families dropping out of treatment having lower completion rates ( 0 - 1 days, Danko et al., 2016; 47.4\%, Lyon \& Budd) than families who completed treatment (2 - 6 days, Danko et al., 2016; 62.7\%, Lyon \& Budd, 2010). While incentives were implemented in the current study to increase homework completion rates and improve both caregiver and child outcomes, families only completed paper homework for $1-2$ days per week on average, while audio was completed less frequently at approximately 1 day per week across both groups. Outcomes for reported days of paper homework completion for the present sample add to the continued variability in rates of homework completion across PCIT studies (Berkovits et al., 2010; Danko et al., 2016; Lyon \& Budd, 2010; Ros et al., 2016). On the other hand, audio recordings have not been studied previously in PCIT research. For the present study, audio recordings were added as reliability checks for families claiming to have completed homework. While it was easier for clinicians to determine if clients had actually completed their homework by reviewing the audio recordings, audio recordings posed another barrier to treatment adherence (e.g., having a recording device available, remembering to turn on the recording device, having adequate space or battery life on the recording device). Anecdotal reports from the Riverside staff indicated families completing 
audio homework met mastery criteria at faster rates; however, sample sizes for audio completion were too low to conduct meaningful analyses. In addition, the added requirement of completing audio recordings may not be worthwhile as homework completion rates in the present study were already low. Even with the barriers for implementing audio recordings, further explorations of audio effectiveness in attaining therapeutic skill mastery may be of value.

The low rate of homework completion in PCIT is problematic for a number of reasons. First, families are not adhering to the recommended dose of PCIT, and therefore outcomes are not representative of the treatment's true power. Families who terminate from treatment with the belief that PCIT is ineffective are not technically receiving PCIT as it was intended. Second, families who display higher rates of homework completion obtain improvements on parental and child outcomes more quickly than families who do not complete homework (Stokes et al., 2016). It is possible that families may see greater improvements through treatment attendance and be less likely to terminate if homework was completed at a higher rate. While increased homework practice has been a topic of conversation in psychological treatment for a number of years (see Kazantzis, Deane, \& Ronan, 2000, for a review), still no definitive answer to improve completion rates has yet been determined (Malouff \& Schutte, 2004).

It is important to consider the reasons behind consistently low rates of homework adherence and what this means for PCIT effectiveness in community settings. Providing incentives was proposed as a possible solution to the challenges of low family engagement and poor adherence to homework - assuming these problems stemmed from a lack of motivation. It is quite possible that baseline motivation for families seeking parent training is high, but that barriers complicate the families' ability to attend treatment over time. The incentives delivered in this study did not show any evidence of combatting problems with adherence and engagement in 
PCIT. As such, this study suggests that researchers and clinicians should partner together in future investigations to better understand the function of poor adherence with PCIT in hopes of identifying novel ways to motivate low-resource families to attend sessions regularly, complete homework assignments, and work through the entire course of treatment.

\section{Cost Analysis}

A cost analysis in the present project was run to justify the use of low cost incentives if the prizes that were given had generated greater attendance and general adherence to treatment. Findings from this calculation could have been used to justify the use of incentives as a public policy initiative to reduce lost revenue for mental health agencies, increase long-term outcomes for families, and also reduce future costs for both private and government-based insurance (as reduced behavior problems may lead to less involvement with mental health services over time; see Barrett et al., 2008, for a review; Honberg et al., 2011; Hood \& Eyberg, 2003). Hypothetically, if families were able to adhere to timely attendance and homework completion for every session and were able to attain skill mastery criteria (CDI, PDI) to graduate treatment, families may have cost the Riverside agency anywhere from $\$ 175.50$ to $\$ 279.50$ in prizes (\$6.50/prize across 12 to 20 sessions; Lanier et al., 2011; Zisser \& Eyberg, 2010). These families would, in turn, make the Riverside agency between $\$ 2,905.20$ and $\$ 4,842.00$ (see Results section for cost gained calculation) for coming to sessions, therefore easily justifying the cost of incentives. The low cost of incentives for the present study was used to increase generalizability and dissemination capabilities across agencies in the United States. Although it is possible that more expensive prizes or the use of large monetary incentives may have greater impacts on sustaining families in treatment, limited access to resources and restrictive policies regulating agency spending make this solution both improbable and cost ineffective in community-based 
mental health settings (Dumas et al., 2010; Gross \& Fogg, 2004). Given the null findings from the present study paired with the logistic infeasibility for most agencies, the use of expensive incentives is an unlikely solution to remedy the continued attrition concern.

\section{Barriers to Treatment/Predictors of Family Success}

Researchers have explored a number of factors that contribute to early termination for families in mental health services including variables related to the treatment, therapists, children, and families/parents as well as participation barriers (de Haan et al. 2013). Unfortunately, many of the variables that are explored related to early treatment dropout are stagnant (e.g., socioeconomic status, ethnicity, child gender, age of mother; de Haan et al., 2013; Fernandez \& Eyberg, 2009). While identifying these factors can cue researchers or clinicians in to which families may be more likely to discontinue treatment, they do not allow mental health professionals to actively address these barriers in a meaningful or impactful way. Exploratory analyses were conducted in the present study incorporating some of the most common factors shown to predict attrition. While some of these exploratory variables were related to stagnant child or family characteristics, this discussion is used to understand why these factors may limit treatment attendance. However, creating innovative solutions to overcome barriers that can be altered is a greater focus of this section.

Exploratory analyses in this study examined the impact of caregiver characteristics (e.g., age, income, ethnicity, mental health history), child characteristics (e.g., severity of internalizing and externalizing behaviors), and treatment components and participation barriers that impacted family involvement in PCIT. Analyses found that families of children with more internalizing problems were more likely to terminate from treatment. Although externalizing behaviors are more commonly explored in child populations, greater child internalizing problems have been 
found to significantly predict dropout in outpatient mental health care settings (for a review, see de Haan et al., 2013). However, outcomes related to child internalizing symptomatology are mixed, as some studies have reported higher rates of treatment attendance over time for those with greater internalizing symptoms (Baruch, Vrouva, \& Fearon, 2009; Johnson, Mellor, \& Brann, 2008). One explanation from the literature points to maternal depression (or internal symptomatology) predicting over-reporting of child behavioral concerns (both internalizing and externalizing symptoms) as well as greater termination from treatment (Abrahamse, Niec, Junger, Boer, \& Lindauer, 2016; Fernandez \& Eyberg, 2005; Gartstein, Bridgett, Dishion, \& Kaufman, 2009). It is possible that caregivers in the current sample with depression $(17.9 \% ; n=$ 15) or with any psychopathology $(31.0 \% ; n=26)$ were over-reporting the level of distress the child was experiencing when completing the parent-report measures and subsequently terminated from treatment.

A second explanation for these variations in the literature may be due to the age of youth experiencing internalizing symptoms. Internalizing and externalizing disorders often co-occur and may lead to greater problem behaviors in young children in general (Caron \& Rutter, 1991; Keiley, Lofthouse, Bates, Dodge, \& Pettit, 2003). Moreover, younger children who experience more behavior problems have been found to terminate from treatment earlier than children with less problem behaviors (Abrahamse et al., 2016). It is possible that younger children express their internalizing symptoms in different ways than older children (Tandon, Cardeli, \& Luby, 2009), such that they act out more aggressively and make it more difficult for parents to get them to treatment sessions than older children. Third, the present study found caregivers who dropped out of treatment before reaching CDI mastery stated that their child developed new or different problems since starting treatment. Internalizing symptoms can often present as somatic issues 
with young children (American Psychiatric Association, 2013; Tandon et al., 2009), and it is possible that these issues arose during the initial sessions of PCIT that limited the families' capability to return over time.

Fourth, culture may have greatly contributed to internalizing symptoms impacting treatment attendance. Specifically, other studies have suggested additional factors such as cultural influences, acculturation, and familism play a more significant role in why MexicanAmerican families with high levels of internalizing symptoms drop out of treatment early (Mauricio, Tein, Gonzáles, Millsap, \& Dumka, 2016). Mauricio and colleagues (2016) found that Hispanic families with high levels of internalizing symptomatology that dropped out of treatment were less acculturated and had weaker familism values compared to families with high internalizing but who attended treatment. Moreover, the PCIT adaptation for Hispanic families (Guiando a Niños Activos) has found significantly greater improvements in child internalizing symptomatology than standard PCIT delivery (McCabe, Yeh, Lau, \& Argote, 2012). This outcome may point to a greater need in Hispanic families to address internalizing symptoms that is not addressed in standard PCIT protocol. In the present study, it is possible that cultural influences (i.e., acculturation, familism) may have played a mediating role in impacting treatment termination for children with high internalization symptoms.

Lastly, due to ceiling effects, high rates of externalizing symptomology across children in the present study may have accounted for a lack of termination outcomes related to externalizing symptoms. Since most families were referred to treatment due to the child's clinically significant levels of externalizing behavior problems, differences between children may not have been large enough to detect any effects related to termination status. These individual factors, or the combined influence of these factors, may have contributed to families of children with high 
internalizing symptoms terminating from treatment early. Analyses found that families of mixed ethnicity were also more likely to drop out of treatment compared to Hispanic families. Further information on the specific ethnicities may help explain this finding, but this information was not collected. In addition, this finding should be interpreted with extreme caution due to the small sample size of the "mixed ethnicity" families. Specifically, only six total families out of 84 $(7.1 \%)$ categorized themselves as "mixed," therefore limiting the outcome's implications. Further exploratory analyses were conducted related to participants' feelings toward therapy and their perceived barriers for coming to treatment. Generally, at CDI Coach 3 families reported concerns with children having new or different behavior problems since starting treatment, low rates of child compliance, and high rates of family stress being significant problems in their lives. While individual questions from the Barriers to Treatment Participation Scale have not been looked at across previous evaluations of this measure, these outcomes did reflect general trends from previous attrition literature (Attride-Stirling, Davis, Farrell, Groark, \& Day, 2004; Barrett et al., 2008; Kazdin, Holland, \& Crowley, 1997; McCabe, 2002). Specifically, treatment terminators have been shown to have multiple personal or child difficulties, high levels of stress, and waited to initiate treatment until child problems became unmanageable (Attride-Stirling et al., 2004; Barrett et al., 2008; Kazdin, Holland, \& Crowley, 1997; McCabe, 2002). Interestingly, families in the present study also reported on one of the BTPS items that child symptoms improved over only a few treatment sessions, therefore making PCIT no longer necessary. While adherence to full courses of PCIT have been shown to be highly effective, it is possible that a portion of families terminate treatment due to viewing improvements in their child and overcoming the present behavioral crisis (Lieneman, Olson, Quetsch, Theodorou, \& McNeil, in preparation; Lorion, 1974). 
Preliminary analyses also pointed to families who terminated treatment early having difficulties with child session refusal, new child behavioral problems, and partner disagreement with coming to treatment. While these concerns reflect findings from families regardless of attrition status (as discussed above), each of these items may have uniquely contributed to each family's decision to discontinue treatment. First, child session refusal has been previously shown to be a challenge in attending family therapy courses in child mental health services (McKay et al., 2002). Since the sampled participants were families of children with externalizing behavior problems, it can be assumed that many children had difficulties with defiance or aggression. If children do not see a therapy session as fun or rewarding, they may actively oppose their parents' pleas to travel and engage in a long session therefore making it difficult for parents to overcome this barrier to attend treatment regularly.

Second, new or different child behavior problems were seen as significantly impacting treatment attendance. The author could not find relevant literature emulating this finding. However, research has indicated that parental perceptions of child mental health problems have been strongly correlated with attrition over time in that either the parent believes the child's behaviors are extreme, unmanageable, or will not improve with treatment (McCabe et al., 2005; Owens et al., 2002). In addition, mental health problems are often viewed negatively (as weaknesses) in Hispanic culture as they may not be well understood (Alvidrez, 1999; González, 1997). It is also possible that children developed new concerns once they began treatment, and the externalizing behaviors were no longer the most significant concern or family priority. In this case, treatment for defiance may not have seemed like an effective solution for treatment.

Third, the push-back from other family members who may not agree with PCIT should be emphasized. McCabe (2002) addresses the importance of including immediate and extended 
family members for Hispanic clients seeking mental health services in order to combat negative stereotypes, create a support network, and increase the likelihood that the family remains in treatment. For those families within the current study who terminated later in treatment, issues with parental health were found to be a significant concern. Particular cases such as the mother having baby or undergoing major surgery may explain some of these findings. Mothers in this study were approximately 35 years old and may have been expanding their families. Working with parents with young children who want to improve their child's behavior before introducing a new child into the family may be a common concern that clinicians should consider when delivering PCIT. Additional barriers may also play a role in families' abilities to attend treatment. While issues with transportation, busy schedules, and numerous stressors have been previously reported as significant barriers to attending PCIT sessions (Fernandez \& Eyberg, 2009), these were not rated as highly limiting by the present sample. The families in the current study may not have experienced issues with barriers such as those found previously (Fernandez \& Eyberg, 2009), may not have viewed them as significantly impacting their ability to come to treatment, or may have underreported the impact these factors had on contributing to treatment attendance.

Interestingly though, when comparing total barrier scores to a standardized sample of families of children in outpatient treatment, the current sample of families (across ethnicities) reported significantly fewer barriers (Kazdin, Holland, Crowley, \& Breton, 1997) even though they were comparable across a number of other factors including proportion of dropouts (Present study: 59.5\%: Kazdin study: 61.5\%) and average rate of no-shows per week (Present study: .13; Kazdin study: .15). Kazdin and colleagues reported more canceled sessions per week than the present sample (.46 and .23 , respectively), however. This finding shows that although the current 
sample reported fewer barriers, they still terminated treatment at the same rate as families reporting higher levels of barriers in similar outpatient settings. Importantly, the number of barriers families reported in both samples was high. One possibility for this finding is that barriers to treatment participation play a significant role in early treatment dropout to an extent, but additional barriers past a given level no longer have a compounded impact. Moreover, these high rates of barriers commonly seen in outpatient child mental health services (Kazdin, Holland, Crowley, \& Breton, 1997) may not be overcome with the use of low cost prizes.

Recognizing the complex barriers for families to attend treatment is the first step in addressing treatment attrition, especially given the sample's Hispanic culture, low socioeconomic status, and potentially limited access to resources. Agencies should consider how to work with more than just the primary caregiver to prevent resistance from the caregiver's support system, engage children in the therapy (rather than just working with the parent) to reduce child refusal to come to treatment, constantly communicate with families to check in on child behaviors to help reconceptualize the goals of treatment over time based on the child's needs, and potentially condense treatment to provide key parent training skills within a limited window for pregnant caregivers. Even with the information gained in this study, researchers should continue to explore variables that impact families' abilities to come to treatment. More importantly, alternatives to standard outpatient treatment delivery should be generated to combat the severe issue of barriers preventing families from attending and completing PCIT.

\section{Limitations}

As this study is the first to implement incentives in PCIT, the research involves some exploration and piloting of a large number of initial research questions. Unfortunately, to address the study's aims using stronger analyses such as MANOVAs, mediations, or moderations, a 
larger number of participants would have been required to have adequate power and effect sizes. Due to the unique nature of this study and the collaboration with a community mental health agency, the final sample for this study was large relative to other studies conducted with PCIT but not adequate to conduct more powerful analyses. The researcher is aware that all findings must be interpreted with caution due to the number of questions utilized to assess outcomes and feasibility. While multiple analyses are often subject to Type I error, the present study had issues with low power due to high rates of attrition. This prevented the conducted analyses from having the appropriate sample size to determine outcomes. In this case, Type II error may have played a more significant role (with power as low as .35 in some cases).

Another limitation to the present study was the sample size difference between incentive and nonincentive groups. The current study's procedure included the restricted random assignment of families through a computer-generated assignment program. Families were given a number randomly generated once they contacted the community mental health agency. Odd numbers from the computer were assigned to the PCIT plus incentives group, while even numbers were indicative of the PCIT as usual group. A supervisor double checked the group each family was assigned based on the community identification number before treatment started (i.e., during the first assessment). A new participant number was then assigned to the family for the purposes of the study. The community identification number was not included in data collection. Unfortunately, even though the lead researcher implemented thorough evaluations of the enrollment protocol and randomization checks within the agency over the course of the study, the groups still varied in size. Also, since the researchers were not directly involved in the assignment of families to groups and de-identified data was sent to remove any identifiers from study participants, agency self-report of accurate assignment may have been violated without the 
researcher's knowledge. In addition, the socioeconomic status (although not significant) was largely varied between the two groups with the nonincentive group having a higher income $(M=$ $22,514 ; S D=21,992)$ than the incentive group $(M=33,562 ; S D=25,720)$. Given the low income level for the entire sample, a $\$ 10,000$ difference in income is substantial when comparing between the two groups. Outcomes in the present study are limited as the randomization of the sample may have been compromised. In turn, the difference in sizes between groups may have altered the accurate representation of the sampled population, yielded different outcomes on measured variables, and changed the mean comparisons on present analyses. Lastly, although the internal consistency of the items for the total Therapy Attitude Inventory and Barriers to Treatment Participation Scale were adequate and did not differ between the Hispanic and nonHispanic participants, a measurement limitation occurred in this study because the Barriers to Treatment Participation subscales demonstrated low Cronbach's alpha scores suggesting poor scale reliability.

Finally, due to the nature of conducting research in a community-based mental health setting, concerns related to fidelity must be considered. Although the researcher was not present during each clinical session conducted, she did have an on-site supervisor to manage study adherence to both the study protocol and PCIT protocol. In addition, she led weekly phone consultation meetings to answer study questions and address concerns raised while reviewing any collected data. Lastly, each Session Record Form included checks for fidelity to the model (e.g., providing incentives) that was monitored by both the on-site supervisor and checked by the lead researcher. Although these checks provide a safeguard against contamination or integrity issues in the treatment delivery, the possibility remains that therapists may have veered from study procedures when working in the community with individual families. 


\section{Clinical Implications and Future Research}

Overall, positive outcomes were found for changes in internalizing and externalizing child behavior problems as well as parental stress across the sampled families. While consistent with the larger literature on PCIT (Chase \& Eyberg, 2008), these findings are unique as this is one of the few PCIT studies conducted with a largely Hispanic population (McCabe, 2002; McCabe et al., 2005), and one of the only RCTs with this population as well (McCabe \& Yeh, 2009; McCabe et al., 2012). Since much of PCIT was delivered in Spanish, this study lends support for the effectiveness of PCIT delivered in this way. As a whole, this study indicates that PCIT shows great promise for Hispanic families.

Providing larger incentives may be a possible next step to increase family adherence to PCIT treatment components, yet the literal costs may outweigh the benefits. The current project was conducted with the goal of making a feasible, low cost option for therapists and administrators looking to reduce attrition within their agencies. While large research-funded projects may be able to address the question "how much is enough," this may not provide longterm solutions for realistic sustainability efforts. Alternative approaches such as monetary incentives, gift cards, tokens for a within-clinic store, or a family's ability to select their prizes might yield different results; however, no benefits were found based on the present study's evaluations. Future studies could explore the alternative options for incentive-based prizes in addition to larger prizes.

As incentives were not successful in this study, future research should explore other means for addressing the problematic rates of attrition in PCIT and child mental health services. Some cutting-edge solutions have been tested to tackle barriers to treatment participation and issues with treatment maintenance. Specifically, Chaffin and colleagues (2009) found that using 
motivational interviewing techniques prior to starting PCIT for families referred to child welfare for neglect could improve retention rates. However, this finding was only significant for families who started with low to moderate motivation. When implemented with highly motivated parents, motivational interviewing yielded detrimental outcomes. Used selectively, motivational interviewing may greatly impact family retention in PCIT for families with low motivation levels. More recently, PCIT researchers in Australia incorporated motivational interviewing and enhancement techniques prior to starting standard treatment protocol (Webb, Thomas, McGregor, Avdagic, \& Zimmer-Gembeck, 2017). Although Webb and colleagues witnessed a change in parental motivation for those families receiving the motivational enhancement sessions, no differences were found between standard PCIT and motivationally-enhanced PCIT when comparing retention rates over time. Given the paucity of studies including motivational components, continued advancements in the arena of motivational enhancements should be pursued.

Another innovation to the delivery of PCIT is the implementation of treatment in client homes. While the literature has stated for a long time that transportation to outpatient clinics for treatment is a barrier to session attendance (Kolko, Parrish, \& Wilson, 1985), researchers are just recently starting to move in the direction of home-based services (see Masse, Quetsch, \& McNeil, in press, for an overview). While some projects have found insufficient results to justify the added agency burden of providing in-home therapy (Lanier et al., 2011; Timmer, Zebell, Culver, \& Urquiza, 2010), other studies are yielding successful outcomes. For example, families in Fowles and colleagues' study (2017) who received home-based services were two times more likely to complete treatment than families attending PCIT in clinic-based settings. In addition, families have stated high rates of satisfaction for this delivery method (Galanter et al., 2012). 
Continued research should explore factors contributing to successful implementation of PCIT within the home.

On top of home-based treatment, innovations in technology are also advancing treatment delivery options in child mental health research. Comer and colleagues recently evaluated homebased Internet-delivered Parent-Child Interaction Therapy (I-PCIT) in a randomized trial (Comer et al., 2015; Fleming, Kimonis, Datyner, \& Comer, 2017). The researchers studied videoteleconferencing to deliver PCIT with a clinic-based live therapist to a client in the home. Posttreatment evaluations indicated significantly more families in I-PCIT had excellent ratings of treatment response and fewer parent-reported barriers to treatment participation than families receiving clinic-based PCIT (Comer et al., 2015). In a case study done with I-PCIT, the child had significant improvements in disruptive behavior that maintained at follow-up (Fleming et al., 2017). While preliminary, utilizing technology in treatment delivery points to a promising future that addresses concerns often found in parent training delivery.

An additional delivery option for PCIT is the implementation of an intensive PCIT course. Proposed by Graziano and colleagues (2015), intensive PCIT consisted of 90 minute appointments taking place over the course of two weeks. The 10 sessions were broken into 1 CDI Teach, followed by 4 CDI coaches, 1 PDI Teach, and finally 4 PDI coaches. In this version of PCIT, CDI and PDI mastery were not required for the completion of treatment. Results indicated high levels of attendance, satisfaction, positive change in child externalizing behaviors, improvements in parenting skills, and reductions in parental stress (Graziano et al., 2015). In fact, effect sizes in the intensive PCIT were larger than those reported by standard PCIT for child compliance, parent-report of child externalizing behaviors, maintenance of family gains, and parental skill acquisition. By creating a shortened version of the highly effective treatment, 
therapists may be able to overcome reported barriers such as parental sickness (e.g., parental pregnancy), child session refusal that could occur over longer treatment periods, or the possibility of new child behavior problems arising in this time. Implementing a condensed version of PCIT may be a more viable option for families as they struggle to continue to come to treatment over time.

Lastly, dissemination and implementation science has recognized the importance of evaluating how agency-level and therapist-level barriers may influence family perceptions of treatment and engagement over time. For example, time on waiting lists, poor therapeutic alliance, and poor therapeutic relationship have all been found as factors significantly affecting treatment attrition (de Haan et al., 2013; Robbins et al., 2006; Russell, Lang, \& Brett, 1987). The delivery of short, intensive treatments that address family barriers to treatment (e.g., transportation, family member resistance) in early clinic sessions have shown valuable changes in early treatment engagement as well (Ingoldsby, 2010). While incentives in the present study were not found to be effective on measured variables, understanding the complexities of family barriers and continuing to approach solutions to attrition in mental health care may lead to better delivery methods and outcomes for families in the future. 


\section{References}

Abidin, R. R. (1990). Parenting Stress Index / Short Form. Lutz, FL: Psychological Assessment Resources, Inc.

Abidin, R. R. (1995). Parenting Stress Index (PSI) manual (3rd ed.). Charlottesville, VA: Pediatric Psychology Press.

Abrahamse, M. E., Junger, M., Chavannes, E. L., Coelman, F. J., Boer, F., \& Lindauer, R. J. (2012). Parent-Child Interaction Therapy for preschool children with disruptive behaviour problems in the Netherlands. Child Adolescent Psychiatry Mental Health, 6 (1), 24.

Abrahamse, M. E., Niec, L N., Junger, M., Boer, F., \& Lindauer, R. J. L. (2016). Risk factors for attrition from an evidence-based parenting program: Findings from the Netherlands. Children and Youth Services Review, 64, 42-50.

Achenbach, T. M., Dumenci, L., \& Rescorla, L. A. (2003). DSM oriented and empirically based approaches to constructing scales from the same item pools. Journal of Clinical Child \& Adolescent Psychology, 32, 328-340.

Achenbach, T. M., \& Rescorla, L. A. (2001). Manual for ASEBA school-aged forms \& profiles. Burlington, VT: University of Vermont, Research Center for Children, Youth, \& Families.

Agazzi, H., Tan, S. Y., Ogg, J., Armstrong, K., \& Kirby, R. S. (2017). Does Parent-Child Interaction Therapy reduce maternal stress, anxiety, and depression among mothers of children with autism spectrum disorder? Child \& Family Behavior Therapy, 39(4), 283303. 
Alvidrez, J. (1999). Ethnic variations in mental health attitudes and service use among lowincome African American, Latina, and European American young women. Community Mental Health Journal, 35, 515-530.

American Psychiatric Association (2013). Diagnostic and statistical manual of mental disorders. (5th ed., text revision). Washington, DC: American Psychiatric Association.

Armbruster, P., \& Kazdin, A. E. (1994). Attrition in child therapy. In T. H. Ollendick, \& T. J. Prinz (Eds.), Advances in clinical child psychology, Vol. 16. (pp. 81-108). New York: Plenum.

Attride-Stirling, J., Davis, H., Farrell, L., Groark, C., \& Day, C. (2004). Factors influencing parental engagement in a community child and adolescent mental health service: A qualitative comparison of completers and non-completers. Clinical Child Psychology and Psychiatry, 9(3), 349-361.

Baker, C. N., Arnold, D. H., \& Meagher, S. (2011). Enrollment and attendance in a parent training prevention program for conduct problems. Prevention Science, 12(2), 126-138.

Baker-Ericzén, M. J., Hurlburt, M. S., Brookman-Frazee, L., Jenkins, M. M., \& Hough, R. L. (2010). Comparing child, parent, and family characteristics in usual care and empirically supported treatment research samples for children with disruptive behavior disorders. Journal of Emotional and Behavioral Disorders, 18, 82-99.

Baker-Ericzén, M., Jenkins, M., \& Haine-Schlagel, R. (2013). Therapist, parent, and youth perspectives of treatment barriers to family-focused community outpatient mental health services. Journal of Child \& Family Studies, 22(6), 854- 868. 
Barnett, M. L., Niec, L. N., Peer, S. O., Jent, J. F., Weinstein, A., Gisbert, P., \& Simpson, G. (2017). Successful therapist-parent coaching: How in vivo feedback relates to parent engagement in Parent-Child Interaction Therapy. Journal of Clinical Child and Adolescent Psychology, 46(6), 895-902.

Barrett, M. S., Chua, W., Crits-Christoph, P., Gibbons, M. B., Casiano, D., \& Thompson, D. (2008). Early withdrawal from mental health treatment: Implications for psychotherapy practice. Psychotherapy Theory, Research, Practice, Training, 45(2), 247-267.

Baruch, G., Vrouva, I., \& Fearon, P. (2009). A follow-up study of characteristics of young people that dropout and continue psychotherapy: Service implications for a clinic in the community. Child and Adolescent Mental Health, 14(2), 69-75.

Bean, A. G., \& Talaga, J. (1992). Appointment breaking: Cases and solutions. Journal of Health Care Marketing, 12, 12-14.

Berkovits, M. D., O’Brien, K. A., Carter, C. G., \& Eyberg, S. M. (2010). Early identification and intervention for behavior problems in primary care: A comparison of two abbreviated versions of Parent-Child Interaction Therapy. Behavior Therapy, 41, 375-387.

Bjorseth, A., Wormdal, A. K., \& Chen, Y. (2010). PCIT around the world. In T. L. HembreeKigin \& C. B. McNeil (Eds.). Parent-child interaction therapy: Expanded clinical edition (pp. 421-427). New York: Springer.

Blair, C., \& Diamond, A. (2008). Biological processes in prevention and intervention: The promotion of self-regulation as a means of preventing school failure. Development \& Psychopathology, 20(3), 899-911. 
Boggs, S. R., Eyberg, S. M., Edwards, D. L., Rayfield, A., Jacobs, J., Bagner, D., \& Hood, K. K. (2004). Outcomes of Parent-Child Interaction Therapy: A comparison of dropouts and treatment completers one to three years after treatment. Child \& Family Behavior Therapy, 26, 1-22.

Boggs, S. R., Eyberg, S. M., \& Reynolds, L. (1990). Concurrent validity of the Eyberg Child Behavior Inventory. Journal of Clinical Child Psychology, 19, 75-78.

Borrego, J., Anhalt, K., Terao, S. Y., Vargas, E. C., \& Urquiza, A. J. (2006). Parent-Child Interaction Therapy with a Spanish-speaking family. Cognitive and Behavioral Practice, $13,121-133$.

Brestan, E. V., Eyberg, S. M., Boggs, S., \& Algina, J. (1997). Parent-Child Interaction Therapy: Parent perceptions of untreated siblings. Child \& Family Behavior Therapy, 19, 13-28.

Brestan, E. V., Jacobs, J. R., Rayfield, A. D., \& Eyberg, S. M. (1999). A consumer satisfaction measure for parent-child treatments and its relation to measures of child behavior change. Behavior Therapy, 30(1), 17-30.

Bride, B. E., \& Humble, M. N. (2008). Increasing retention of African-American women on welfare in outpatient substance user treatment using low-magnitude incentives. Substance Use \& Misuse, 43(8-9), 1016-1026.

Butler, A. M., \& Eyberg, S. M. (2006). Parent-Child Interaction Therapy and ethnic minority children. Vulnerable Children and Youth Studies, 1(3), 246-255.

Capage, L. C., Bennett, G. M., \& McNeil, C. B. (2001). A comparison between African American and Caucasian children referred for treatment of disruptive behavior disorders. Child \& Family Behavior Therapy, 23(1), 1-14. 
Capaldi, D. M., \& Clark, S. (1998). Prospective family predictors of aggression toward female partners for at-risk young men. Developmental Psychology, 34 (6), 1175-1188.

Cardona, J. R. P., Domenech-Rodriguez, M., Forgatch, M., Sullivan, C., Bybee, D., Holtrop, K., Escobar-Chew, A. R., Tams, L., Dates, B., \& Bernal, G. (2012). Culturally adapting an evidence-based parenting intervention for Latino immigrants: The need to integrate fidelity and cultural relevance. Family Process, 51, 56-72.

Carey, K. B., \& Carey, M. P. (1990). Enhancing the treatment attendance of mentally ill chemical abusers. Journal of Behavior Therapy \& Experimental Psychiatry, 21, 205-209.

Caron, C., \& Rutter, M. (1991). Comorbidity in child psychopathology: Concepts, issues, and research strategies. Journal of Child Psychology \& Psychiatry, 32(7), 1063-1080.

Chacko, A., Wymbs, B. T., Wymbs, F. A., Pelham, W. E., Swanger-Gagne, M. S., Girio, E., \& O’Connor, B. (2009). Enhancing traditional behavioral parent training for single mothers of children with ADHD. Journal of Clinical Child and Adolescent Psychology, 38, 206218.

Chaffin, M., Valle, L. A., Funderburk, B., Gurwitch, R., Silovsky, J., Bard, D., McCoy, C., \& Kees, M. (2009). A motivational intervention can improve retention in PCIT for lowmotivation child welfare clients. Child Maltreatment, 14(4), 356-368.

Chase, R. M., \& Eyberg, S. M. (2008). Clinical presentation and treatment outcome for children with comorbid externalizing and internalizing symptoms. Anxiety Disorders, 22, 273282.

Cheng, Y. C., \& Yeh, H. T. (2009). From concepts of motivation to its application in instructional design: Reconsidering motivation from an instructional design perspective. British Journal of Education Technology, 40(4), 597-605. 
Chutuape, M. A., Katz, E. C., \& Stitzer, M. L. (2001). Methods for enhancing transition of substance dependent patients from inpatient to outpatient treatment. Drug \& Alcohol Dependence, 61, 137-143.

Cohen, J. A., Mannarino, A. P., \& Knudsen, K. (2005). Treating sexually abused children: 1 year follow-up of a randomized controlled trial. Child Abuse \& Neglect, 29, 135-145.

Colvin, A., Eyberg, S. M., \& Adams, C. (1999). Restandardization of the Eyberg Child Behavior Inventory. Gainesville: University of Florida, Child Study Laboratory. Retrieved from http://pcit.phhp.ufl.edu/Measures.htm

Comer, J. S., Furr, J. M., Miguel, E. M., Carpenter, A. L., Kerns, C. E., DeSerisy, M.,...Chase, R. (2015). Remotely delivering real-time parent training to the home: An initial randomized trial of Internet-delivered Parent-Child Interaction Therapy (I-PCIT). Journal of Consulting and Clinical Psychology, 25(9), 909-917.

Danko, C. M., Brown, T., Van Schoick, L., \& Budd, K. S. (2016). Predictors and correlates of homework completion and treatment outcomes in Parent-Child Interaction Therapy. Child Youth Care Forum, 45, 467-485.

de Haan, A. M., Boon, A. E., de Jong, J. T., Hoeve, M., \& Vermeiren, R. R. J. M. (2013). A meta-analytic review on treatment dropout in child and adolescent outpatient mental health care. Clinical Psychology Review, 33, 698-711.

Deci, E. L., Koestner, R., \& Ryan, R. M. (2001). Extrinsic rewards and intrinsic motivation in education: Reconsidered once again. Review of Educational Research, 71(1), 1-27.

Deci, E. L., \& Ryan, R. M. (1980). The empirical exploration of intrinsic motivational processes. In L. Berkowitz (Ed.), Advances in experimental social psychology (Vol. 13, pp. 39-80). New York: Academic Press. 
Donovan, D. M. (2003). Relapse prevention in substance abuse treatment. In J. L. Sorensen, R. A. Rawson, J. Guydish, \& J. E. Zweben (Eds.), Drug abuse treatment through collaboration: Practice and research partnerships that work (pp. 121-137). Washington, DC: American Psychological Association.

Drummond, M. F., O’Brien, B. J., Sculpher, M. J., Stoddart, G. L., \& Torrance, G. W. (2005). Methods for the economic evaluation of health care programmes (3rd ed.). New York: Oxford University Press.

Dumas, J. E., Begle, A. M., French, B. F., \& Pearl, A. M. (2010). Effects of monetary incentives on engagement in the PACE parenting program. Journal of Clinical Child \& Adolescent Psychology, 39, 302-313.

Eisenberger, R., \& Cameron, J. (1996). Detrimental effects of reward: Reality or myth? American Psychologist, 51, 1153-1166.

Eisenstadt, T. H., Eyberg, S., McNeil, C. B., Newcomb, K., \& Funderburk, B. (1993). ParentChild Interaction Therapy with behavior problem children: Relative effectiveness of two stages and overall treatment outcome. Journal of Clinical Child Psychology, 22(1), 4251.

Eisenstadt, T. H., McElreath, L. S., Eyberg, S. M., \& McNeil, C. B. (1994). Interparent agreement on the Eyberg Child Behavior Inventory. Child \& Family Behavior Therapy, $16,21-28$.

Eyberg, S. M. (1993). Consumer satisfaction measures for assessing parent training programs. In L. VandeCreek, S. Knapp, \& T. L. Jackson (Eds.), Innovations in clinical practice: A source book (Vol. 12). Sarasota, FL: Professional Resource Press. 
Eyberg, S. M., Edwards, D., Boggs, S., \& Foote, R. (1998). Maintaining the treatment effects of parent training: The role of booster sessions and other maintenance strategies. Clinical Psychology: Science \& Practice, 5, 544-554.

Eyberg, S. M., \& Funderburk. B. (2011). Parent-Child Interaction Therapy protocol. PCIT International: Gainesville, FL.

Eyberg, S. M., Funderburk, B., Hembree-Kigin, T., McNeil, C. B., Querido, J., \& Hood, K. (2001). Parent-Child Interaction Therapy with behavior problem children: One and two year maintenance of treatment effects in the family. Child \& Family Behavior Therapy, $23(4), 1-20$.

Eyberg, S. M., \& Johnson, S. M. (1974). Multiple assessment of behavior modification with families: Effects of contingency contracting and order of treating problems. Journal of Consulting \& Clinical Psychology, 42(4), 594-606.

Eyberg, S. M., Nelson, M. M., Duke, M., \& Boggs, S. R. (2005). Manual for the dyadic parentchild interaction coding system (3rd ed.). Retrieved from http://www.PCIT.org

Eyberg, S. M., Nelson, M. M., \& Boggs, S. R. (2008). Evidence-based psychosocial treatments for children and adolescents with disruptive behavior. Journal of Clinical Child \& Adolescent Psychology, 37(1), 215-237.

Eyberg, S. M., Nelson, M. M., Ginn, N. C., Bhuiyan, N., \& Boggs, S. R. (2013). Dyadic ParentChild Interaction Coding System: Comprehensive manual for research and training $\left(4^{\text {th }}\right.$ ed.). Gainesville, FL: PCIT International.

Eyberg, S. M., \& Pincus, D. (1999). Eyberg Child Behavior Inventory and Sutter-Eyberg Behavior Inventory-Revised: Professional manual. Odessa, FL: Psychological Assessment Resources. 
Eyberg, S. M., \& Robinson, E. A. (1983). Conduct problem behavior: Standardization of a behavioral rating scale with adolescents. Journal of Clinical Child Psychology, 12, 347354.

Fernandez, M. A., \& Eyberg, S. M. (2009). Predicting treatment and follow-up attrition in Parent-Child Interaction Therapy. Journal of Abnormal Child Psychology, 37, 431-441.

Flannery-Schroeder, E., Choudhury, M. S., \& Kendall, P. C. (2005). Group and individual cognitive-behavioral treatments for youth with anxiety disorders: 1-year follow-up. Cognitive Therapy and Research, 29, 253-259.

Fleming, G. E., Kimonis, E. R., Datyner, A., \& Comer, J. S. (2017). Adapting internet-delivered Parent-Child Interaction Therapy to treat co-occurring disruptive behavior and callousunemotional traits: A case study. Clinical Case Studies, 16(5), 370-387.

Fowles, T. R., Masse, J. J., McGoron, L., Beveridge, R. M., Williamson, A. A., Smith, M. A., \& Parrish, B. P. (2017). Home-based vs. clinic-based Parent-Child Interaction Therapy: Comparative effectiveness in the context of dissemination and implementation. Journal of Child and Family Studies, (online version), 1-15.

Frye, R. (2011). Financial incentives and student achievement: Evidence from randomized trials. Quarterly Journal of Economics, 126(4), 1755-1798.

Funderburk, B. W., Eyberg, S. M., Rich, B. A., \& Behar, L. (2003). Further psychometric evaluation of the Eyberg and Behar rating scales for parents and teachers of preschoolers. Early Education \& Development, 14, 67-81.

Galanter, R., Self-Brown, S., Valente, J. R., Dorsey, S., Whitaker, D. J., Bertuglia-Haley, M., \& Prieto, M. (2012). Effectiveness of Parent-Child Interaction Therapy delivered to at-risk families in the home setting. Child \& Family Behavior Therapy, 34(3), 177-196. 
Gallagher, N. (2003). Effects of Parent-Child Interaction Therapy on young children with disruptive behavior disorders. Bridges, 1(4), 1-17.

Garfield, S. L. (1994). Research on client variables in psychotherapy. In A. E. Bergin, \& S. L. Garfield (Eds.), Handbook of psychotherapy and behavior change (4th Edition) (pp. 190-228). New York: John Wiley \& Sons.

Gartstein, M. A., Bridgett, D. J., Dishion, T. J., \& Kaufman, N. K. (2009). Depressed mood and maternal report of child behavior problems: Another look at the depression-distortion hypothesis. Journal of Applied Developmental Psychology, 30(2), 149-160.

Gau, S. S. F., Chong, M., Yang, P., Yen, C., Liang, K. Y., \& Cheng, A. T. A. (2007). Psychiatric and psychosocial predictors of substance use disorders among adolescents: Longitudinal study. The British Journal of Psychiatry, 190, 42-48.

Goldfine, M. E., Wagner, S. M., Branstetter, S. A., \& McNeil, C. B. (2008). Parent-Child Interaction Therapy: An examination of cost-effectiveness. Journal of Early \& Intensive Behavior Intervention, 5(1), 119-141.

González, G. M. (1997). The emergence of Chicanos in the twenty-first century: Implications for counseling, research, and policy. Journal of Multicultural Counseling and Development, $25,94-106$.

Graziano, P. A., Bagner, D. M., Slavec, J., Hungerford, G., Kent, K., Babinski, D., Derefinko, K., \& Pasalich, D. (2015). Feasibility of intensive Parent-Child Interaction Therapy (IPCIT): Results from an open trial. Journal of Psychopathology and Behavioral Assessment, 37(1), 38-49.

Gross, D., \& Fogg, L. (2004). A critical analysis of the intent-to-treat principle in prevention research. Journal of Primary Prevention, 25, 475-489. 
Guyll, M., Spoth, R., \& Redmond, C. (2003). The effects of incentives and research requirements on participation rates for a community-based preventive intervention research study. Journal of Primary Prevention, 24, 25-41.

Haine-Schlagel, R., \& Walsh, N. E. (2015). A review of parent participation engagement in child and family mental health treatment. Clinical Child \& Family Psychology Review, $18(2), 133-150$.

Harwood, M. D., \& Eyberg, S. M. (2004). Therapist verbal behavior early in treatment: Relation to successful completion of Parent-Child Interaction Therapy. Journal of Child \& Adolescent Psychology, 33, 601-612.

Harwood, M. D., \& Eyberg, S. M. (2006). Child-directed interaction: Prediction of change in impaired mother-child functioning. Journal of Abnormal Child Psychology, 34(3), 335347.

Hayes, R. A., Efron, L. A., Richman, G. S., Harrison, K. A., \& Aguilera, E. L. (2000). The effects of behavioural contracting and preferred reinforcement on appointment keeping. Behaviour Change, 17(2), 90-96.

Heinrichs, N. (2006). The effects of two different incentives on recruitment rates of families into a prevention program. Journal of Primary Prevention, 27, 345-365.

Heinrichs, N., \& Jensen-Doss, A. (2010). The effects of incentives on families' long-term outcome in a parenting program. Journal of Clinical Child \& Adolescent Psychology, 39, $705-712$.

Heinrichs, N., Kliem, S., \& Hahlweg, K. (2014). Four-year follow-up f a randomized controlled trial of Triple P group for parent and child outcomes. Prevention Science, 15(2), 233-245. 
Hoffman, C., Crnic, K., \& Baker, J. K. (2006). Maternal depression and parenting: Implications for children's emergent emotion regulation and behavioral functioning. Parenting: Science and Practice, 6, 271-295.

Honberg, R., Diehl, S., Kimball, A., Gruttadaro, D., \& Fitzpatrick, M. (2011). State mental health cuts: A national crisis. National Alliance on Mental Illness (NAMI). Report.

Hood, K. K., \& Eyberg, S. M. (2003). Outcomes of Parent-Child Interaction Therapy: Mothers' reports of maintenance three to six years after treatment. Journal of Clinical Child \& Adolescent Psychology, 32(3), 419-429.

Iguchi, M. Y., Belding, M. A., Morrel, A. R., \& Lamb, R. J. (1997). Reinforcing operants other than abstinence in drug abuse treatment: An effective alternative for reducing drug use. Journal of Consulting and Clinical Psychology, 65, 421-428.

Ingoldsby, E. M. (2010). Review of interventions to improve family engagement and retention in parent and child mental health programs. Journal of Child and Family Studies, 19(5), 629-645.

Johnson, E., Mellor, D., \& Brann, P. (2008). Differences in dropout between diagnoses in child and adolescent mental health services. Clinical Child Psychology and Psychiatry, 13(4), $515-530$.

Joshi, P. K., Maisami, M., \& Coyle, J. T. (1986). Prospective study of intake procedures in a child psychiatry clinic. Journal of Clinical Psychiatry, 47, 111-113.

Kaminski, J. W., Valle, L. A., Filene, J. H., \& Boyle, C. L. (2008). A meta-analytic review of components associated with parent training program effectiveness. Journal of Abnormal Child Psychology, 36(4), 567-589. 
Kazantzis, N., Deane, F. P., \& Ronan, K. R. (2000). Homework assignments in cognitive and behavioral therapy: A meta-analysis. Clinical Psychology: Science and Practice, 7(2), 189-202.

Kazantzis, N., Deane, F. P., Ronan, K. R., \& L'Abate, L. (Eds.) (2005). Using homework assignments in cognitive behavioral therapy. New York: Routledge.

Kazantzis, N., Whittington, C., \& Dattilio, F. (2010). Meta-analysis of homework effects in cognitive and behavioral therapy: A replication and extension. Clinical Psychology: Science and Practice, 17, 144-156.

Kazdin, A. E. (1988). Child psychotherapy: Developing and identifying effective treatments. Elmsford, NY: Pergamon Press.

Kazdin, A. E., Holland, L., Crowley, M., \& Breton, S. (1997). Barriers to treatment participation scale: Evaluation and validation in the context of child outpatient treatment. Journal of Child Psychology \& Psychiatry, 38(8), 1051-1062.

Kazdin, A. E., Holland, L., \& Crowley, M. (1997). Family experience of barriers to treatment and premature termination from child therapy. Journal of Consulting \& Clinical Psychology, 65, 453-463.

Kazdin, A. E., \& Mazurick, J. L. (1994). Dropping out of child psychotherapy: Distinguishing early and late dropouts over the course of treatment. Journal of Consulting \& Clinical Psychology, 62, 1069-1074.

Kazdin, A. E., \& Whitley, M. K. (2006). Comorbidity, case complexity, and effects of evidencebased treatment for children referred for disruptive behavior. Journal of Consulting \& Clinical Psychology, 74, 455-467. 
Keiley, M. K., Lofthouse, N., Bates, J. E., Dodge, K. A., \& Pettit, G. S. (2003). Differential risks of co-varying and pure components in mother and teacher reports of externalizing and internalizing behavior across ages 5 to 14. Journal of Abnormal Child Psychology, 31(3), $267-283$.

Kidorf, M., Stitzer, M. L., Brooner, R. K., \& Goldberg, J. (1994). Contingent methadone takehome doses reinforce adjunct therapy attendance of methadone maintenance patients. Drug and Alcohol Dependence, 36, 221-226.

Kim, H., Munson, M. R., \& McKay, M. M. (2012). Engagement in mental health treatment among adolescents and young adults: A systematic review. Child and Adolescent Social Work Journal, 29, 241-266.

Klein, E. P. (2014). Patient health incentives: Ethical challenges and frameworks. International Journal of Behavioral Medicine, 21(6), 995-1004.

Klein, E. B., Stone, W. N., Hicks, M.W., \& Pritchard, I. L. (2003). Understanding dropouts. Journal of Mental Health Counseling, 25, 89-100.

Kling, A., Forster, M., Sundell, K., \& Melin, L. (2010). A randomized controlled effectiveness trial of parent management training with varying degrees of therapist support. Behavior Therapy, 41, 530-542.

Kohn, A. (1993). Punished by rewards. Boston: Houghton Mifflin Company.

Kolko, D., Parrish, J. M., \& Wilson, F. E. (1985). Obstacles to appointment keeping in a child behavior management clinic. Child \& Family Behavior Therapy, 7(1), 9-15.

Lanier, P., Kohl, P. L., Benz, J., Swinger, D., Moussette, P., \& Drake, B. (2011). Parent-Child Interaction Therapy in a community setting: Examining outcomes, attrition, and treatment setting. Research on Social Work Practice, 21(6), 689-698. 
Leung, C., Tsang, S., Heung, K., \& Yiu, I. (2009). Effectiveness of Parent-Child Interaction Therapy (PCIT) among Chinese families. Research on Social Work Practice, 19, 304313.

Liebsack, B. K. (2016). Attrition in Parent-Child Interaction Therapy. Available from Dissertations \& Theses at West Virginia University; ProQuest Dissertations \& Theses Full Text.

Lieneman, C., Olson, R., Quetsch, L. B., Theodorou, L., \& McNeil, C. B. (in preparation). Reconceptualizing attrition in PCIT: Dropouts demonstrate impressive improvements in behavior.

Lippman, D. (2012). State mental health cuts hit low-income patients hard. Retrieved August 19, 2015, from http://www.huffingtonpost.com/2012/09/19/state-mental-healthcuts_n_1897769.html

Loeber, R., Burke. J. D., Lahey, B. B., Winters, A., \& Zera, M. (2000). Oppositional defiant and conduct disorder: A review of the past 10 years, part I. Journal of the American Academy of Child \& Adolescent Psychiatry, 39, 1468-1484.

Lorion, R. P. (1974). Patient and therapist variables in the treatment of low-income patients. Psychological Bulletin, 81, 344-354.

Loyd, B. H., \& Abidin, R. B. (1985). Revision of the Parenting Stress Index. Journal of Pediatric Psychology, 10, 169-177.

Luk, E. S. L., Staiger, P. K., Mathai, J., Wong, L., Birleson, P., \& Adler, R. (2001). Children with persistent conduct problems who dropout of treatment. European Child \& Adolescent Psychiatry, 10, 28-36. 
Lyon, A. R., \& Budd, K. S. (2010). A community mental health implementation of Parent-Child Interaction Therapy (PCIT). Journal of Child \& Family Studies, 19(5), 654-668.

Malouff, J. M., \& Schutte, N. S. (2004). Strategies for increasing client completion of treatment assignments. The Behavior Therapist, 27(6), 118-121.

Martínez-Taboas, A., Cordero-Soto, M. M., Dávila, T., Oben, A., Pujals, K., Medina, M., Saez Colón, J., \& Romaguera, A. (2014). Gifts in psychotherapy: Attitudes and experiences of Puerto Ricans psychotherapists. Revista Puertoreiqueña de Psicología, 25(2), 328-339.

Maslow, A. H. (1943). A theory of human motivation. Psychological Review, 50, 370-396.

Masse, J., Quetsch, L. B., \& McNeil, C. B. (in press). Home-based implementation of ParentChild Interaction Therapy. In L. Niec (Ed.), Handbook of Parent-Child Interaction Therapy: Innovations and applications for research and practice. New York, NY: Springer.

Matos, M., Bauermeister, J. J., \& Bernal, G. (2009). Parent-Child Interaction Therapy for Puerto Rican preschool children with ADHD and behavior problems: A pilot efficacy study. Family Process, 48(2), 232-252.

Mauricio, A. M., Tein, J., Gonzáles, N. A., Millsap, R. E., \& Dumka, L. E. (2016). Attendance patterns and links to non-response on child report of internalizing among MexicanAmericans randomized to a universal preventive intervention. Prevention Science, 1-11.

Mausbach, B. T., Moore, R., Roesch, S., Cardenas, V., \& Patterson, T. L. (2010). The relationship between homework compliance and therapy outcomes: An updated metaanalysis. Cognitive Therapy and Research, 34, 429-438.

McCabe, K. M. (2002). Factors that predict premature termination among Mexican-American children in outpatient psychotherapy. Journal of Child and Family Studies, 11, 347-359. 
McCabe, K., \& Yeh, M. (2009). Parent-Child Interaction Therapy for Mexican Americans: A randomized clinical trial. Journal of Clinical Child Adolescent Psychology, 38(5), 753759.

McCabe, K. M., Yeh, M., Garland, A. F., Lau, A. S., \& Chavez, G. (2005). The GANA program: A tailoring approach to adapting Parent-Child Interaction Therapy for Mexican Americans. Education and Treatment of Children, 28(2), 111-129.

McCabe, K., Yeh, M., Lau, A., \& Agote, C. B. (2012). Parent-Child Interaction Therapy for Mexican Americans: Results of a pilot randomized clinical trial at follow-up. Behavior Therapy, 43(3), 606-618.

McKay, M. M., Harrison, M. E., Gonzáles, J., Kim, L., \& Quintana, E. (2002). Multiple-family groups for urban children with conduct difficulties and their families. Psychiatric Services, 53(11), 1467-1468.

McNeil, C. B., Eyberg, S. M., Eisenstadt, T. H., Newcomb, K., \& Funderburk, B. W. (1991). Parent-Child Interaction Therapy with behavior problem children: Generalization of treatment effects to the school setting. Journal of Clinical Child Psychology, 20, 140-151.

McNeil, C. B., \& Hembree-Kigin, T. L. (2010). Parent-Child Interaction Therapy. New York, NY, US: Springer.

Nakamura, B. J., Ebesutani, C., Bernstein, A., \& Chorpita, B. F. (2009). A psychometric analysis of the Child Behavior Checklist DSM-oriented scales. Journal of Psychopathology \& Behavioral Assessment, 31, 178-189.

Nanninga, M., Jansen, D. E. M. C., Kazdin, A. E., Knorth, E. J., \& Reijneveld, S. A. (2016). Psychometric properties of the Barriers to Treatment Participation Scale-expectancies. Psychological Assessment, 28(8), 898-907. 
Nash, J. M., McCrory, D., Nicholson, R. A., \& Andrasik, F. (2005). Efficacy and effectiveness approaches in behavioral treatment trials. Headache: The Journal of Head and Face Pain, 45(5), 507-512.

National Institute of Drug Abuse [NIDA] (2018). Principles of drug addiction treatment: A research-based guide (3rd. ed.). Retrieved from: https://d14rmgtrwzf5a.cloudfront.net/sites/default/files/675-principles-of-drug-addictiontreatment-a-research-based-guide-third-edition.pdf

National Institute of Mental Health [NIMH] (2001). Blueprint for change: Research on child and adolescent mental health. Rockville, MD: U.S.

Niec, L. N., Acevedo-Polakovich, I. D., Abbenante-Honold, E., Christian, A. S., Barnett, M. L., Aguilar, G., \& Peer, S. O. (2014). Working together to solve disparities: Latina/o parents' contributions to the adaptation of a preventive intervention for childhood conduct problems. Psychological Services, 11(4), 410-420.

Nixon, R. D., Sweeney, L., Erickson, D. B., \& Touyz, S. W. (2003) Parent-Child Interaction Therapy: A comparison of standard and abbreviated treatments for oppositional defiant preschoolers. Journal of Consulting \& Clinical Psychology, 71(2), 251-260.

Noordraven, E. L., Wierdsma, A. I., Blanken, P., Bloemendaal, A. F. T., Staring, A. B. P., \& Mulder, C. L. (2017). Financial incentives for improving adherence to maintenance treatment in patients with psychotic disorders (money for medication): A multicenter, open-label, randomized controlled trial. The Lancet Psychiatry, 4(3), 199-207.

Owens, P. L., Hoagwood, K., Horowitz, S. M., Leaf, P. J., Poduska, J. M., Kellam, S. G., \& Ialongo, N. S. (2002). Barriers to children's mental health services. Journal of the American Academy of Child \& Adolescent Psychiatry, 41, 731-738. 
Padilla, A. M. (2002). Hispanic psychology: A 25-year retrospective look. Online Readings in Psychology and Culture, 3(1). https://doi.org/10.9707/2307-0919.1025

Pearl, E., Thieken, L., Olafson, E., Boat, B., Connelly, L., Barnes, J., \& Putnam, F. (2012). Effectiveness of community dissemination of Parent-Child Interaction Therapy. Psychological Trauma: Theory, Research, Practice, \& Policy, 4(2), 204-213.

Petry, N. M. (2000). A comprehensive guide for the application of incentive procedures in standard clinic settings. Drug and Alcohol Dependence, 58, 9-25.

Petry, N. M., Bickel, W. K., Tzanis, E., Taylor, R., Kubik, E., Foster, M., \& Hughes, M. E. (1998). A behavioral intervention for improving verbal behaviors of heroin addicts in a treatment clinic. Journal of Applied Behavioral Analysis, 31, 291-297.

Petry, N. M., Tedford, J., Austin, M., Nich, C., Carroll, K., \& Rounsaville, B. J. (2004). Prize reinforcement contingency management for treating cocaine users: How low can we go, and with whom? Addiction, 99, 349-360.

Pollastri, A. R., Pokrywa, M. L., Walsh, S. J., Kranzler, H. R., \& Gelernter, J. (2005). Incentive program decreases no-shows in nontreatment substance abuse research. Experimental \& Clinical Psychopharmacology, 13(4), 376-380.

Reid, M. J., Webster-Stratton, C., \& Hammond, M. (2003). Follow-up of children who received the incredible years intervention for oppositional defiant disorder: Maintenance and prediction of 2-year outcome. Behavior Therapy, 34, 471-491.

Reyno, S. M., \& McGrath, P. J. (2006). Predictors of parent training efficacy for child externalizing behavior problems - A meta-analytic review. Journal of Child Psychology and Psychiatry, 47(1), 99-111. 
Robbins, M. S., Liddle, H. A., Dakof, G. A., Turner, C. W., Alexander, J. F., \& Kogan, S. M. (2006). Adolescent and parent therapeutic alliances as predictors of dropout in Multidimensional Family Therapy. Journal of Family Psychology, 20(1), 108-116.

Robinson, E. A., \& Eyberg, S. M. (1981). The Dyadic Parent-Child Interaction Coding System: Standardization and validation. Journal of Consulting and Clinical Psychology, 49, 245250.

Rogers, C. P. (1951). Client-centered therapy. Boston: Houghton Mifflin.

Ros, R., Hernandez, J., Graziano, P. A., \& Bagner, D. M. (2016). Parent training for children with or at risk for developmental delay: The role of parental homework completion. Behavior Therapy, 47, 1-13.

Russell, M., Lang, M., \& Brett, B. (1987). Reducing dropout rates through improved intake procedures. Social Casework, 68, 421-425.

Ryan, R. M., \& Deci, E. L. (2000). Self-determination theory and the facilitation of intrinsic motivation, social development, and well-being. American Psychologist, 55, 68-78.

Sacks, R. M., Greene, J., Burke, R., \& Owen, E. C. (2015). Mental health care among lowincome pregnant women with depressive symptoms: Facilitators and barriers to care access and the effectiveness of financial incentives for increasing care. Administration and Policy in Mental Health, 42(4), 484-492.

Schuhmann, E. M., Foote, R. C., Eyberg, S. M., Boggs, S. R., \& Algina, J. A. (1998). Efficacy of Parent-Child Interaction Therapy: Interim report of a randomized trial with short-term maintenance. Journal of Clinical Child Psychology, 27, 34-45. 
Seligman, L. D., Ollendick, T. H., Langley, A. K., \& Baldacci, H. B. (2004). The utility of measures of child and adolescent anxiety: A meta-analytic review of the revised children's manifest anxiety scale, the state-trait anxiety inventory for children, and the child behavior checklist. Journal of Clinical Child and Adolescent Psychology, 33, 557565.

Silverman, K., Chutuape, M. A., Bigelow, G. E., \& Stitzer, M. L. (1996). Voucher-based reinforcement of attendance by unemployed methadone patients in a job skills training program. Drug and Alcohol Dependence, 41, 197-207.

Silverman, K., Chutuape, M. A., Bigelow, G. E., \& Stitzer, M. L. (1999). Voucher-based reinforcement of cocaine abstinence in treatment-resistant methadone patients: Effects of reinforcement magnitude. Psychopharmacology, 146, 128-138.

Singh, H., Janes, C. L., \& Schechtman, J. M. (1982). Problem children's treatment attrition and parents' perception of the diagnostic evaluation. Journal of Psychiatric Treatment Evaluation, 4, 257-263.

Sofronoff, K., Leslie, A., \& Brown, W. (2004). Parent management training and Asperger syndrome: A randomized controlled trial to evaluate a parent based intervention. Autism, 8(3), 301-317.

Stokes, J., McNeil, C. B., \& Wallace, N. M. (in press). Effectiveness of community-delivered Parent-Child Interaction Therapy compared to usual care. Child and Family Behavior Therapy. 
Stokes, J. O., Jent, J. F., Weinstein, A., Davis, E. M., Brown, T. M., Cruz, L., \& Wavering, H. (2016). Does practice make perfect? The relationship between self-reported treatment homework completion and parental skill acquisition and child behaviors. Behavior Therapy, 47(4), 538-549.

Stoolmiller, M., Duncan, T., Bank, L., \& Patterson, G. R. (1993). Some problems and solutions in the study of change: Significant patterns in client resistance. Journal of Consulting and Clinical Psychology, 61(6), 920-928.

Sukhodolsky, D. G., Smith, S. D., McCauley, S. A., Ibrahim, K., \& Piasecka, J. B. (2016). Behavioral interventions for anger, irritability, and aggression in children and adolescents. Journal of Child and Adolescent Psychopharmacology, 26, 58-64.

Tandon, M., Cardeli, E., \& Luby, J. (2009). Internalizing disorders in early childhood: A review of depressive and anxiety disorders. Child \& Adolescent Psychiatric Clinics of North America, 18(3), 593-610.

Timmer, S. G., Urquiza, A. J., Zebell, N. M., \& McGrath, J. M. (2005). Parent-Child Interaction Therapy: Application to maltreating parent-child dyads. Child Abuse \& Neglect, 29(7), $825-842$.

Timmer, S. G., Zebell, N. M., Culver, M. A., \& Urquiza, A. J. (2010). Efficacy of adjunct inhome coaching to improve outcomes in Parent-Child Interaction Therapy. Research on Social Work Practice, 20, 36-45.

U.S. Public Health Service (2001). Report of the Surgeon General's Conference on Children's Mental Health: A National Action Agenda. Washington, DC: Department of Health and Human Services. 
Van Horn, D. H. A., Drapkin, M., Ivey, M., Thomas, T., Domis, S. W., Abdalla, O., Herd, D. H., \& McKay, J. R. (2011). Voucher incentives increase treatment participation in telephonebased continuing care for cocaine dependence. Drug and Alcohol Dependence, 114(2-3), $225-228$.

Vasquez, M. J. T. (2007). Sometimes a taco is just a taco! Professional Psychology: Research and Practice, 38(4), 401-410.

Ward, M A., Theule, J., \& Cheung, K. (2016). Parent-Child Interaction Therapy for child disruptive behavior disorders: A meta-analysis. Child \& Youth Care Forum, 45(5), 675690.

Webb, H. J., Thomas, R., McGregor, L., Avdagic, E., \& Zimmer-Gembeck, M. J. (2017). An evaluation of Parent-Child Interaction Therapy with and without motivational enhancement to reduce attrition. Journal of Clinical Child \& Adolescent Psychology, 46(4), 537-550.

Werba, B. E., Eyberg, S. M., Boggs, S. R., \& Algina, J. (2006). Predicting outcome in ParentChild Interaction Therapy: Success and attrition. Behavior Modification, 30(5), 618-646.

Whiteside-Mansell, L., Ayoub, C., McKelvey, L., Faldowski, R. A., Hart, A., \& Shears, J. (2007). Parenting stress of low-income parents of toddlers and preschoolers: Psychometric properties of a short form of the Parenting Stress Index. Parenting: Science and Practice, 7(1), 27-56.

Zisser, A., \& Eyberg, S. M. (2010). Parent-Child Interaction Therapy and the treatment of disruptive behavior disorders. In J. R. Weisz, \& A. E. Kazdin (Eds.), Evidence-based psychotherapies for children and adolescents, second edition (pp. 179-193). New York: Guilford Press. 
Table 1

Therapist Demographics

Demographic $\quad M(S D) \quad n(\%)$

Sex

Female

$9(100 \%)$

Age (years)

$36.67(7.62)$

Ethnicity

Hispanic

$5(55.6 \%)$

Caucasian

$2(22.2 \%)$

Mixed

$2(22.2 \%)$

Primary Language

Spanish

$1(11.1 \%)$

English

$8(88.9 \%)$

Secondary Language

Spanish

$4(44.4 \%)$

English

$1(11.1 \%)$

None

$4(44.4 \%)$

Education

Master's degree

$6(66.7 \%)$

Doctorate

$1(11.1 \%)$

Unspecified graduate school

$2(22.2 \%)$

Years in Clinical Work

$8.33(5.68)$

Years Conducting PCIT

$4.89(3.95)$

Number of PCIT Cases Total

$99.89(93.13)$

Number PCIT Graduated Cases 44.00 (39.42)

Notes. $N=9$; PCIT = Parent-Child Interaction Therapy. 
Table 2a

Between-group Differences on Continuous Family Demographic Variables

\begin{tabular}{|c|c|c|c|c|c|c|c|}
\hline \multirow{3}{*}{ Demographic } & \multicolumn{4}{|c|}{ Group } & \multirow[b]{3}{*}{$\underline{t}$} & \multirow[b]{3}{*}{$\underline{p}$} & \multirow[b]{3}{*}{ Cohen's $d$} \\
\hline & \multicolumn{2}{|r|}{ Incentive } & \multicolumn{2}{|r|}{ Nonincentive } & & & \\
\hline & $\underline{n}$ & $\underline{M(S D)}$ & $\underline{n}$ & $\underline{M(S D)}$ & & & \\
\hline \multicolumn{8}{|l|}{ Caregiver } \\
\hline Age (years) & 49 & 35.84 (7.97) & 30 & $34.37(8.61)$ & .77 & .44 & .18 \\
\hline Income & 42 & $22,514(21,992)$ & 29 & $33,562(25,720)$ & -1.50 & .14 & .37 \\
\hline \multicolumn{8}{|l|}{ Child } \\
\hline Age (years) & 50 & $3.76(1.20)$ & 34 & $3.74(.90)$ & .11 & .92 & .02 \\
\hline
\end{tabular}

Table $2 b$

Between-group Differences on Categorical Family Demographic Variables: Caregiver Role

\begin{tabular}{lcc}
\hline & \multicolumn{2}{c}{ Group } \\
\cline { 2 - 3 } Role & Incentive & Nonincentive \\
\hline Mother & $n(\%)$ & $n(\%)$ \\
Father & $42(84.0 \%)$ & $26(76.5 \%)$ \\
Maternal Grandparent & $2(4.0 \%)$ & $1(2.9 \%)$ \\
Paternal Grandparent & $1(2.0 \%)$ & $1(2.9 \%)$ \\
Step Parent & $2(4.0 \%)$ & $1(2.9 \%)$ \\
Adoptive/Foster Parent & $2(4.0 \%)$ & $0(0.0 \%)$ \\
\hline Total & $50(100.0 \%)$ & $5(14.7 \%)$ \\
\hline
\end{tabular}

Notes. $\chi^{2}(5)=4.50, p=.48, \phi=.23$ (small). Number in parentheses indicates column percentages. 
Table 2c

Between-group Differences on Categorical Family Demographic Variables: Caregiver Sex

\begin{tabular}{lcc} 
& \multicolumn{2}{c}{ Group } \\
\cline { 2 - 3 } & Incentive & Nonincentive \\
Sex & $n(\%)$ & $n(\%)$ \\
\hline Female & $47(94.0 \%)$ & $33(97.1 \%)$ \\
Male & $3(6.0 \%)$ & $1(2.9 \%)$ \\
\hline
\end{tabular}

Total $\quad 50(100.0 \%) \quad 34(100.0 \%)$

Notes. $\chi^{2}(1)=.42, p=.52, \phi=.07$ (none/small). Number in parentheses indicates column percentages.

Table $2 \mathrm{~d}$

Between-group Differences on Categorical Family Demographic Variables: Caregiver Ethnicity

\begin{tabular}{lcc}
\hline & \multicolumn{2}{c}{ Group } \\
\cline { 2 - 3 } Ethnicity & Incentive & Nonincentive \\
\hline Hispanic & $n(\%)$ & $n(\%)$ \\
African American & $23(66.0 \%)$ & $20(58.8 \%)$ \\
Caucasian & $11(22.0 \%)$ & $1(2.9 \%)$ \\
Mixed & $4(8.0 \%)$ & $2(5.9 \%)$ \\
\hline Total & $50(100.0 \%)$ & $34(100.0 \%)$
\end{tabular}

Notes. $\chi^{2}(3)=1.18, p=.76, \phi=.12$ (small). Number in parentheses indicates column percentages. 
Table $2 \mathrm{e}$

Between-group Differences on Categorical Family Demographic Variables: Caregiver Mental Health History

\begin{tabular}{lcc}
\hline & \multicolumn{2}{c}{ Group } \\
\cline { 2 - 3 } Mental Health History & $n(\%)$ & Nonincentive \\
\hline Yes & $13(26.0 \%)$ & $13(38.2 \%)$ \\
No & $37(74.0 \%)$ & $21(61.8 \%)$ \\
\hline Total & $50(100.0 \%)$ & $34(100.0 \%)$
\end{tabular}

Notes. $\chi^{2}(1)=1.42, p=.23, \phi=.13$ (small). Number in parentheses indicates column percentages.

Table $2 \mathrm{f}$

Between-group Differences on Categorical Family Demographic Variables: Child Sex

\begin{tabular}{lcc} 
& \multicolumn{2}{c}{ Group } \\
\cline { 2 - 3 } Sex & Incentive & Nonincentive \\
& $n(\%)$ & $n(\%)$ \\
Female & $20(40.0 \%)$ & $8(23.5 \%)$ \\
Male & $30(60.0 \%)$ & $26(76.5 \%)$ \\
\hline Total & $50(100.0 \%)$ & $34(100.0 \%)$ \\
\hline
\end{tabular}

Notes. $\chi^{2}(1)=2.47, p=.12, \phi=.17$ (small). Number in parentheses indicates column percentages. 
Table $2 \mathrm{~g}$

Between-group Differences on Categorical Family Demographic Variables: Child Ethnicity

\begin{tabular}{lcc}
\hline & \multicolumn{2}{c}{ Group } \\
\cline { 2 - 3 } Ethnicity & Incentive & Nonincentive \\
\hline Hispanic & $n(\%)$ & $n(\%)$ \\
African American & $33(67.3 \%)$ & $18(52.9 \%)$ \\
Caucasian & $2(4.1 \%)$ & $1(2.9 \%)$ \\
Mixed & $6(12.2 \%)$ & $4(11.8 \%)$ \\
\hline Total & $8(16.3 \%)$ & $11(32.4 \%)$ \\
\hline
\end{tabular}

Notes. $\chi^{2}(3)=3.01, p=.39, \phi=.19$ (small). Number in parentheses indicates column percentages.

Table $2 \mathrm{~h}$

Between-group Differences on Categorical Family Demographic Variables: Child Mental Health History

Group

Incentive Nonincentive

Mental Health History $\quad n(\%) \quad n(\%)$

\begin{tabular}{lcc}
\hline Yes & $19(38.0 \%)$ & $11(32.4 \%)$ \\
No & $31(62.0 \%)$ & $23(67.6 \%)$ \\
\hline Total & $50(100.0 \%)$ & $34(100.0 \%)$
\end{tabular}

Notes. $\chi^{2}(1)=.28, p=.60, \phi=.06$ (none/small). Number in parentheses indicates column percentages. 
Table 3

Between-group Differences in Number of Sessions and Length of Time to CDI Mastery

\begin{tabular}{|c|c|c|c|c|c|}
\hline \multirow{2}{*}{$\begin{array}{l}\text { Length of Time to } \\
\text { CDI Mastery }\end{array}$} & \multicolumn{2}{|c|}{ Group } & & & \\
\hline & Incentive $^{\mathrm{a}}$ & Nonincentive $^{b}$ & & & \\
\hline & $\underline{M(S D)}$ & $\underline{M(S D)}$ & $\underline{t}$ & $\underline{p}$ & $\underline{\text { Cohen's } d}$ \\
\hline Number of sessions & $6.44(3.04)$ & $6.23(2.86)$ & .23 & .82 & .07 \\
\hline Length (weeks) & $13.10(7.51)$ & $13.87(6.98)$ & -.35 & .73 & .11 \\
\hline
\end{tabular}

Notes. $n^{\mathrm{a}}=25, n^{\mathrm{b}}=21 . \mathrm{CDI}=$ Child-Directed Interaction. 
Table 4

Between-group Differences in Family Termination Prior to CDI Mastery

\begin{tabular}{lcc}
\hline & \multicolumn{2}{c}{ Group } \\
\cline { 2 - 3 } & Incentive & Nonincentive \\
Termination Before CDI Mastery & $n(\%)$ & $n(\%)$ \\
\hline Yes & $26(52.0 \%)$ & $13(38.2 \%)$ \\
No & $24(48.0 \%)$ & $21(61.8 \%)$ \\
\hline Total & $50(100.0 \%)$ & $34(100.0 \%)$
\end{tabular}

Notes. $\chi^{2}(1)=1.54, p=.21, \phi=.14$ (small). CDI = Child-Directed Interaction. Number in parentheses indicates column percentages. 
Table 5

Between-group Differences in Treatment Adherence Components

\begin{tabular}{|c|c|c|c|c|c|}
\hline & \multicolumn{2}{|c|}{ Group } & & & \\
\hline & Incentive $^{\mathrm{a}}$ & Nonincentive $^{b}$ & & & \\
\hline & $\underline{M(S D)}$ & $M(S D)$ & $\underline{t}$ & $\underline{p}$ & Cohen's $d$ \\
\hline Percentage No-shows & $.11(.15)$ & $.16(.18)$ & -1.79 & .08 & .41 \\
\hline Percentage Cancels & $.25(.18)$ & $.21(.15)$ & 1.01 & .31 & .19 \\
\hline Percentage Late Sessions & $.04(.06)$ & $.06(.14)$ & -1.31 & .20 & .18 \\
\hline \multicolumn{6}{|l|}{ Homework } \\
\hline Paper & $1.69(1.67)$ & $2.01(1.91)$ & -.69 & .49 & .15 \\
\hline Audio & $1.15(1.37)$ & $1.14(1.52)$ & -.02 & .99 & .00 \\
\hline
\end{tabular}

Notes. $n^{\mathrm{a}}=50, n^{\mathrm{b}}=34$ 
Table 6

Between-group Differences in Family Attainment of CDI Mastery

\begin{tabular}{lcc}
\hline & \multicolumn{2}{c}{ Group } \\
\cline { 2 - 3 } Reached CDI Mastery & Incentive & Nonincentive \\
\hline Yes & $n(\%)$ & $n(\%)$ \\
No & $24(48.0 \%)$ & $21(61.8 \%)$ \\
\hline Total & $26(52.0 \%)$ & $13(38.2 \%)$ \\
\hline
\end{tabular}

Notes. $\chi^{2}(1)=1.54, p=.21, \phi=.14$ (small). CDI $=$ Child-Directed Interaction. Number in parentheses indicates column percentages. 
Table 7

Between-group Differences in Family Attainment of PDI Mastery/Graduation

\begin{tabular}{lcc}
\hline \multirow{2}{*}{$\begin{array}{c}\text { Reached PDI } \\
\text { Mastery/Graduation }\end{array}$} & $n(\%)$ & $n(\%)$ \\
\cline { 2 - 3 } & Incentive & Nonincentive \\
\hline Yes & $19(38.0 \%)$ & $15(44.1 \%)$ \\
No & $31(62.0 \%)$ & $19(55.9 \%)$ \\
\hline Total & $50(100 \%)$ & $34(100 \%)$
\end{tabular}

$\begin{array}{lll}\text { Total } & 50(100 \%) & 34(100 \%)\end{array}$

Notes. $\chi^{2}(1)=1.31, p=.58, \phi=.06$ (none/small). PDI $=$ Parent-Directed Interaction. Number in parentheses indicates column percentages. 
Table 8a

Between-group Mean Comparisons of Child Behavior Problems from First to Last Attended Session-T-Scores

\begin{tabular}{lcc}
\hline & \multicolumn{2}{c}{ Group } \\
\hline \multirow{2}{*}{ Time Point } & Incentive $^{\mathrm{a}}$ & Nonincentive $^{\mathrm{b}}$ \\
\cline { 2 - 3 } First session & $66.64(9.17)$ & $\underline{M(S D)}$ \\
Last session & $57.50(12.20)$ & $67.48(9.26)$ \\
\hline
\end{tabular}

Notes. $n^{\mathrm{a}}=50, n^{\mathrm{b}}=33 . F(1,81)=.38, p=.54, \eta^{2}=00$. Outcomes are T-scores for the Eyberg Child Behavior Inventory, Intensity scale.

Table $8 b$

Between-group Mean Comparisons of Child Behavior Problems from First to Last Attended Session-Raw Scores

\begin{tabular}{lcc}
\hline & \multicolumn{2}{c}{ Group } \\
\hline \multirow{2}{*}{ Time Point } & Incentive $^{\mathrm{a}}$ & Nonincentive $^{\mathrm{b}}$ \\
\cline { 2 - 3 } First session & $155.16(32.09)$ & $\underline{M(S D)}$ \\
Last session & $122.98(42.93)$ & $158.03(32.27)$ \\
\end{tabular}

Notes. $n^{\mathrm{a}}=50, n^{\mathrm{b}}=33$. Outcomes are raw scores for the Eyberg Child Behavior Inventory, Intensity scale. 
Table 9

Between-group Mean Comparisons of Externalizing Child Behaviors throughout Treatment

\begin{tabular}{lcc}
\hline & \multicolumn{2}{c}{ Group } \\
\hline \multirow{2}{*}{ Time Point } & Incentive $^{\mathrm{a}}$ & Nonincentive $^{\mathrm{b}}$ \\
\cline { 2 - 3 } Pre & $\frac{M(S D)}{M(S D)}$ \\
Mid & $29.00(9.89)$ & $25.36(9.42)$ \\
Post & $22.18(11.17)$ & $20.14(9.40)$ \\
\hline
\end{tabular}

Notes. $n^{\mathrm{a}}=17, n^{\mathrm{b}}=14 . F(2,28)=5.54, p=.90, \eta^{2}=.03$. Outcomes are for the Child Behavior Checklist, Externalizing scale. 
Table 10

Between-group Mean Comparisons of Internalizing Child Behaviors throughout Treatment

\begin{tabular}{lcc}
\hline & \multicolumn{2}{c}{ Group } \\
\hline \multirow{2}{*}{ Time Point } & Incentive $^{\mathrm{a}}$ & Nonincentive $^{\mathrm{b}}$ \\
\cline { 2 - 3 } Pre & $18.06(9.92)$ & $\frac{M(S D)}{M(S D)}$ \\
Mid & $15.24(12.62)$ & $14.57(9.41)$ \\
Post & $11.00(11.59)$ & $7.64(10.71)$ \\
\hline
\end{tabular}

Notes. $n^{\mathrm{a}}=17, n^{\mathrm{b}}=14 . F(2,28)=.002, p=1.00, \eta^{2}=.04$. Outcomes are for the Child Behavior Checklist, Internalizing scale. 
Table 11

Between-group Mean Comparisons of Parenting Stress throughout Treatment

\begin{tabular}{lcc} 
& \multicolumn{2}{c}{ Group } \\
\hline \multirow{2}{*}{ Time Point } & Incentive $^{\mathrm{a}}$ & Nonincentive $^{\mathrm{b}}$ \\
\cline { 2 - 3 } Pre & $\frac{M(S D)}{M(S D)}$ \\
Mid & $89.72(18.67)$ & $102.00(22.40)$ \\
Post & $81.67(22.22)$ & $89.87(18.23)$ \\
& $68.06(20.73)$ & $69.27(20.43)$
\end{tabular}

Notes. $n^{\mathrm{a}}=17, n^{\mathrm{b}}=14 . F(2,28)=1.09, p=.35, \eta^{2}=.04$. Outcomes are for the Parenting Stress Index: Short Form, Total Stress scale. 
Table 12

Between-group Comparisons of Cost Analysis for the Community-Based Agency

\section{Group}

\begin{tabular}{|c|c|c|c|c|c|}
\hline & Incentive $^{\mathrm{a}}$ & Nonincentive $^{b}$ & & & \\
\hline & $\underline{M(S D)}$ & $\underline{M(S D)}$ & $\underline{t}$ & $p$ & Cohen's $d$ \\
\hline Cost Analysis & $1,489(2,176)$ & $1,623(2,043)$ & -.29 & .78 & .06 \\
\hline
\end{tabular}

Notes. $n^{\mathrm{a}}=50, n^{\mathrm{b}}=34$. 
Table 13

Binary Logistic Regression for Exploratory Variables Contributing to Treatment Termination

\begin{tabular}{|c|c|c|c|c|c|c|c|}
\hline Source & $n$ & $\beta$ & $S E$ & Wald $\chi^{2}$ & $p$ & $O R$ & $95 \%$ CI OR \\
\hline \multicolumn{8}{|l|}{ Group Status } \\
\hline Incentive Group & 41 & & & & & & \\
\hline Nonincentive Group & 26 & .93 & .74 & 1.57 & .21 & 2.53 & {$[.59,10.73]$} \\
\hline Caregiver Age & 79 & -.06 & .05 & 1.56 & .21 & .94 & {$[.86,1.04]$} \\
\hline Family Income & 74 & -.38 & .72 & .28 & .60 & .68 & {$[.17,2.80]$} \\
\hline \multicolumn{8}{|l|}{ Caregiver Ethnicity } \\
\hline Hispanic & 44 & & & 5.52 & .14 & & \\
\hline African American & 2 & 2.19 & 1.50 & 2.13 & .15 & 8.93 & {$[.47,169.43]$} \\
\hline Caucasian & 16 & 2.05 & 2.20 & .86 & .35 & 7.73 & {$[.10,579.93]$} \\
\hline Mixed & 5 & 3.77 & 1.72 & 4.79 & $.03 *$ & 43.52 & {$[1.48,1276.26]$} \\
\hline \multicolumn{8}{|l|}{ Caregiver MH Hx } \\
\hline Previous MH Hx & 21 & & & & & & \\
\hline No Previous MH Hx & 46 & .44 & .76 & .34 & .56 & 1.55 & {$[.35,6.84]$} \\
\hline PSI:SF Total Stress & 83 & -.02 & .02 & 1.88 & .17 & .98 & {$[.95,1.01]$} \\
\hline $\begin{array}{l}\text { DPICS Positive } \\
\text { Parenting Composite }\end{array}$ & 82 & .02 & .05 & .11 & .74 & 1.02 & {$[.92,1.12]$} \\
\hline $\begin{array}{l}\text { DPICS Negative } \\
\text { Parenting Composite }\end{array}$ & 82 & -.02 & .02 & .65 & .42 & .98 & {$[.94,1.03]$} \\
\hline CBCL Internalizing & 81 & .15 & .05 & 8.43 & $.00 *$ & 1.17 & {$[1.05,1.29]$} \\
\hline CBCL Externalizing & 81 & -.08 & .06 & 1.84 & .18 & .93 & {$[.83,1.04]$} \\
\hline
\end{tabular}

Notes. CBCL $=$ Child Behavior Checklist; DPICS $=$ Dyadic Parent-Child Interaction Coding System; MH Hx = Mental Health History; PSI:SF = Parenting Stress Index: Short Form.

Incentive Group uses incentives as the reference group (vs. nonincentive families). Hispanic 
families are the reference group in the Caregiver Ethnicity variable. Caregivers with a mental health history are the reference group compared to those without. 
Table 14

Between-group Differences for CDI Mastery Status of Barriers to Treatment Participation Scale Items at CDI Coach 3

\section{Status}

\begin{tabular}{|c|c|c|c|c|c|}
\hline & $\begin{array}{l}\text { Terminated Prior to } \\
\text { CDI Mastery }\end{array}$ & Reached Mastery ${ }^{\mathrm{b}}$ & & & \\
\hline & $\underline{M(S D)}$ & $\underline{M(S D)}$ & $\underline{t}$ & $p$ & Cohen's $d$ \\
\hline Q1 - Child session refusal & $1.17(.44)$ & $1.00(.00)$ & 2.56 & .01 & -- \\
\hline Q28 - Child new problems & $1.40(.66)$ & $1.06(.24)$ & 2.89 & $<.01$ & .69 \\
\hline $\begin{array}{l}\text { Q40 - Disagreement with } \\
\text { partner about treatment }\end{array}$ & $1.26(.73)$ & $1.00(.00)$ & 2.65 & .01 & -- \\
\hline
\end{tabular}

Notes. $n^{\mathrm{a}}=17, n^{\mathrm{b}}=42 . \mathrm{CDI}=$ Child-Directed Interaction. 
Table 15

Between-group Differences for Graduation Status of Barriers to Treatment Participation Scale Items at Midtreatment

\begin{tabular}{|c|c|c|c|c|c|}
\hline \multicolumn{6}{|c|}{ Graduation Status } \\
\hline & $\begin{array}{c}\text { Terminated Prior to } \\
\text { Graduation }^{\mathrm{a}}\end{array}$ & Graduated $^{\mathrm{b}}$ & & & \\
\hline & $\underline{M(S D)}$ & $\underline{M(S D)}$ & $\underline{t}$ & $\underline{p}$ & Cohen's $d$ \\
\hline Q16 - Parental sickness & $2.13(.83)$ & $1.27(.45)$ & -3.57 & $<.01$ & 1.22 \\
\hline
\end{tabular}

Notes. $n^{\mathrm{a}}=32, n^{\mathrm{b}}=8$. 
Table 16

Ethnicity Differences on CDI Mastery Attainment

\begin{tabular}{lcccc}
\hline & \multicolumn{4}{c}{ Ethnicity } \\
\cline { 2 - 5 } CDI Mastery & Hispanic & African American & Caucasian & Mixed \\
& $n(\%)$ & $n(\%)$ & $n(\%)$ & $n(\%)$ \\
\hline Reached mastery & $28(52.8 \%)$ & $2(66.7 \%)$ & $11(50.0 \%)$ & $4(66.7 \%)$ \\
Did not reach mastery & $25(47.2 \%)$ & $1(33.3 \%)$ & $11(50.0 \%)$ & $2(33.3 \%)$ \\
\hline Total & $53(100.0 \%)$ & $3(100.0 \%)$ & $22(100.0 \%)$ & $6(100.0 \%)$
\end{tabular}

Notes. $\chi(3)=0.75, p=.86, \phi=.09$ (none/small). CDI $=$ Child-Directed Interaction. Number in parentheses indicates column percentages. 
Table 17

Ethnicity Differences on PDI/Graduation Attainment

\begin{tabular}{lcccc}
\hline & \multicolumn{3}{c}{ Ethnicity } \\
\cline { 2 - 4 } & Hispanic & African American & Caucasian & Mixed \\
Graduation Status & $n(\%)$ & $n(\%)$ & $n(\%)$ & $n(\%)$ \\
\hline Graduated & $22(41.5 \%)$ & $1(33.3 \%)$ & $7(31.8 \%)$ & $4(66.7 \%)$ \\
Did not graduate & $31(58.5 \%)$ & $2(66.7 \%)$ & $15(68.2 \%)$ & $2(33.3 \%)$ \\
\hline Total & $53(100.0 \%)$ & $3(100.0 \%)$ & $22(100.0 \%)$ & $6(100.0 \%)$ \\
\hline
\end{tabular}

Notes. $\chi(3)=2.48, p=.48, \phi=.17$ (small). PDI = Parent-Directed Interaction. Number in parentheses indicates column percentages. 
Table 18a

Ethnicity Mean Comparisons of Child Behavior Problems from First to Last Attended SessionT-Scores

\section{Ethnicity}

\begin{tabular}{lcc}
\hline & Hispanic $^{\mathrm{a}}$ & Non-Hispanic $^{\mathrm{b}}$ \\
\cline { 2 - 3 } Time Point & $\underline{M(S D)}$ & $\underline{M(S D)}$ \\
First session & $66.45(9.83)$ & $67.90(7.93)$ \\
Last session & $55.55(12.29)$ & $60.13(13.24)$
\end{tabular}

Notes. $n^{\mathrm{a}}=53, n^{\mathrm{b}}=30 . F(1,81)=.70, p=.56, \eta^{2}=.00$. Outcomes are T-scores for the Eyberg Child Behavior Inventory, Intensity scale. Non-Hispanic = African American, Caucasian, mixed ethnicities.

Table $18 \mathrm{~b}$

Ethnicity Mean Comparisons of Child Behavior Problems from First to Last Attended SessionRaw Scores

\begin{tabular}{lcc}
\hline & \multicolumn{2}{c}{ Ethnicity } \\
\hline & Hispanic $^{\mathrm{a}}$ & Non-Hispanic $^{\mathrm{b}}$ \\
Time Point & $\underline{M(S D)}$ & $\underline{M(S D)}$ \\
\cline { 2 - 3 } First session & $154.60(34.39)$ & $159.30(27.56)$ \\
Last session & $116.21(43.18)$ & $132.03(46.55)$
\end{tabular}

Notes. $n^{\mathrm{a}}=53, n^{\mathrm{b}}=30$. Outcomes are raw scores for the Eyberg Child Behavior Inventory, Intensity scale. Non-Hispanic $=$ African American, Caucasian, mixed ethnicities. 
Table 19

Ethnicity Differences for Therapy Attitude Inventory and Barriers to Treatment Participation Scale Items at CDI Coach 3

\section{Ethnicity}

\begin{tabular}{llllll}
\cline { 2 - 4 } & Hispanic $^{\mathrm{a}}$ & Non-Hispanic $^{\mathrm{b}}$ & & \\
\hline & $\underline{M(S D)}$ & $\underline{M(S D)}$ & $\underline{t}$ & $\underline{p}$ & $\underline{\text { Cohen's } d}$
\end{tabular}

\section{$\underline{\text { TAI }}$}

Q8 - Treatment helped with other

problems

\section{$\underline{\text { BTPS }}$}

Q1 - Child session refusal

Q14 - Experienced lots of stress

Q15 - Lost interest in coming to treatment

Competing Activities/Life Stressors Scale
$1.19(.46)$

$1.00(.00)$

$2.58 \quad .01$

$2.95(1.43)$

$-2.08 \quad .04$

$1.18(.39)$

$-2.82 \quad .04$

$1.00(.00)$

$25.62(5.46)$

Notes. $n^{\mathrm{a}}=37, n^{\mathrm{b}}=22$. BTPS $=$ Barriers to Treatment Participation Scale; TAI $=$ Therapy Attitude Inventory. Non-Hispanic = African American, Caucasian, mixed ethnicities. 


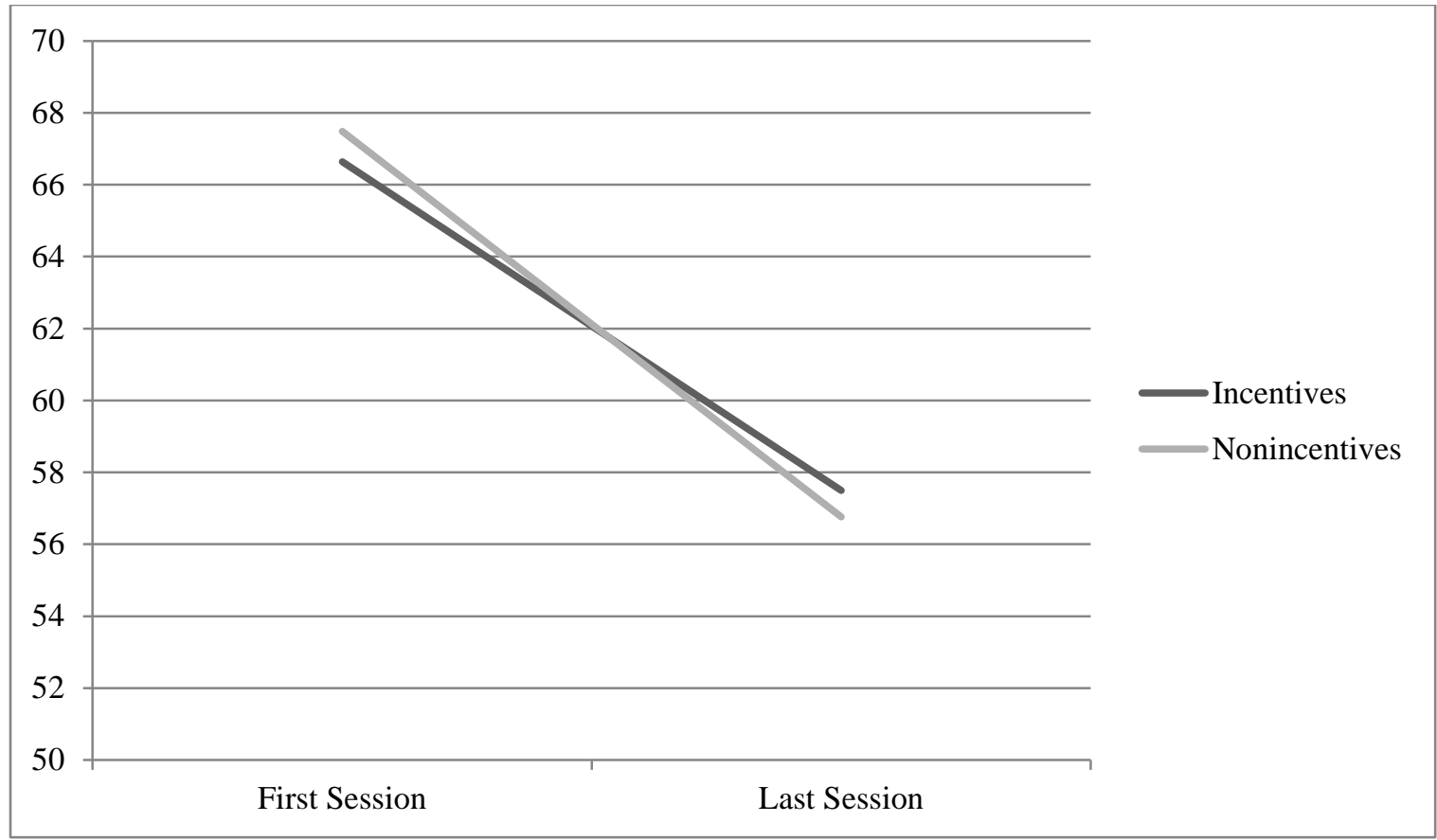

Figure 1. Eyberg Child Behavior Inventory - Intensity scale T-scores from first to last session attended for incentive and nonincentive groups. 


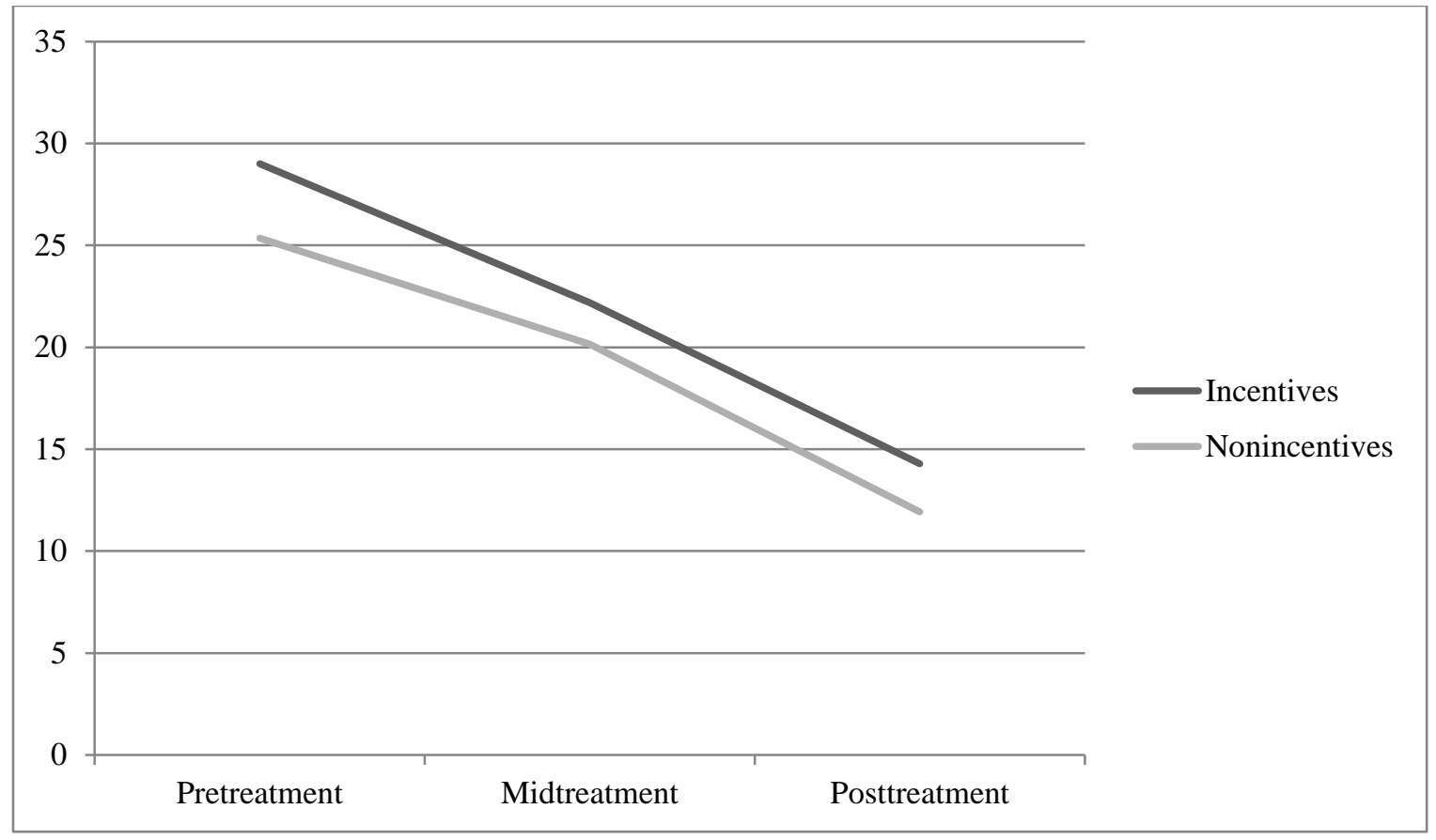

Figure 2. Child Behavior Checklist - Externalizing scale scores across treatment for incentive and nonincentive groups. 


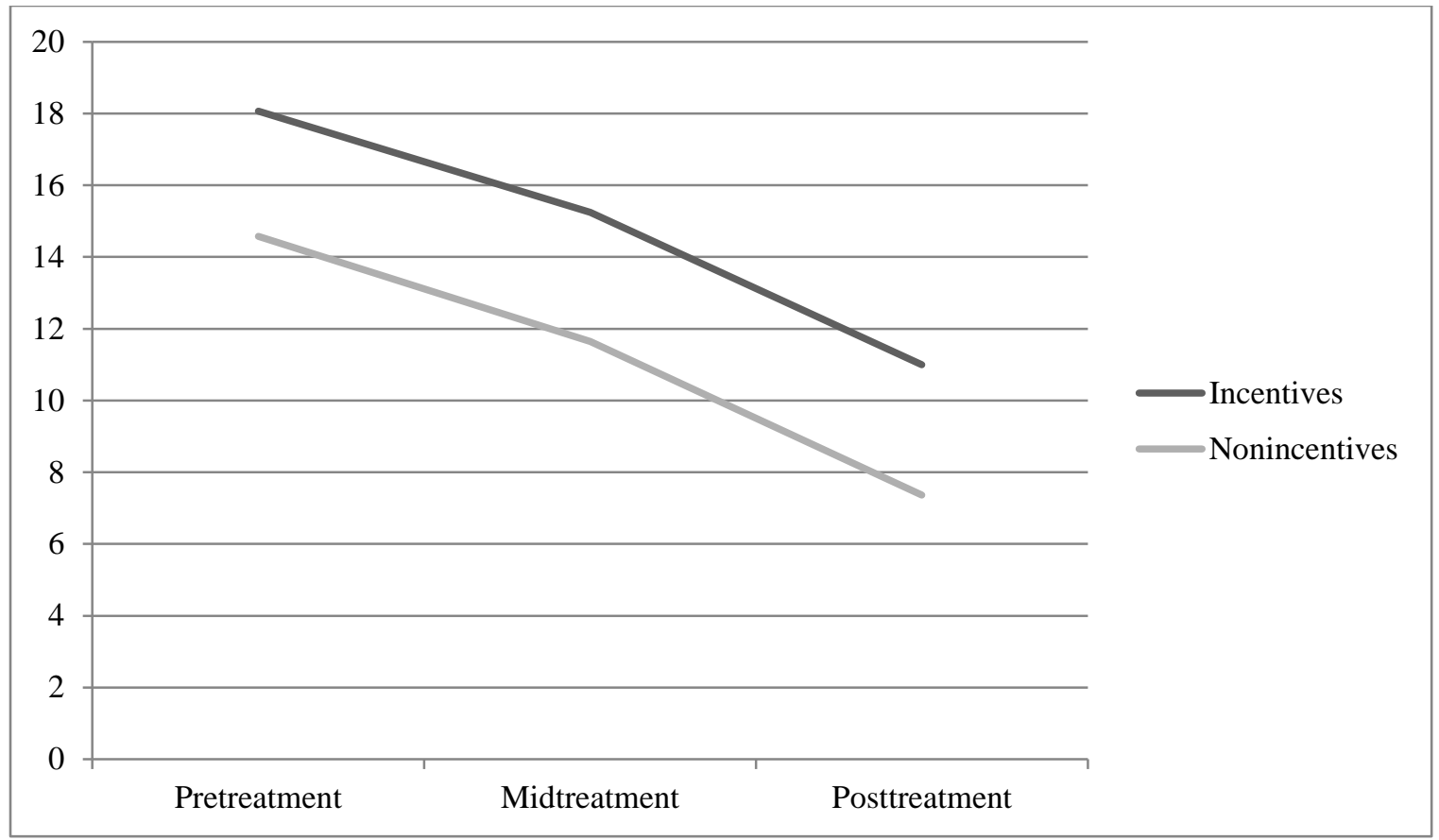

Figure 3. Child Behavior Checklist - Internalizing scale scores across treatment for incentive and nonincentive groups. 


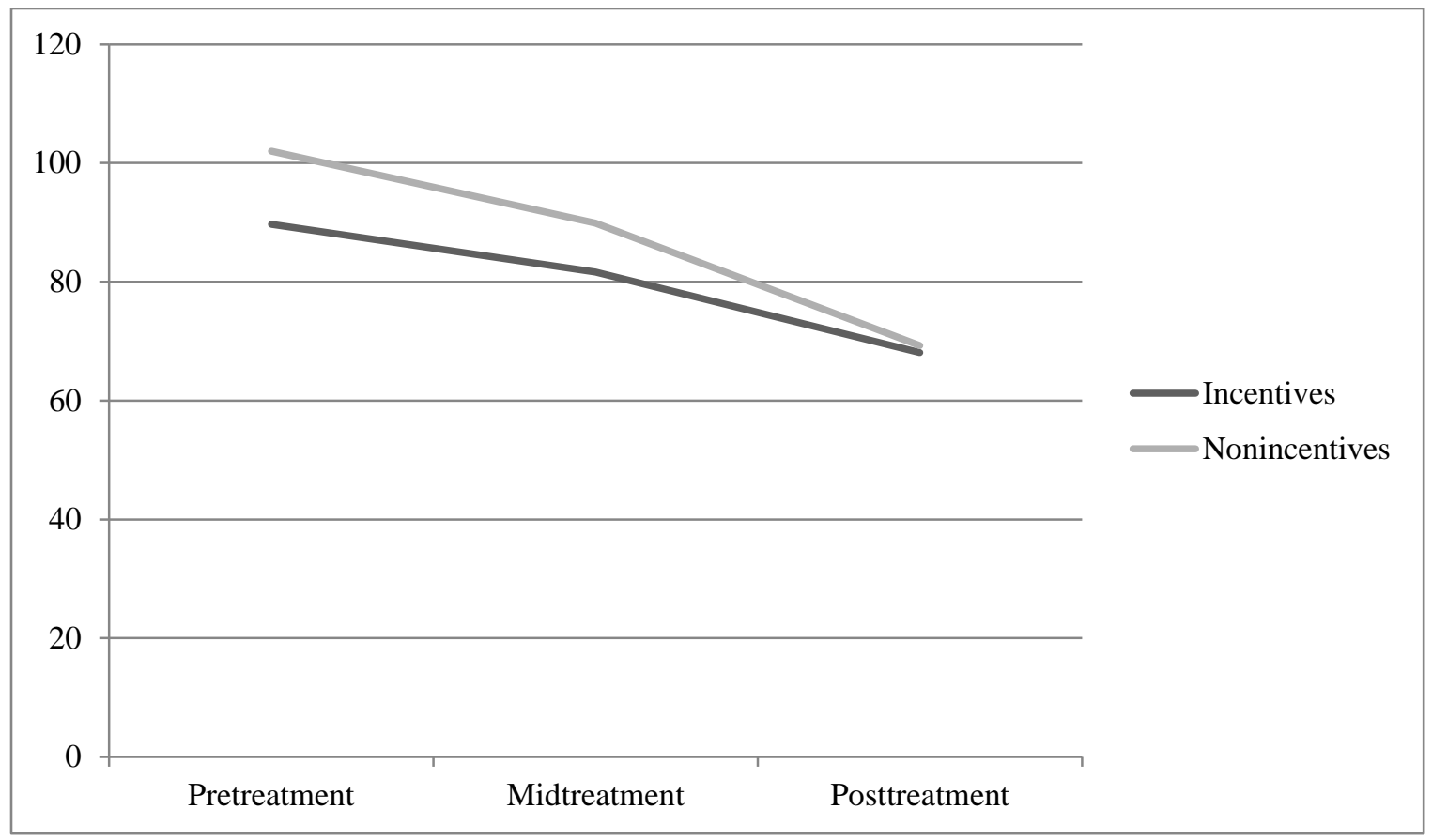

Figure 4. Parenting Stress Index: Short Form - Total Stress scale scores across treatment for incentive and nonincentive groups. 


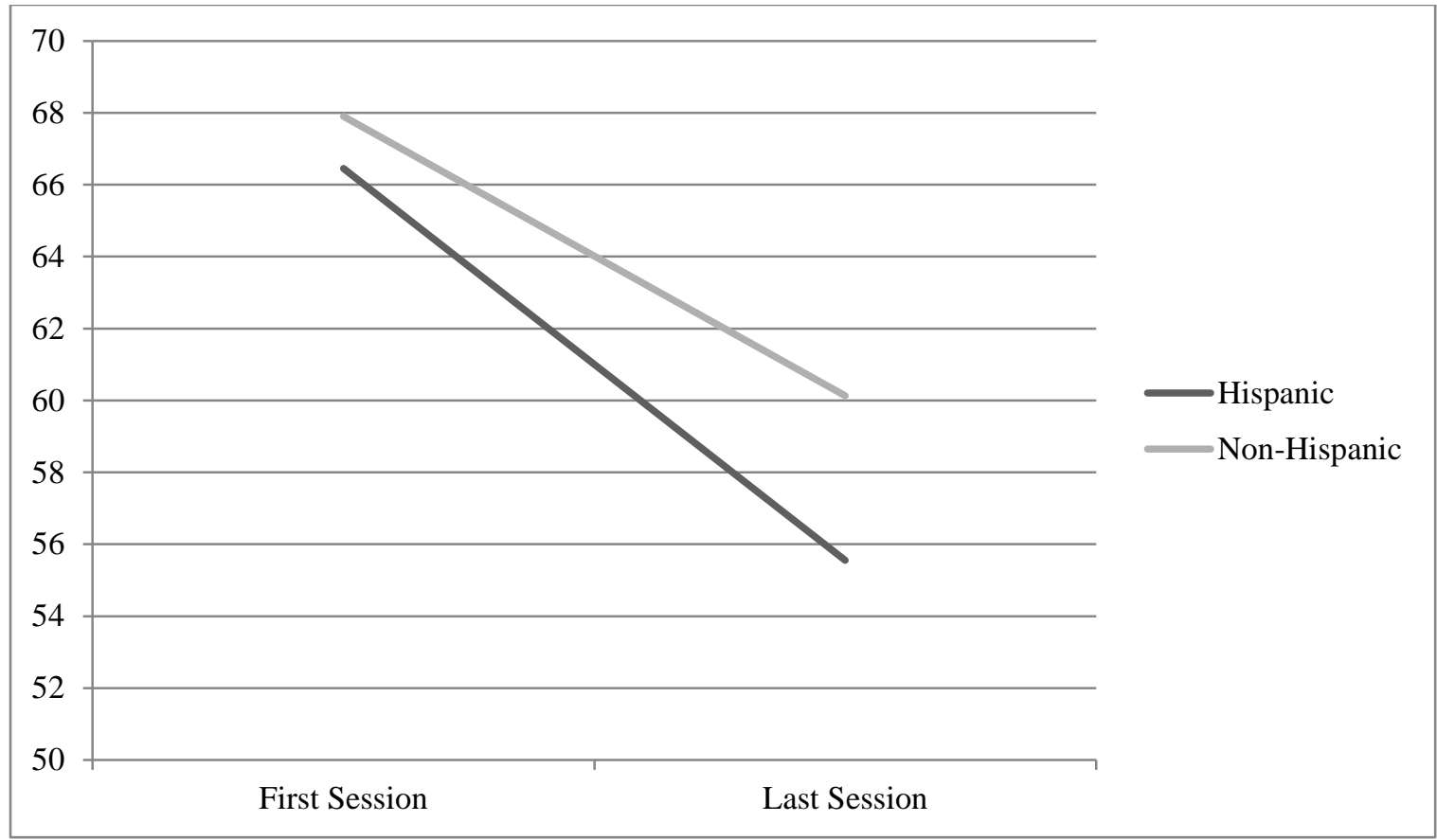

Figure 5. Eyberg Child Behavior Inventory - Intensity scale T-scores from first to last session attended for Hispanic and non-Hispanic families. Non-Hispanic = African American, Caucasian, mixed ethnicities. 
Appendix A

Family Demographic Form 
Therapist Number

\section{Family Demographic Form}

Family ID

Caregiver (Primary)

Relationship to child

Sex: $M / F$

Age

Ethnicity

Income (per year)

Mental Health History (Previous Diagnoses, Therapy Visits, etc.):

Child

Ethnicity

Sex: $M / F$

Age

Mental Health History (Previous Diagnoses, Therapy Visits, etc.):

CBCL: Pre: In

Ex

Mid: In

Ex

Post: In

Ex

PSI: Pre: Total

Mid: Total

Post: Total 
Appendix B

Therapist Demographic Form 
Therapist Number

\section{Therapist Demographic Form}

Sex: $M / F$

Age

Ethnicity

Primary Language:

Secondary Language:

Highest level of education attained:

Total number of years as a clinician:

\section{$\underline{\text { PCIT Experience }}$}

Initial Training--

Online Training: Y / N 30 Hour Training: Y / N 40 Hour Training: Y / N

2 Day Advanced Training: Y / N

Years Practicing PCIT

Number of PCIT cases started (at baseline)

Number of PCIT cases graduated (at baseline) 
Appendix C

Session Record Form 
Therapist Number

\section{Session Record Form}

Participant Number:

Session Number:

Date:

Date of Last Session Attended:

Session Attendance (circle below)-

Arrived: On time

Late: minutes

Cancel

No-show

Homework Completion (check for both recording and sheet completion)

Video/Audio Recording- Days Completed:

Homework Sheet- Days Completed:

ECBI: Intensity

Problem

DPICS: (CDI Coded Interactions)

Total Positive Composite (Labeled Praises + Behavioral Descriptions + Reflections)

Total Negative Composite (Negative Talk + Questions + Commands)

Prize bags attained during session (mark):

Timely Attendance

$>50 \%$ Homework Completion (recording)

Mastery: CDI

PDI

Graduation 